Please do not remove this page

RMIT

UNIVERSITY

\title{
Acupuncture for treating fibromyalgia (Review)
}

Deare, John; Zheng, Zhen; Xue, Charlie Changli; Liu, Jianping; Shang, Jingsheng; Scott, Sean; Littlejohn, Geoff

https://researchrepository.rmit.edu.au/esploro/outputs/9921859987901341/filesAndLinks?institution=61RMIT_INST\&index=null

Deare, J., Zheng, Z., Xue, C. C., Liu, J., Shang, J., Scott, S., \& Littlejohn, G. (2013). Acupuncture for treating fibromyalgia (Review). Cochrane Database of Systematic Reviews, 5, 1-5.

https://doi.org/10.1002/14651858.CD007070.pub2

Published Version: https://doi.org/10.1002/14651858.CD007070.pub2

Repository homepage: https://researchrepository.rmit.edu.au

(c) 2013 The Cochrane Collaboration. Published by John Wiley and Sons, Ltd.

Downloaded On 2023/04/27 01:10:22 +1000 
Thank you for downloading this document from the RMIT Research Repository.

The RMIT Research Repository is an open access database showcasing the research outputs of RMIT University researchers.

RMIT Research Repository: http://researchbank.rmit.edu.au/

\section{Citation:}

Deare, J, Zheng, Z, Xue, C, Liu, J, Shang, J, Scott, S and Littlejohn, G 2013, 'Acupuncture for treating fibromyalgia', Cochrane Database of Systematic Reviews, no. 5, CD007070, pp. $1-5$.

See this record in the RMIT Research Repository at:

http://researchbank.rmit.edu.au/view/rmit:23283

Version: Published Version

Copyright Statement: (c) 2013 The Cochrane Collaboration. Published by John Wiley \& Sons, Ltd

Link to Published Version:

http://dx.doi.org/10.1002/14651858.CD007070.pub2 


\title{
Acupuncture for treating fibromyalgia (Review)
}

\author{
Deare JC, Zheng Z, Xue CCL, Liu JP, Shang J, Scott SW, Littlejohn G
}

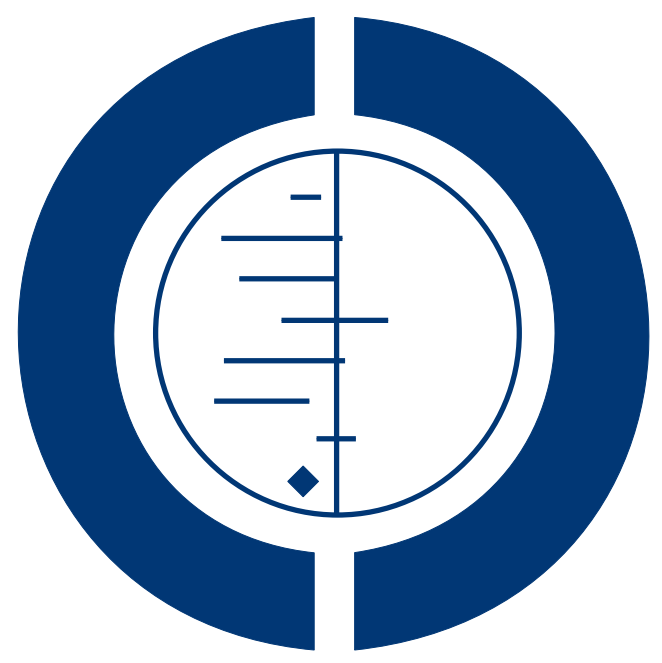

THE COCHRANE COLLABORATION $^{\circledR}$

This is a reprint of a Cochrane review, prepared and maintained by The Cochrane Collaboration and published in The Cochrane Library 2013, Issue 5

http://www.thecochranelibrary.com

\section{WILEY}


TABLE OF CONTENTS

HEADER . . . . . . . . . . . . . . . . . . . . . . . . . . . . . . . . . . . . . . . . . . . . . . . . . . . .

ABSTRACT . . . . . . . . . . . . . . . . . . . . . . . . . . . . . . . . . . . . . . . . . . . . . . . . . . .

PLAIN LANGUAGE SUMMARY . . . . . . . . . . . . . . . . . . . . . . . . . . . . . . . . . . . . . . . . 2

SUMMARY OF FINDINGS FOR THE MAIN COMPARISON $\quad . \quad$. . . . . . . . . . . . . . . . . . . . . . . . . . .

BACKGROUND . . . . . . . . . . . . . . . . . . . . . . . . . . . . . . . . . . . . . 8

OBJECTIVES . . . . . . . . . . . . . . . . . . . . . . . . . . . . . . . . . . . . . . . . . . . . . . . . . .

METHODS . . . . . . . . . . . . . . . . . . . . . . . . . . . . . . . . . . . . . . . . .

Figure 1. . . . . . . . . . . . . . . . . . . . . . . . . . . . . . . . . . . . . . . . . 11

RESULTS . . . . . . . . . . . . . . . . . . . . . . . . . . . . . . . . . . . . . . . . 14

Figure 2. . . . . . . . . . . . . . . . . . . . . . . . . . . . . . . . . . . . . . 18

Figure 3. . . . . . . . . . . . . . . . . . . . . . . . . . . . . . . . . . . . . . . 19

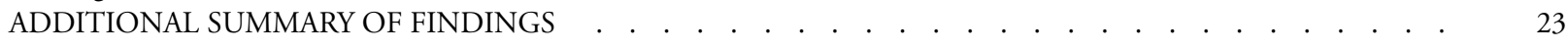

DISCUSSION . . . . . . . . . . . . . . . . . . . . . . . . . . . . . . . . . . . . . . . . . . . . .

AUTHORS' CONCLUSIONS . . . . . . . . . . . . . . . . . . . . . . . . . . . . . . . . . . . .

ACKNOWLEDGEMENTS . . . . . . . . . . . . . . . . . . . . . . . . . . . . . . . . . . . . . . .

REFERENCES . . . . . . . . . . . . . . . . . . . . . . . . . . . . . . . . . . . . . . . . 38

CHARACTERISTICS OF STUDIES . . . . . . . . . . . . . . . . . . . . . . . . . . . . . . . . . . . . . . . . . . .

DATA AND ANALYSES . . . . . . . . . . . . . . . . . . . . . . . . . . . . . . . . . . . . . . . . . . . 68

Analysis 1.1. Comparison 1 Acupuncture versus non-acupuncture treatment, Outcome 1 Pain up to 1 month after treatment.

Analysis 1.2. Comparison 1 Acupuncture versus non-acupuncture treatment, Outcome 2 Global well-being: rated by participants up to 1 month after treatment.

Analysis 1.3. Comparison 1 Acupuncture versus non-acupuncture treatment, Outcome 3 Sleep up to 1 month after treatment.

Analysis 1.4. Comparison 1 Acupuncture versus non-acupuncture treatment, Outcome 4 Fatigue up to 1 month after treatment.

Analysis 1.5. Comparison 1 Acupuncture versus non-acupuncture treatment, Outcome 5 Stiffness up to 1 month after treatment.

Analysis 1.6. Comparison 1 Acupuncture versus non-acupuncture treatment, Outcome 6 Adverse events.

Analysis 1.7. Comparison 1 Acupuncture versus non-acupuncture treatment, Outcome 7 Mental well-being up to 1 month

Analysis 2.1. Comparison 2 Acupuncture versus placebo or sham acupuncture, Outcome 1 Pain (subgroup EA \& MA) up to 1 month after treatment.

Analysis 2.2. Comparison 2 Acupuncture versus placebo or sham acupuncture, Outcome 2 Pain follow-up to 7 months after treatment (subgroup EA vs MA).

Analysis 2.3. Comparison 2 Acupuncture versus placebo or sham acupuncture, Outcome 3 Pain: sham non-invasive acupuncture (not breaking skin) vs sham invasive acupuncture (breaking skin). . . . . . . . . . . . . . .

Analysis 2.4. Comparison 2 Acupuncture versus placebo or sham acupuncture, Outcome 4 Physical function (SF-36).

Analysis 2.5. Comparison 2 Acupuncture versus placebo or sham acupuncture, Outcome 5 Global well-being: rated by participants (subgroup EA vs MA) up to 1 month after treatment. . . . . . . . . . . . . . . . . . . . . . .

Analysis 2.6. Comparison 2 Acupuncture versus placebo or sham acupuncture, Outcome 6 Global well-being follow-up to 7 months after treatment (subgroup EA \& MA).

Analysis 2.7. Comparison 2 Acupuncture versus placebo or sham acupuncture, Outcome 7 Sleep (subgroup EA \& MA) up to 1 month after treatment.

Analysis 2.8. Comparison 2 Acupuncture versus placebo or sham acupuncture, Outcome 8 Sleep follow-up to 7 months after treatment (subgroup EA \& MA).

Analysis 2.9. Comparison 2 Acupuncture versus placebo or sham acupuncture, Outcome 9 Fatigue (subgroup EA vs MA) up to 1 month after treatment. . . . . . . . . . . . . . . . . . . . . . . . . . . . . . . . . . . . . . .

Analysis 2.10. Comparison 2 Acupuncture versus placebo or sham acupuncture, Outcome 10 Fatigue follow-up to 7 months after treatment (subgroup EA vs MA). 
Analysis 2.11. Comparison 2 Acupuncture versus placebo or sham acupuncture, Outcome 11 Stiffness up to 1 month after treatment.

Analysis 2.12. Comparison 2 Acupuncture versus placebo or sham acupuncture, Outcome 12 Stiffness follow-up to 7 months after treatment.

Analysis 2.13. Comparison 2 Acupuncture versus placebo or sham acupuncture, Outcome 13 Adverse events.

Analysis 2.14. Comparison 2 Acupuncture versus placebo or sham acupuncture, Outcome 14 Mental well-being up to 1 month after treatment. . . . . . . . . . . . . . . . . . . . . . . . . . . . . . . . . . .

Analysis 2.15. Comparison 2 Acupuncture versus placebo or sham acupuncture, Outcome 15 Mental well-being follow-up to 7 months. . . . . . . . . . . . . . . . . . . . . . . . . . . . . . . . . . .

Analysis 2.16. Comparison 2 Acupuncture versus placebo or sham acupuncture, Outcome 16 Analgesic use (number of tablets per week) up to 1 month after treatment. . . . . . . . . . . . . . . . . . . . . . . . . . . . . . . .

Analysis 2.17. Comparison 2 Acupuncture versus placebo or sham acupuncture, Outcome 17 Analgesic use (number of participants taking analgesics up to 1 month after treatment).

Analysis 2.18. Comparison 2 Acupuncture versus placebo or sham acupuncture, Outcome 18 Tenderness up to 1 month after treatment.

Analysis 2.19. Comparison 2 Acupuncture versus placebo or sham acupuncture, Outcome 19 Overall well-being: rated by care giver at end of treatment.

Analysis 3.1. Comparison 3 Acupuncture versus me. . . . . . . . . . .

Analysis 3.2. Comparison 3 Acupuncture versus medication, Outcome 2 Adverse events.

Analysis 3.3. Comparison 3 Acupuncture versus medication, Outcome 3 Tenderness up to 1 month after treatment.

Analysis 4.1. Comparison 4 Acupuncture as an adjunct therapy, Outcome 1 Pain up to 1 month after treatment. . .

Analysis 4.2. Comparison 4 Acupuncture as an adjunct therapy, Outcome 2 Pain up to 7 months after treatment.

Analysis 4.3. Comparison 4 Acupuncture as an adjunct therapy, Outcome 3 Adverse events. . . . . . . . . . . .

Analysis 4.4. Comparison 4 Acupuncture as an adjunct therapy, Outcome 4 Tenderness - number of tender points below $\mathrm{kg} / \mathrm{cm}^{2}$ up to 1 month after treatment.

Analysis 4.5. Comparison 4 Acupuncture as an adjunct therapy, Outcome 5 Tenderness - number of tender points below $\mathrm{kg} / \mathrm{cm}^{2}$ up to 7 month after treatment.

Analysis 4.6. Comparison 4 Acupuncture as an adjunct therapy, Outcome 6 Tenderness - mean pressure threshold by pressure algometry up to 1 month after treatment.

Analysis 4.7. Comparison 4 Acupuncture as an adjunct therapy, Outcome 7 Tenderness - mean pressure threshold by pressure algometry, follow up to 7 months after treatment.

Analysis 5.1. Comparison 5 Deep needling with stimulation (T/S) versus deep needling without stimulation (T/O), Outcome 1 Pain up to 1 month after treatment. . . . . . . . . . . . . . . . . . . . . . . . . . . . . . . .

Analysis 5.2. Comparison 5 Deep needling with stimulation (T/S) versus deep needling without stimulation (T/O), Outcome 2 Physical function (SF-36) up to 1 month after treatment.

Analysis 5.3. Comparison 5 Deep needling with stimulation (T/S) versus deep needling without stimulation (T/O), Outcome 3 Fatigue up to 1 month after treatment. . . . . . . . . . . . . . . . . . . . . . . . . .

APPENDICES . . . . . . . . . . . . . . . . . . . . . . . . . . . . . . . . . . . . . . . . . . . . . .

WHAT'S NEW . . . . . . . . . . . . . . . . . . . . . . . . . . . . . . . . . . . . . . . . . . . .

CONTRIBUTIONS OF AUTHORS . . . . . . . . . . . . . . . . . . . . . . . . . . . . . . . . . . . .

DECLARATIONS OF INTEREST . . . . . . . . . . . . . . . . . . . . . . . . . . . . . . . . . . . . . . . . . . .

SOURCES OF SUPPORT . . . . . . . . . . . . . . . . . . . . . . . . . . . . . . . . . 


\title{
[Intervention Review]
}

\section{Acupuncture for treating fibromyalgia}

\author{
John C Deare ${ }^{1}$, Zhen Zheng ${ }^{2}$, Charlie CL Xue ${ }^{2}$, Jian Ping Liu ${ }^{3}$, Jingsheng Shang ${ }^{4}$, Sean W Scott ${ }^{5}$, Geoff Littlejohn ${ }^{6}$ \\ ${ }^{1}$ Compmed Health Institute, Southport, Queensland, Australia; and Traditional \& Complementary Medicine Program, Health Innova- \\ tions Research Institute, Discipline of Chinese Medicine, School of Health Sciences, RMIT University, Bundoora, Australia. ${ }^{2}$ Traditional \\ \& Complementary Medicine Research Program, Health Innovations Research Institute and Discipline of Chinese Medicine, School of \\ Health Sciences, RMIT University, Bundoora, Australia. ${ }^{3}$ Centre for Evidence-Based Chinese Medicine, Beijing University of Chinese \\ Medicine, Beijing, China. ${ }^{4}$ Akupunktoren Bruno Aamo AS, Tromsoe, Norway. ${ }^{5}$ Department of Emergency Medicine, Royal North \\ Shore Hospital, St Leonards, Australia. ${ }^{6}$ Department of Rheumatology, Monash Medical Centre, Clayton, Australia
}

Contact address: Zhen Zheng, Traditional \& Complementary Medicine Research Program, Health Innovations Research Institute and Discipline of Chinese Medicine, School of Health Sciences, RMIT University, PO Box 71, Bundoora, Victoria, 3083, Australia. zhen.zheng@rmit.edu.au.

Editorial group: Cochrane Musculoskeletal Group.

Publication status and date: New, published in Issue 5, 2013.

Review content assessed as up-to-date: 15 January 2012.

Citation: Deare JC, Zheng Z, Xue CCL, Liu JP, Shang J, Scott SW, Littlejohn G. Acupuncture for treating fibromyalgia. Cochrane Database of Systematic Reviews 2013, Issue 5. Art. No.: CD007070. DOI: 10.1002/14651858.CD007070.pub2.

Copyright (C) 2013 The Cochrane Collaboration. Published by John Wiley \& Sons, Ltd.

\begin{abstract}
A B S T R A C T

\section{Background}

One in five fibromyalgia sufferers use acupuncture treatment within two years of diagnosis.
\end{abstract}

\section{Objectives}

To examine the benefits and safety of acupuncture treatment for fibromyalgia.

\section{Search methods}

We searched CENTRAL, PubMed, EMBASE, CINAHL, National Research Register, HSR Project and Current Contents, as well as the Chinese databases VIP and Wangfang to January 2012 with no language restrictions.

\section{Selection criteria}

Randomised and quasi-randomised studies evaluating any type of invasive acupuncture for fibromyalgia diagnosed according to the American College of Rheumatology (ACR) criteria, and reporting any main outcome: pain, physical function, fatigue, sleep, total wellbeing, stiffness and adverse events.

\section{Data collection and analysis}

Two author pairs selected trials, extracted data and assessed risk of bias. Treatment effects were reported as standardised mean differences (SMD) and 95\% confidence intervals (CI) for continuous outcomes using different measurement tools (pain, physical function, fatigue, sleep, total well-being and stiffness) and risk ratio (RR) and 95\% CI for dichotomous outcomes (adverse events). We pooled data using the random-effects model.

Acupuncture for treating fibromyalgia (Review)

Copyright @ 2013 The Cochrane Collaboration. Published by John Wiley \& Sons, Ltd. 


\section{Main results}

Nine trials (395 participants) were included. All studies except one were at low risk of selection bias; five were at risk of selective reporting bias (favouring either treatment group); two were subject to attrition bias (favouring acupuncture); three were subject to performance bias (favouring acupuncture) and one to detection bias (favouring acupuncture). Three studies utilised electro-acupuncture (EA) with the remainder using manual acupuncture (MA) without electrical stimulation. All studies used 'formula acupuncture' except for one, which used trigger points.

Low quality evidence from one study (13 participants) showed EA improved symptoms with no adverse events at one month following treatment. Mean pain in the non-treatment control group was 70 points on a 100 point scale; EA reduced pain by a mean of 22 points (95\% confidence interval (CI) 4 to 41), or $22 \%$ absolute improvement. Control group global well-being was 66.5 points on a 100 point scale; EA improved well-being by a mean of 15 points (95\% CI 5 to 26 points). Control group stiffness was 4.8 points on a 0 to 10 point; EA reduced stiffness by a mean of 0.9 points (95\% CI 0.1 to 2 points; absolute reduction $9 \%$, 95\% CI $4 \%$ to $16 \%$ ). Fatigue was 4.5 points ( 10 point scale) without treatment; EA reduced fatigue by a mean of 1 point ( $95 \%$ CI 0.22 to 2 points), absolute reduction $11 \%$ ( $2 \%$ to $20 \%$ ). There was no difference in sleep quality (MD 0.4 points, $95 \%$ CI -1 to 0.21 points, 10 point scale), and physical function was not reported.

Moderate quality evidence from six studies (286 participants) indicated that acupuncture (EA or MA) was no better than sham acupuncture, except for less stiffness at one month. Subgroup analysis of two studies (104 participants) indicated benefits of EA. Mean pain was 70 points on 0 to 100 point scale with sham treatment; EA reduced pain by $13 \%$ (5\% to 22\%); (SMD -0.63, 95\% CI 1.02 to -0.23 ). Global well-being was 5.2 points on a 10 point scale with sham treatment; EA improved well-being: SMD 0.65 , $95 \%$ CI 0.26 to 1.05 ; absolute improvement $11 \%$ (4\% to $17 \%$ ). EA improved sleep, from 3 points on a 0 to 10 point scale in the sham group: SMD 0.40 (95\% CI 0.01 to 0.79$)$; absolute improvement $8 \%$ ( $0.2 \%$ to $16 \%)$. Low-quality evidence from one study suggested that MA group resulted in poorer physical function: mean function in the sham group was 28 points (100 point scale); treatment worsened function by a mean of 6 points (95\% CI -10.9 to -0.7). Low-quality evidence from three trials (289 participants) suggested no difference in adverse events between real (9\%) and sham acupuncture (35\%); RR 0.44 (95\% CI 0.12 to 1.63 ).

Moderate quality evidence from one study (58 participants) found that compared with standard therapy alone (antidepressants and exercise), adjunct acupuncture therapy reduced pain at one month after treatment: mean pain was 8 points on a 0 to 10 point scale in the standard therapy group; treatment reduced pain by 3 points ( $95 \%$ CI -3.9 to -2.1 ), an absolute reduction of $30 \%$ ( $21 \%$ to $39 \%$ ). Two people treated with acupuncture reported adverse events; there were none in the control group (RR 3.57; $95 \%$ CI 0.18 to 71.21 ). Global well-being, sleep, fatigue and stiffness were not reported. Physical function data were not usable.

Low quality evidence from one study (38 participants) showed a short-term benefit of acupuncture over antidepressants in pain relief: mean pain was 29 points ( 0 to 100 point scale) in the antidepressant group; acupuncture reduced pain by 17 points ( $95 \%$ CI -24.1 to -10.5). Other outcomes or adverse events were not reported.

Moderate-quality evidence from one study (41 participants) indicated that deep needling with or without deqi did not differ in pain, fatigue, function or adverse events. Other outcomes were not reported.

Four studies reported no differences between acupuncture and control or other treatments described at six to seven months follow-up.

No serious adverse events were reported, but there were insufficient adverse events to be certain of the risks.

\section{Authors' conclusions}

There is low to moderate-level evidence that compared with no treatment and standard therapy, acupuncture improves pain and stiffness in people with fibromyalgia. There is moderate-level evidence that the effect of acupuncture does not differ from sham acupuncture in reducing pain or fatigue, or improving sleep or global well-being. EA is probably better than MA for pain and stiffness reduction and improvement of global well-being, sleep and fatigue. The effect lasts up to one month, but is not maintained at six months followup. MA probably does not improve pain or physical functioning. Acupuncture appears safe. People with fibromyalgia may consider using EA alone or with exercise and medication. The small sample size, scarcity of studies for each comparison, lack of an ideal sham acupuncture weaken the level of evidence and its clinical implications. Larger studies are warranted.

\section{PLAIN LANGUAGE SUMMARY}

\section{Acupuncture for fibromyalgia}


This summary of a Cochrane review presents what we know from research about the effect of acupuncture on fibromyalgia.

\section{The review shows that in people with fibromyalgia:}

- acupuncture is probably better than non-acupuncture treatment in reducing pain and stiffness and improving overall well-being and fatigue;

- acupuncture with electrical stimulation is probably better than needling alone in reducing pain and stiffness, and improving overall well-being, sleep and fatigue;

- acupuncture without electrical stimulation probably does not reduce pain or improve fatigue, overall well-being or sleep; and

- acupuncture probably enhances the effect of drugs and exercise on pain.

\section{What is fibromyalgia and what is acupuncture?}

When you have fibromyalgia, you experience pain in many sites of your body, with a range of other symptoms including joint stiffness, sleep disturbance, fatigue and mood disorders, which affect the quality of life. There is no cure and few treatment options for fibromyalgia at present, so the treatments aim to relieve pain and improve your well-being and the ability to function.

Acupuncture is a form of Chinese medicine and uses fine needles to stimulate certain areas of the body, called acupuncture points. Acupuncture is commonly used by people to reduce various forms of pain. It works by reducing inflammation, stimulating the release of your body's own pain killer, that is endorphins, and calming your brain. It is safe with few, short-lasting side effects. If supported by the overall body of evidence, acupuncture will offer much needed effective symptom relief for fibromyalgia.

\section{Best estimate of what happens to people with fibromyalgia who use acupuncture:}

\section{Comparing acupuncture with sham interventions}

Pain (higher scores mean worse or more severe pain)

- People who had needle acupuncture with electrical stimulation rated their pain to be 13 points lower on a 100-point scale (absolute improvement) after six sessions of treatment.

- People who had fake acupuncture rated their pain to be 70 on a scale of 0 to 100 at the end of treatment.

- People who had needle acupuncture with electrical stimulation rated their pain to be 57.

Physical function (higher scores mean better function):

- People who used needle acupuncture without electrical stimulation rated their physical function to be six points lower (absolute deterioration).

- People who had fake treatment rated their physical function to be 28 on a scale of 0 to 100 at the end of treatment.

- People who had needle acupuncture without electrical stimulation rated their physical function to be 22 .

- There are no data on needle acupuncture with electrical stimulation.

Global well-being rated by participants (higher scores mean better function):

- People who had needle acupuncture with electrical stimulation rated their well-being to be 11 points higher (absolute improvement).

- People who had fake treatment rated their well-being to be 41 on a scale of 0 to 100 at the end of treatment.

- People who had needle acupuncture with electrical stimulation rated their well-being to be 52 .

Sleep (higher scores mean better sleep):

- People who used acupuncture rated their sleep to be eight points higher (absolute improvement).

- People who had fake treatment rated their sleep to be 30 on a scale of 0 to 100 at the end of treatment.

- People who had needle acupuncture with electrical stimulation rated their sleep to be 38 .

Acupuncture for treating fibromyalgia (Review)

Copyright $\odot 2013$ The Cochrane Collaboration. Published by John Wiley \& Sons, Ltd. 
Fatigue (higher scores mean more severe fatigue):

- People who had needle acupuncture with electrical stimulation rated their fatigue to be 15 points lower (absolute improvement).

- People who had fake treatment rated their fatigue to be 78 on a scale of 0 to 100 .

- People who had needle acupuncture with electrical stimulation rated their fatigue to be 63 .

Stiffness (higher scores mean more severe stiffness):

- People who had needle acupuncture with electrical stimulation rated their stiffness to be nine points lower (absolute improvement).

- People who had fake treatment rated their stiffness to be 66 on a scale of 0 to 100 at the end of treatment.

- People who had needle acupuncture with electrical stimulation rated their stiffness to be 57.

- Data on needle acupuncture without electrical acupuncture were not available.

Adverse effects:

- One in six people who had acupuncture reported adverse events.

- One in three people who had fake treatments reported adverse events.

- Overall, such events were minor and lasted less than one day.

\section{Acupuncture as an adjunct therapy}

Pain (higher scores mean more severe pain):

- People who had needle acupuncture in addition to a standard treatment of exercise and medication (antidepressants) rated their pain to be 30 points lower on a scale of 0 to 100 (absolute improvement) after 20 sessions of acupuncture.

- People who had standard therapy rated their pain to be 80 on a scale of 0 to 100 at the end of treatment.

- People who had additional acupuncture treatment rated their pain to be 50 .

\section{Acupuncture compared with antidepressants}

Pain (higher scores mean more severe pain):

- People who had acupuncture rated their pain to be 17 points lower (absolute improvement) after 28 sessions of acupuncture.

- People who had antidepressants rated their pain to be 29 on a scale of 0 to 100 at the end of treatment.

- People who had acupuncture treatment rated their pain to be 12 .

\section{Comparing acupuncture with non-acupuncture (wait list)}

- People who had needle acupuncture with electrical stimulation rated 23, 11 and 9 points lower on a 100-point scale for pain, fatigue and stiffness, respectively; and reported their global well-being to be 15 points better than those who did not have acupuncture. 


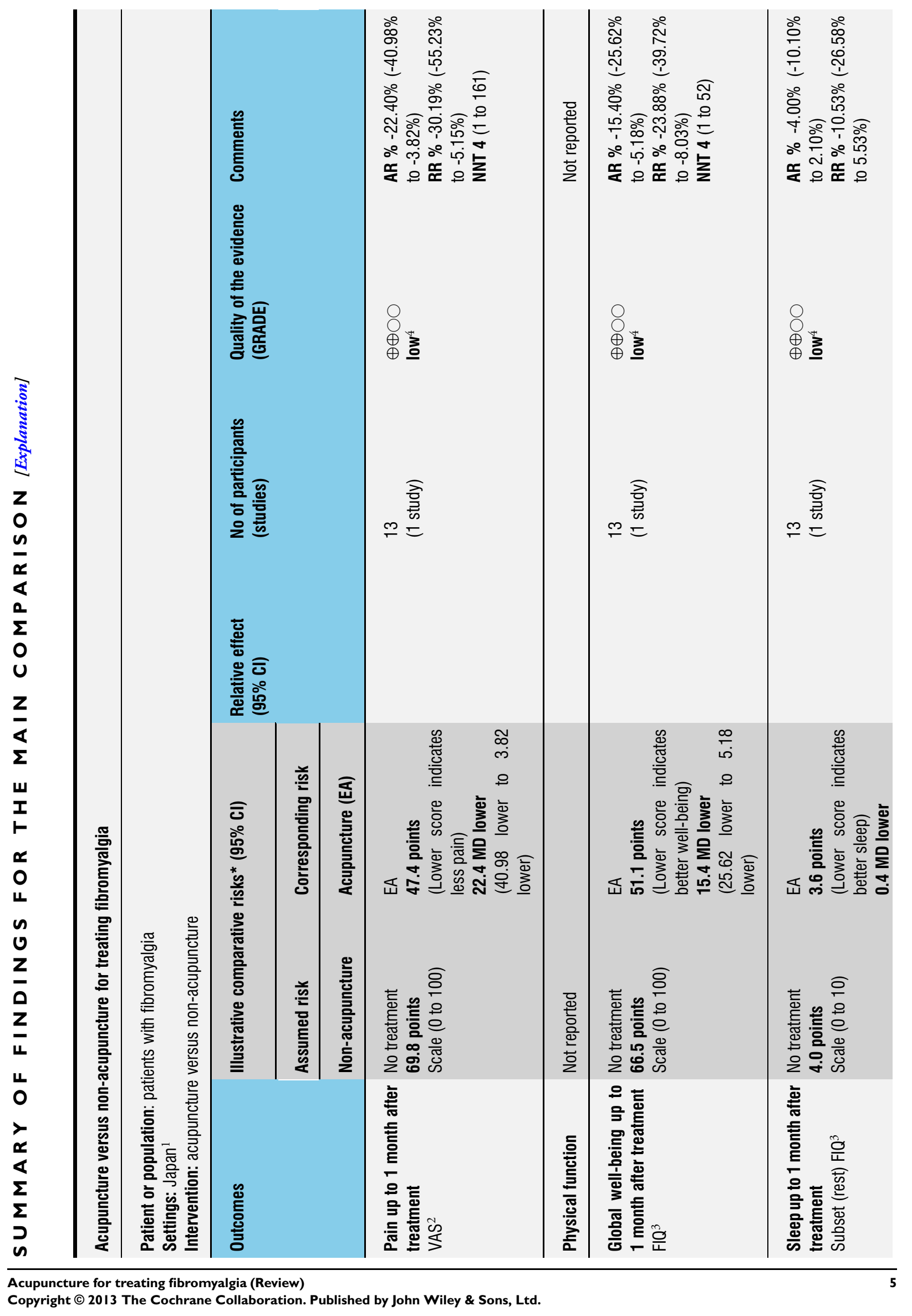




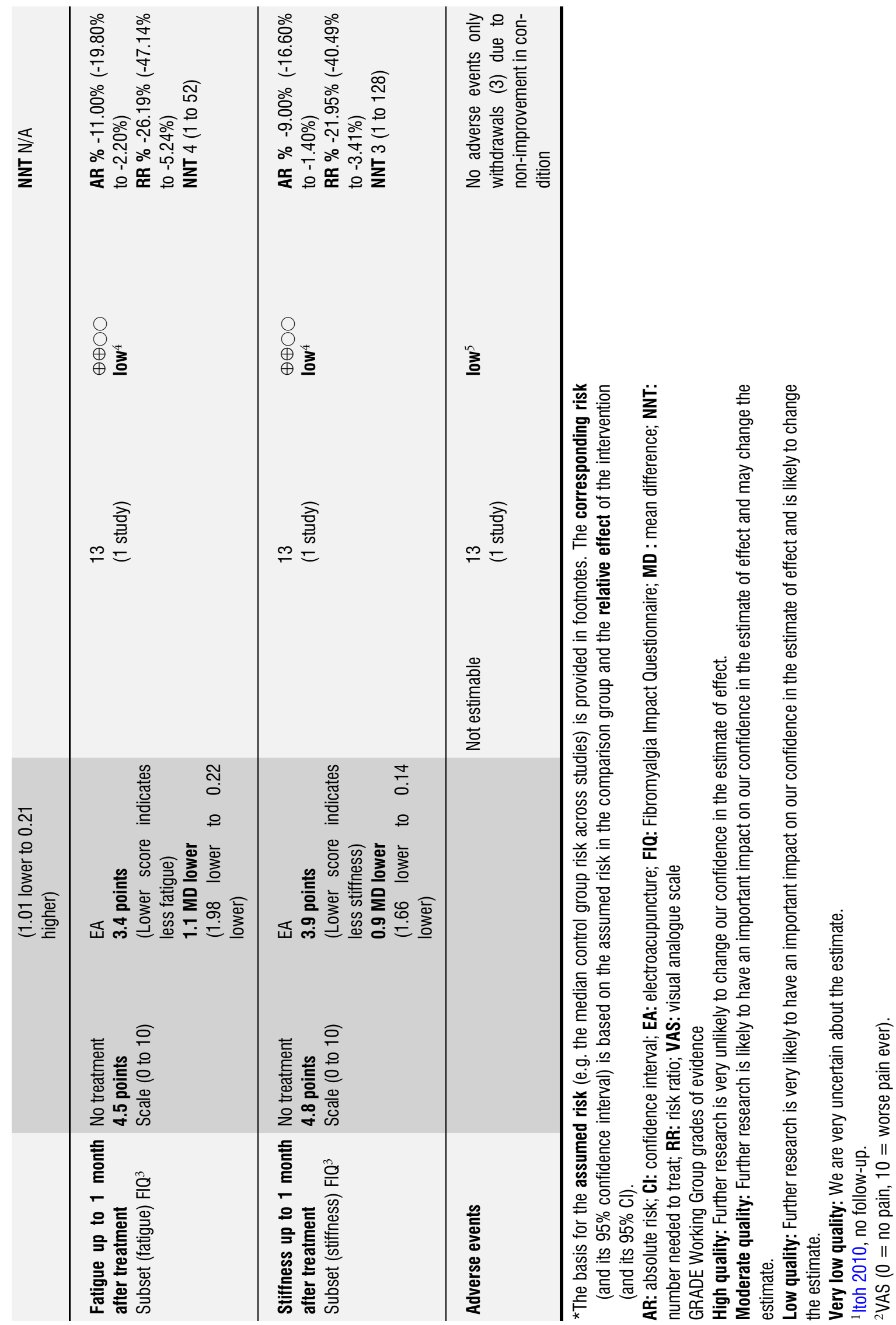




$$
L
$$




\section{B A C K G R O U N D}

Fibromyalgia is a musculoskeletal disorder characterised by widespread chronic pain and any number of co-morbidities, such as sleep disturbance, fatigue, stiffness, irritable bowel syndrome, headaches and mood disorders. It affects over $2 \%$ of the population and occurs predominantly in females (Wallace 2005). There was, until recently, no pharmacotherapy that effectively addressed all the symptoms associated with fibromyalgia (Lawson 2006). The United States of America's Food and Drug Administration (FDA) has approved 'Lyrica' (pregabalin), 'Savella' (milnacipran $\mathrm{HCl}$ ) and 'Cymbalta' (duloxetine hydrochloride) (Boomershine 2009) for the treatment of fibromyalgia. In contrast, to date the European Medicines Agency has not approved any pharmacotherapy for the treatment of fibromyalgia (www.fibroaction.org), suggesting that the approved FDA drugs for fibromyalgia are not readily accessible by people globally. Non-drug therapies, such as cognitive behavioural therapy (CBT) and exercise, or a combination of the two approaches, are potentially beneficial for people with fibromyalgia (Nüesch 2012).

With respect to complementary and alternative medicine (CAM), acupuncture, a physical therapy of Traditional Chinese Medicine (TCM) that has been used to treat chronic pain for over two millennia in China, is promising for alleviating the symptoms associated with fibromyalgia (Bergman 2007). Among fibromyalgia sufferers, 91\% had used CAM (Pioro-Boisset 1996) and one in five sufferers had sought acupuncture for treatment within two years of diagnosis (Bombardier 1996). In 1998, the National Institutes of Health Consensus Development Conference on Acupuncture stated that acupuncture may be used as an adjunct therapy for fibromyalgia (NIH 1998).

However, no therapy alone has been demonstrated to be universally superior to the others. Consequently, it was considered appropriate that when treating fibromyalgia a multidisciplinary approach be used (Arnold 2006). In the United States of America, approximately one million consumers use acupuncture annually (Burke 2006; Ezzo 2000). Furthermore, acupuncture is a relatively safe intervention (Vincent 2001) when compared with pharmacotherapies. Adverse events associated with acupuncture tend to be mild and short-lasting, such as lethargy and pain at the needling sites (MacPherson 2004).

The plausible mechanism of acupuncture analgesia is its effect on the central nervous system and consequent regulation of neurotransmitters and hormones. Acupuncture stimulates nerve fibres (e.g. A delta afferents), which in turn activate transmission neurons in the dorsal laminae of the spinal cord and further activate three levels of the endogenous pain modulation systems at the spinal cord, midbrain, thalamus and hypothalamus. The activation results in a cascade of pain-modulating endorphins, serotonin and noradrenaline, which contributes to analgesia (Cao 2002; Han 1997; Sims 1997).
Although the pathophysiology of fibromyalgia is not well understood, data suggest that ineffective descending inhibition of the central nervous system may cause an abnormal modulation of sensory inputs (such as mechanoreceptor inputs), resulting in pain (Price 2005). Acupuncture action enhances the function of the endogenous pain inhibition systems and therefore may be beneficial to people with fibromyalgia.

The World Health Organization (WHO) defines real acupuncture, in its broadest sense, as the insertion of needles into the human body surface for therapeutic purposes (WHO 2007). Throughout its history, different treatment styles of acupuncture have been developed in relation to needle size, depth of needling and duration of needle retention as well as deqi sensation. Deqi is the feeling of soreness, numbness, distension, heaviness or the electric shock sensation that occurs around a correctly placed and manipulated acupuncture needle (WHO 2007).

A number of different styles of acupuncture are presently in use, according to acupuncture point selection and stimulation modes. In clinical practice, the selection of acupuncture points for each patient is based on either a Chinese medicine diagnosis (individualised acupuncture treatment) or symptom alleviation (formula acupuncture treatment). Sometimes trigger points are also selected for needling and this may be described as dry needling. There is also micro-system acupuncture where needles are mainly inserted into defined points on an anatomical part of the body such as the head (scalp acupuncture), the ear (auricular acupuncture) or the hand (hand acupuncture). Needles can be deeply inserted into soft tissue and manipulated to elicit deqi (also known as traditional Chinese acupuncture) or superficially inserted into the skin without eliciting deqi (which may be described as Japanese acupuncture/meridian therapy).

Apart from, and in addition to, needles, acupuncture points or other points mentioned above can be stimulated using heat (such as moxibustion), with electrical current (known as electro-acupuncture), using mechanical pressure (acupressure) or using laser (laser acupuncture). Of all the forms of stimulation of acupuncture points, needling involving skin penetration (manual acupuncture) is the most commonly used method.

In 2007, a systematic review of acupuncture for fibromyalgia concluded that "acupuncture could not be recommended for fibromyalgia" (Mayhew 2007). However, it appears that this review neither searched for nor included studies from Chinese databases. In addition, new studies have been published. Therefore, there is a need to perform a thorough review to allow an up-to-date assessment of all available studies to determine the potential role of acupuncture in the management of fibromyalgia.

\section{O B JE C T IVES}


The present review aims to determine whether real acupuncture is more beneficial in terms of pain reduction, function and wellbeing improvement than placebo and other treatments and is safe in people with fibromyalgia. We examined the following comparisons:

\section{Acupuncture versus no acupuncture (e.g. wait list) \\ 2. Acupuncture versus placebo or sham acupuncture \\ 3. Acupuncture versus standard/usual care (e.g. cognitive behavioural therapy (CBT) and/or exercise and/or pharmacotherapy)}

4. Acupuncture as an adjunct therapy to standard/usual care (evaluating additional effect)

5. A particular style of acupuncture versus another (e.g. deep needling with stimulation versus deep needling without stimulation)

\section{METHODS}

\section{Criteria for considering studies for this review}

\section{Types of studies}

We considered randomised and quasi-randomised controlled clinical studies of acupuncture for treating patients with fibromyalgia. Quasi-randomised studies are those that do not strictly adhere to methods of true randomisation, e.g. location by the order of admission or date of birth. Inclusion of studies was not restricted according to language, type of publication or presence of blinding. We excluded studies from which we could not extract reported clinical outcomes or data for analyses.

\section{Types of participants}

Criteria for inclusion were participants of either gender, aged 18 and over, with a diagnosis of fibromyalgia according to the American College of Rheumatology (ACR) classification criteria for fibromyalgia (Wolfe 1990).

\section{Types of interventions}

Types of intervention were restricted to acupuncture that breaks the skin for therapeutic benefit (WHO 2002). Studies comparing different styles of acupuncture were also included. In addition, studies in which acupuncture was an adjunct therapy to other therapies (e.g. herbs, cupping, physiotherapy, exercise) were included, provided the control groups also received these therapies. Studies in which acupuncture points were stimulated with methods that did not break the skin, such as transcutaneous electrical nerve stimulation (TENS), infrared light, laser or digital pressure, were excluded. Acupuncture points refer to those points as defined in the Standard Acupuncture Nomenclature by the World Health Organization (WHO) (WHO 2002). Studies that used ashi acupuncture points (i.e. tender points) or trigger points were also to be included.

The control interventions included sham/fake/placebo acupuncture, other types of placebo control, non-acupuncture treatment, different styles of acupuncture or other treatment. An example of non-acupuncture treatment is a wait list. We considered standard care to be pharmacotherapy and/or exercise and/or CBT. When selecting studies that used sham/placebo acupuncture, we chose controls that did not intend to be an effective intervention, for example, needling on irrelevant acupuncture points, superficial needling or both. Other sham controls could have a disconnected electro-acupuncture stimulator, an inactive laser, mock TENS, infrared light or digital pressure. If there were sufficient studies, we planned to examine the differences between the various types of sham acupuncture (e.g. insertion verses non-insertion, deep needle verses shallow needle, on the acupuncture point versus off the acupuncture point). We excluded studies that did not provide adequate details of the control intervention.

\section{Types of outcome measures}

Included studies must have reported one or more of the following main clinical outcome measures related to pain, function and quality of life.

\section{Main outcomes}

1. Pain (e.g. visual analogue scale (VAS), numerical pain rating scale (NRS), McGill Pain Questionnaire (SF-MPQ), Multidimensional Pain Inventory (MPI) or Regional Pain Scale score)

2. Physical function (e.g. 36-Item Short-Form Health Survey (SF-36, Physical) or Health Assessment Questionnaire (HAQ))

3. Global well-being as rated by participants (e.g. Fibromyalgia Impact Questionnaire (FIQ), VAS rated by participants)

4. Sleep (e.g. VAS of intensity, numerical sleep scale 1 to 10)

5. Fatigue (e.g. VAS, Multidimensional Fatigue Inventory (MFI))

6. Morning stiffness (e.g. numerical scale)

7. Adverse events: proportion of participants who experienced an adverse event and proportion who withdrew due to adverse events

Provided the studies had main outcomes, we also considered any of the following minor outcomes.

\section{Minor outcomes}

1. Tenderness (e.g. number of tender points or pain threshold of tender points as measured with a dolorimeter) 
2. Mental well-being (e.g. SF-36 (mental), Hamilton Depression Rating Scale (HAMD))

3. Analgesic use (e.g. diary)

4. Changes in fibromyalgia symptoms (e.g. observer-rated change in fibromyalgia symptoms (including that rated by physicians))

5. Overall well-being rated by the study care givers

In the 'Summary of findings' table, we included the main outcomes of pain, physical function, global well-being, sleep, fatigue, stiffness and total adverse events (Arnold 2011).

\section{Search methods for identification of studies}

We initially searched the following databases from their inception to April 2008 as per protocol. We updated the search in May 2010 and January 2012. Search terms used included 'fibromyalgia' and 'acupuncture' and their variations (Figure 1). 
Figure I. A flow chart of study selection. ('English' refers to English databases and 'Chinese' refers to Chinese databases).

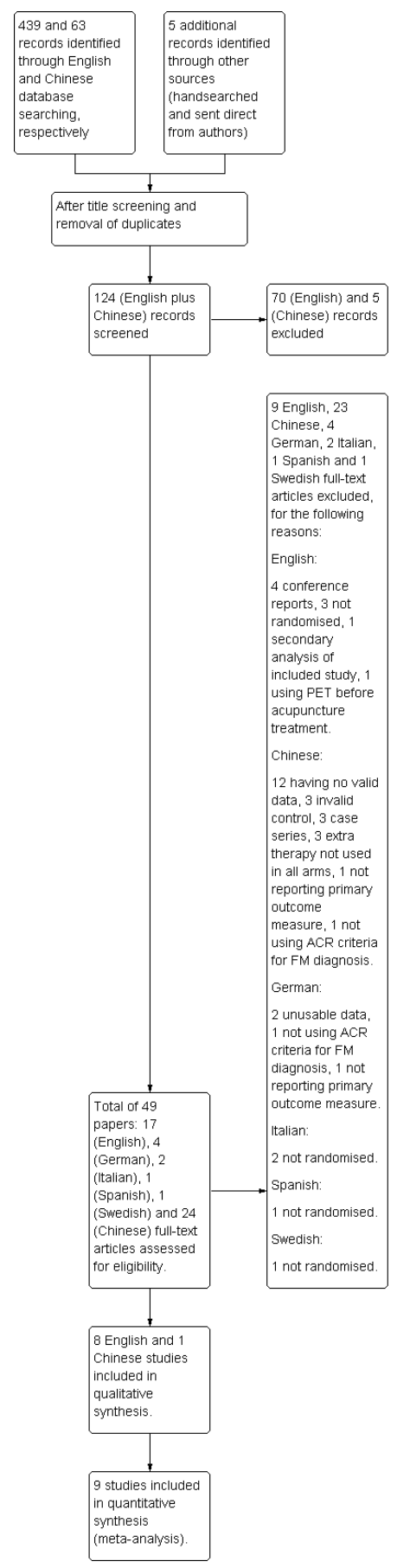


- Cochrane Central Register of Controlled studies (CENTRAL), via The Cochrane Library, Issue 1, 2012 ( www.thecochranelibrary.org) (Appendix 1)

- MEDLINE via PubMed, CAM PubMed and PubMed

Central (http://www.ncbi.nlm.nih.gov/pubmed/) (Appendix 2)

- EMBASE (http://ovidsp.tx.ovid.com/) (Appendix 3)

- CINAHL (http://www.ebscohost.com/) (Appendix 4)

- Chinese databases: Chongqing Weipu (VIP) (http://

lib.cqvip.com/) (Appendix 5) and Wanfang Database (http:// www.wanfangdata.com.cn/) (Appendix 6)

- Unpublished databases: National Research Register via the Department of Health, UK (www.dh.gov.uk) (Appendix 7); HSRProj via the National Library of Medicine, USA (http:// wwwcf.nlm.nih.gov) (Appendix 8)

- Current Contents (http:// apps.webofknowledge.com.ezproxy.lib.rmit.edu.au/) (Appendix 9)

\section{Additional studies}

We handsearched the bibliographies of review articles, excluded studies and textbooks on acupuncture, pain and fibromyalgia for additional studies. We contacted authors of published studies in an attempt to locate any unpublished studies.

\section{Data collection and analysis}

\section{Selection of studies}

One author (JD) searched the English language databases, while another author (ZZ) searched the Chinese language databases. These two authors independently examined the abstracts of the potential studies and obtained the full-text articles for consideration based on our pre-defined inclusion and exclusion criteria. We checked all references in the retrieved full-text English, Chinese and foreign language studies. Three potential papers were translated by the Cochrane Centres in Germany (one) and Italy (two). Three native speakers of Russian, Dutch and Spanish examined one paper each in these languages for potential studies. Four authors (JD, SS, ZZ, CX) with two in each group examined the English and Chinese studies, respectively, for inclusion/exclusion. Two authors (JD and ZZ) assessed the Harris 2008, Itoh 2010 and Targino 2008 papers. There were no disagreements between each pair of review authors.

\section{Data extraction}

Two author pairs (English: JD, SS and Chinese: ZZ, JSS) independently extracted data for each included study using our standard data extraction sheet. JD and ZZ extracted the data from Harris
2008, Itoh 2010 and Targino 2008. Data extracted included study characteristics, items related to the 'Risk of bias' tool and adverse events for each arm of the studies. We also extracted effect measures from each trial, including mean and standard deviation for continuous outcomes at or within one month of the end of the treatment; and number of events and number of participants in each group for dichotomous outcomes at the end of the treatment.

\section{Assessment of risk of bias in included studies}

Two review author groups, with two authors in each (JD, SS and ZZ, JSS), individually assessed the methodological quality of the English and Chinese studies, respectively, and incorporated them into the 'Risk of bias' tables. Items included in the tables are adequate sequence generation, allocation concealment (selection bias), blinding of the participants (performance bias), blinding of the assessor (detection bias), incomplete outcome data and its impact on the effect of estimate (attrition bias), and selective reporting (reporting bias). Using the extracted information, two authors (JD, ZZ) assessed whether they met the guidelines by selecting one of three choices: 'Yes', 'No' or 'Unclear' and reported the details of each decision in the allocated section of the table.

\section{Assessment of the quality of the acupuncture treatments}

To assess the quality of the acupuncture treatments, the two review author groups, all experienced clinical acupuncturists (minimum 10 years of experience each), used three instruments. We used the Standards for Reporting Interventions in Controlled Trials of Acupuncture (STRICTA) (MacPherson 2002) to extract the details of acupuncture intervention (Appendix 10), including acupuncture rationale, needling details, treatment regimen, co-interventions, practitioner background and control interventions, which are not addressed by other assessment tools. The purpose of STRICTA is to improve the reporting of interventions of controlled studies in acupuncture. This allows replication of the acupuncture treatment in other studies and clinical practice. As STRICTA does not offer a rating or scale to make a critical evaluation of the reporting, we further developed two rating systems to assess the adequacy of acupuncture treatment and confidence in the acupuncture diagnosis and treatment based on STRICTA data. Similar approaches have been used in other systematic reviews (Linde 2009; Scott 2006).

\section{Adequacy of acupuncture treatment protocol}

The rationale for examining the adequacy of treatment was to ensure that the study treatment protocol was comparable to routine 
clinical practice and the style of treatment was consistent with the techniques applied. For instance, a study claiming to be based on Chinese medicine but not eliciting deqi or only using a single needle would be considered inappropriate. Likewise, a treatment using appropriate Chinese medicine point selection but with only a single treatment would also be viewed as inadequate.

Assessment is based the on the following parts of the STRICTA table (Appendix 10).

- Acupuncture style

- Rationale for treatment/points used

- Literature sources

- Uni/bilateral

- Number of needles inserted

- Depth of insertion

- Response elicited

- Type of needle stimulation (electro/manual with or without tonification/dispersion etc.)

- Needle retention time

- Number of treatment sessions

- Frequency of treatments

From the list above, the review authors were required to judge if the acupuncture treatment performed was suitable for the style of acupuncture stated in the rationale for treatment. The experienced acupuncturists (JD, ZZ, JSS, SS) on the team rated the studies as low, medium or high according to whether the acupuncture treatment protocol was adequate. If there was insufficient information, we marked the study as 'insufficient information'.

\section{Confidence in the diagnosis and treatment delivery}

The determination of confidence in the administration of the acupuncture treatments was based on whether the person making the diagnosis, delivering the treatment or both was trained to the industry standard in that style. For example, L.Ac (licensed acupuncturist) would indicate meeting the USA standard. We used information about practitioners' training and practice background from STRICTA (Appendix 10) and information about the trial procedure to assess the level of confidence. For instance, it would be inappropriate to have an acupuncturist trained in Japanese/ meridian style, i.e. shallow needling on acupuncture points, to provide Chinese acupuncture. Equally, we did not consider it appropriate that acupuncturists who had no Chinese medicine differential diagnosis training to deliver Chinese medicine diagnosis and treatment, unless a well-explained protocol was in place or pre-trial training was given and competence of the trial acupuncturists was assessed prior to the commencement of the study. The review authors rated their confidence at three levels: low, medium or high.

\section{Measures of treatment effect}

We analysed the data according to the Cochrane guidelines. To examine the immediate effect, we used completed data at up to one month after the end of the treatment. This method has been used in other reviews (Vickers 2012). To examine the long-term effect, we extracted data collected up to seven months after the end of the treatment. We plotted outcomes from each study as point estimates with corresponding 95\% confidence intervals (CI) expressed as mean differences (MD) for continuous outcomes using the same scale, such as a 0 to 100 VAS for the measurement of pain or standardised mean differences (SMD) for continuous outcomes that used different scales, such as VAS and NRS for pain. We reported the number of adverse events and the number of dropouts due to adverse events using risk ratios (RR). We also analysed data at one and up to seven months after treatment.

When ranges of data were presented, we calculated the standard deviations (SD) as advised and checked by the statistician from the Cochrane Musculoskeletal Group (CMSG) according to the Cochrane guidelines. With studies using more than one control arm we combined them as per the Cochrane guidelines (Higgins 2011).

\section{Dealing with missing data}

We contacted the authors of the included/excluded articles to obtain further information. We received responses to queries from the authors of Assefi 2005 (via the last author of the article); Deluze 1992; Harris 2005; Harris 2008; Harris 2009; Itoh 2010; Martin 2006; Sprott 1998; Targino 2008.

\section{Assessment of reporting biases and small sample biases}

For studies published after 1 July 2005, we screened the Clinical Trial Register via the International Clinical Trials Registry Platform of the World Health Organization (http://www.who.int/ ictrp/en/) and compared the outcome measures described in the registry with those reported in the publications to assess whether selective reporting of outcomes was present (outcome reporting bias).

As planned, we compared the fixed-effect estimate against the random-effects model to assess the possible presence of small sample bias in the published literature given that the random-effects estimate of the intervention is more beneficial than the fixed-effect estimate in the presence of small sample bias (Higgins 2011). We found no difference between the two analyses in any outcome measures except for pain under the comparison of acupuncture versus sham acupuncture. The result of the random-effects model was more conservative than the fixed-effect model. Thus, we reported only the results from random-effects model.

If there were sufficient studies ( $>10$ studies with the same outcome), we planned to assess for publication bias using a funnel plot (Sutton 2000). This was not conducted due to an insufficient number of trials. 


\section{Data synthesis}

As recommended by the Cochrane Musculoskeletal Group editor, we used the random-effects model as the default for data synthesis.

\section{Subgroup analyses and assessment of heterogeneity}

When there were sufficient appropriate data, we planned subgroup analyses to assess the effect of different types of acupuncture: 1) manual acupuncture versus electro-acupuncture; 2) shallow needling versus deep needling; 3) different forms of sham/ placebo acupuncture.

We used the $\mathrm{I}^{2}$ statistic to describe the percentage variability of effect estimates that were due to heterogeneity. If there was substantial statistical heterogeneity ( $\mathrm{I}^{2}$ value of $50 \%$ or more) (Higgins 2011), we examined the characteristics of individual studies to determine possible causes.

\section{Sensitivity analyses}

We also planned to conduct sensitivity analyses to examine whether aspects of methodological quality influence the effect size. For example, did inadequate or unclear concealment of allocation or failure to blind outcome assessors change the overall effect estimate of our meta-analysis for pain?

\section{'Summary of findings' tables}

We presented the main outcomes (pain, physical function, global well-being (rated by participants), sleep, fatigue, stiffness and adverse events (Arnold 2011)) in 'Summary of findings' tables. The tables include an overall grading of the evidence using the GRADE approach of high, moderate, low and very low quality:

- High quality: further research is very unlikely to change our confidence in the estimate of effect.

- Moderate quality: further research is likely to have an important impact on our confidence in the estimate of effect and may change the estimate.

- Low quality: further research is very likely to have an important impact on our confidence in the estimate of effect and is likely to change the estimate.

- Very low quality: we are very uncertain about the estimate.

The 'Summary of findings' tables also contain the available data on the main outcomes as the calculations for statistically significant outcomes, and the number needed to treat (NNT) as recommended by The Cochrane Collaboration (Higgins 2011).

For continuous outcomes, we calculated the NNT using the Wells calculator software, available from the Cochrane Musculoskeletal Group editorial office (www.cochranemsk.org), which requires a minimal clinically important difference for input into the calculator. For pain we used a 1.5 -point difference out of a 0 to 10 scale or 15 out of 0 to 100 scale as a minimal clinically important change. For global well-being, we used 14 out of 100 as a minimal clinically important change as recommended by Bennett 2009 for dealing with FIQ data. For sleep, fatigue, stiffness (Martin 2006) and physical function (Harris 2005), we used 13 out of 100 or 1.3 out of 10 as a minimal clinically important change (Bennett 2009). We calculated absolute change (benefit) from the mean difference or standard mean difference and expressed this as a per cent and in the original units, and calculated relative difference in the change from baseline as the absolute benefit divided by the baseline mean of the control group.

\section{RES U L T S}

\section{Description of studies}

Detailed data are summarised in the tables 'Characteristics of included studies' and 'Characteristics of excluded studies'.

\section{Total studies located for this review}

We conducted our initial search in 2008, updated it in May 2010 and then updated it again in January 2012. The search period ranged from the inception of the databases to the end of December 2011. The study selection process is illustrated in Figure 1. The search resulted in 439 studies from the English databases and 63 from Chinese databases. After removing duplicates and irrelevant papers, we identified 49 acupuncture trials for fibromyalgia, including 17 papers in English, 24 in Chinese, four in German, two in Italian, one in Spanish and one in Swedish.

\section{Studies excluded from the review}

We excluded 40 studies for the following reasons:

- Twelve reported number of responders only, without providing any data on the main outcome measures (Guan 2005; Guo 2003; Guo 2005a; Li 2005a; Li 2005; Wang 2002; Wang 2004; Wei 2006; Wu 2003; Yao 2006; Zhang 2001; Zhou 2003).

- Six were conference reports with no useable data (Feldman 2001; Guevara 2007; Harris 2007a; Harris 2007b; Sprott 1995 (translated by the German Cochrane Centre and the author asked us to use his 1998 version); Uhlemann 2001).

- Three had an invalid control (Jiang 2010; Li 2006; Li 2010).

- Three were case series (Chen 2009; Dai 2009; Sun 2008).

- Three had an extra therapy that was not used in the other arm of the trial (Cao 2003; Gong 2010; Gou 2010).

- Four were not randomised; one Spanish (Collazo Chao 2010); one Swedish (Sandberg 1999); two Italian studies stated randomisation in the English abstracts, however the Italian Cochrane Centre, who translated the papers, advised that they were case series (Cassisi 1994; Cassisi 1995). 
- Two did not meet the ACR criteria for fibromyalgia:

(Lautenschlager 1989; Lui 2002).

- Two had insufficient data (Sprott 1998 (could not obtain or confirm the data from the author); Targino 2002).

- Two did not report any of our main outcome measures ( $\mathrm{Li}$ 2008; Sprott 2000).

- One measured blood flow in the muscles upon needling, but did not assess the clinical outcomes (Sandberg 2004).

- One was a secondary analysis of an included trial, the Harris 2005 study (Harris 2006).

- One examined brain images using position emission tomography of participants prior to the acupuncture treatment (Harris 2007).

\section{Studies included in the review}

Nine RCTs and one quasi-RCT were included. Five studies were conducted in the United States of America (Assefi $2005 \mathrm{n}=96$; Harris $2005 \mathrm{n}=56$; Harris $2008 \mathrm{n}=10$; Harris $2009 \mathrm{n}=20$; Martin $2006 \mathrm{n}$ = 49), one in Switzerland (Deluze $1992 \mathrm{n}=55$ ), one in Brazil (Targino $2008 \mathrm{n}=58$ ), one in Japan (Itoh $2010 \mathrm{n}=$ 13) and one quasi-RCT in China (Guo $2005 \mathrm{n}=38$ ). All studies were published in English except for one published in Chinese.

\section{Participants}

In total 395 participants were involved. The authors of the selected papers explained their inclusion and exclusion criteria well except for Guo 2005, which only reported inclusion without exclusion criteria. All studies used acupuncture-naive participants except for Targino 2008, while Guo 2005 did not report this. Targino 2008 admitted participants into their study if they had not received acupuncture in the last 12 months. All studies reported using ACR fibromyalgia criteria for the selection of participants. However, confirmation of the diagnosis before commencement of the studies was reported in only four studies (Assefi 2005; Itoh 2010; Martin 2006; Targino 2008). Assefi 2005 used a researcher trained in tender point examination, Itoh 2010 obtained participants direct from fibromyalgia specialists at hospitals, Martin 2006 used a rheumatologist and Targino 2008 used a physician. The other five studies did not report whether or not they performed a confirmation of diagnosis (Deluze 1992; Guo 2005; Harris 2005; Harris 2008; Harris 2009).

\section{Sample size}

All included studies clearly explained their sample size calculation except for Guo 2005, Harris 2008, Harris 2009 and Itoh 2010. The sample size ranged from four participants to 36 per arm.

\section{Main outcomes}

Main outcome measurement tools varied. Five studies (Assefi 2005; Deluze 1992; Guo 2005; Itoh 2010; Targino 2008) used a VAS for measuring pain. Other measurement tools for pain included Regional Pain Score (Deluze 1992), Numeric Rating Scale (Harris 2005), Multidisciplinary Pain Inventory (Martin 2006) and Short Form of the McGill Pain Questionnaire (Harris 2008; Harris 2009). Three studies measured quality of life (SF-36). Assefi 2005 used SF-36 including the Physical and Mental component, Harris 2005 the SF-36 Physical component, and Targino 2008 reported all eight domains of SF-36. Two studies measured function using the FIQ (Itoh 2010; Martin 2006), which is labelled as global well-being in the current review. Five studies did not include a follow-up phase after the end of the treatment (Deluze 1992; Harris 2005; Harris 2008; Harris 2009; Itoh 2010). The remaining four had follow-ups at different time points with Assefi 2005 at the 3rd and 6th months after the end of the treatment; Guo 2005 at the 6th month; Martin 2006 at the 1st and 7th months; and Targino 2008 at the 3rd, 6th, 12th and 24th months.

\section{Withdrawal/drop-outs}

All studies reported withdrawal, drop-outs or both except for Guo 2005, however the reported data indicated there were no dropouts. The most common reason for withdrawal or drop-out was time constraint, followed by worsening of fibromyalgia symptoms and scheduling conflicts (e.g. appointments). The serious events for discontinuing participation were: one experienced heart attack from the acupuncture group (Assefi 2005); three hospitalisations with one from the acupuncture group and two from the control group; one ankle oedema from the acupuncture group (Deluze 1992), which was the only case that authors reported to be directly related to the acupuncture treatment (ankle oedema). The heart attack and the hospitalisation cases were not explained and connections with interventions were not established. The low dropout rate may suggest the treatments were well tolerated by the participants.

\section{Assessment of the quality of the acupuncture treatments}

\section{STRICTA}

Reporting of the acupuncture treatments was generally adequate with the exception of Guo 2005 and Itoh 2010, being the poorest. Upon our request, some authors provided missing details via emails, however based on the published papers as they were, it would have been impossible to reproduce any of the studies accurately (Appendix 10). 


\section{Adequacy of acupuncture treatment protocol}

Reporting of the rationale of the acupuncture treatment was insufficient, making it difficult for us to give a rating. Considering all 11 criteria, we rated the studies as 'medium' for all studies except for Guo 2005 and Itoh 2010, which we noted as 'insufficient data'. Six studies (Deluze 1992; Guo 2005; Harris 2005; Harris 2009; Itoh 2010; Martin 2006) actually stated the acupuncture style, while the rest did not report the style. Based on how they reported the acupuncture treatment, we inferred that it was 'formula acupuncture' (symptom alleviation), using a set of fixed acupuncture points.

None of the studies stated whether they had made a diagnosis according to Chinese medicine, except for Deluze 1992, but there was insufficient information to confirm this. Deluze 1992 stated that they individualised treatment and provided references to support their decision. However, the authors failed to report their Chinese medicine diagnosis.

The justification of acupuncture point selection deserved some attention. Only one study provided journal references for their decision on point selection and choice of electro-acupuncture (Deluze 1992). This study reported, however, only two mandatory acupuncture points without detailing the other eight optional points. Assefi 2005 commented that no gold standard existed for acupuncture point selection in the treatment of fibromyalgia, with the authors consulting three experienced acupuncturists in treating fibromyalgia for their point selection. Guo 2005 did not state what acupuncture points were used, instead naming the channels/ meridians they used. They considered 'Back Shu' points along the Bladder meridian important to strengthen Liver, Spleen and Kidney when treating $B i$-Syndrome, a Chinese medicine term for a series of rheumatic conditions, including fibromyalgia. Harris 2005 chose acupuncture points based on their "ability to relieve fibromyalgia symptoms in CM", however this was referenced to a textbook that does not specify Chinese medicine treatments for fibromyalgia. Harris 2008 and Harris 2009 referenced their 2005 trial for their acupuncture point selection. Itoh 2010 did not explain. Martin 2006 stated they used "strong regulatory points that commonly recur in acupuncture literature", yet provided neither reference nor stated the acupuncture points used on the back (published a small diagram of areas used). They also stated that their acupuncture point selection might not be optimal as judged by others, but did not provide the reason. Targino 2008 used "classical acupuncture points" and they referenced an acupuncture point location book that does not include fibromyalgia. The most commonly used point in all included studies was $\mathrm{He} G u$ (LI4), followed by Zu San Li (ST36).

With the reporting of unilateral/bilateral needling details, Assefi 2005, Deluze 1992, Guo 2005 and Itoh 2010 did not state which side they inserted the needles on, while Martin 2006, Harris 2005 and Harris 2009 provided diagrams. Harris 2008 based the treatment on their 2005 trial. Except for Deluze 1992, Guo 2005 and Itoh 2010 the number of needles used was clearly stated. Reporting the depth of needle insertion was clear in all studies except for Guo 2005, which we thought was subcutaneous because the needling technique was "point to point threading" along the back meridian/channels. Reporting of elicitation of deqi was clear except for Assefi 2005, Guo 2005 and Itoh 2010; two stated "stimulation" without mentioning deqi (Assefi 2005; Itoh 2010) and the other did not report this (Guo 2005). The description of the type of needle stimulation/manipulation (e.g. lifting/thrusting/even etc.) was clear in only three studies (Harris 2005; Harris 2009; Itoh 2010). With the electro-acupuncture studies (Deluze 1992; Itoh 2010; Martin 2006) both reported Hz but not where the red/black clips went or what type of stimulation setting was used, such as 'continuous'. Needle gauge/length/manufacturer or material varied greatly and were not well reported by some.

Needle retention time ranged from 20 to 30 minutes. Four studies treated the participants for 30 minutes (Assefi 2005; Guo 2005; Harris 2005; Itoh 2010), two for 25 minutes (Harris 2008; Harris 2009) and two for 20 minutes (Martin 2006; Targino 2008). One did not report the needling duration (Deluze 1992). The median duration of acupuncture treatment sessions was four weeks (range 3 to 13). Two had six sessions (Deluze 1992; Martin 2006), with the remainder, nine (Harris 2008; Harris 2009), 10 (Itoh 2010), 18 (Harris 2005), 20 (Targino 2008), 24 (Assefi 2005) and 28 sessions (Guo 2005). Itoh 2010 was a cross-over study and we used data before cross-over for analysis; that is after five sessions of treatment. Frequency of treatments was similar in most studies, with twice weekly being the commonest (Assefi 2005; Deluze 1992; Martin 2006; Targino 2008). Two trials (Harris 2008; Harris 2009) had nine sessions over four weeks, another (Harris 2005) gave 18 sessions over 13 weeks, while one (Itoh 2010) delivered weekly and the remainder (Guo 2005) daily treatment.

\section{Confidence in the treatment delivery}

We rated our confidence that acupuncture treatments were appropriately delivered by skilled practitioners as 'high' for Assefi 2005 and Harris 2005 and 'medium' for Targino 2008. The remaining studies (Deluze 1992; Guo 2005; Harris 2008; Harris 2009; Itoh 2010; Martin 2006) we noted as 'insufficient data'.

\section{Adverse events}

Reporting of adverse events was inconsistent. Only two studies provided details of the number of events (Assefi 2005; Targino 2008). With the remaining studies, three did not report any (Harris 2005; Harris 2008; Harris 2009), which the author confirmed as nil. Two (Deluze 1992; Itoh 2010) cited them as withdrawals, one (Martin 2006) discussed them in the results without labelling them as adverse events and one (Guo 2005) did not report any.

None of the studies reported serious adverse events. The worst events that could directly be attributed to an acupuncture treatment were oedema of the left hand and ankle, despite a lack of 
evidence of a causal relationship (Targino 2008). Two cases of vasovagal symptoms reported by Martin 2006 were likely due to the posture of the participants. The author did not explain which treatment group the cases were in. In that study, all participants received acupuncture treatment seated without a back support for 20 minutes, which is not a common practice (participants are mostly in a prone or supine position), however it allowed the participants to be blinded. This type of adverse event could be prevented or reduced with correct posture as suggested by the author.

\section{Subgroup analyses}

We undertook subgroup analyses within the comparison of acupuncture versus placebo/sham acupuncture to compare electro- and manual acupuncture for the outcome of pain, fatigue, sleep and global well-being (rated by participants) as data were available for those outcome measures. We also undertook a subgroup analyses of studies using sham acupuncture without breaking the skin (Assefi 2005; Harris 2008; Harris 2009; Martin 2006) versus studies using breaking-skin sham interventions (Assefi 2005; Deluze 1992; Harris 2005). One arm of the control (simulating) in Assefi 2005 and the sham controls in Harris 2008 and Harris 2009 used a tooth pick in an acupuncture guide tube, which has been shown in other studies of back pain to be indistinguishable (Sherman 2002). Martin 2006 indented the skin with a blunt probe and placed over the area a small circular plaster rigged with an acupuncture needle that stuck out; they stated that preliminary trials showed volunteers could not tell the difference.

\section{Publication bias}

We did not perform the planned publication bias funnel plot analysis due to an insufficient number of selected studies (Sutton 2000).

\section{Sensitivity analyses}

We could not conduct planned sensitivity analyses due to a lack of trials with and without adequate concealment of allocation; or with and without blinded outcome assessor under one comparison. For instance, under the comparison of real and sham acupuncture, all six studies were at low risk of selection bias with adequate concealment of treatment allocation, using no treatment as the control, and at low risk of detection bias with outcome assessors being blinded (Assefi 2005; Deluze 1992; Harris 2005; Harris 2008; Harris 2009; Itoh 2010; Martin 2006). Two studies were at higher risk of selection bias with unclear or inadequate allocation concealment and had a high risk of detection bias with inadequate or unclear blinding of outcome assessor (Guo 2005; Targino 2008). They were, however, under different comparison categories and contained only one study in each. Itoh 2010 had a moderate risk of bias as a non-acupuncture treatment control was used.

\section{Risk of bias in included studies}

All studies were described as RCTs. Adequate sequence generation and allocation concealment were well described and adequate in all included studies except for Guo 2005, which used order of admission for randomisation (quasi-randomisation) and Martin 2006, which did not say how the sequence was generated. All studies used acupuncture-naive participants except for Guo 2005 (who did not report this) and Targino 2008 (patients had not had acupuncture in the last 12 months). Four studies tested for assessment of masking/blinding (Assefi 2005; Harris 2005; Harris 2009; Martin 2006) and found no difference between groups. Five studies blindfolded their participants (Assefi 2005; Harris 2005; Harris 2008; Harris 2009), while Martin 2006 blocked the vision of the participants. All used blinded assessors except for Guo 2005, which did not report this. All studies showed no missing data except for Deluze 1992 and Itoh 2010, which did not include participants who dropped out from the study in their data analysis, while Guo 2005 did not report this specifically. All reported numbers lost to follow-up except for Guo 2005, which did not report this but no participant was missing from the reported data. In terms of selective reporting, only Assefi 2005, Harris 2005 and Targino 2008 were registered with the International Clinical Trials Registry Platform of the World Health Organization and we found that the outcome measures reported were same as those included in the published protocols. Among the remainder, one did not know about it (Martin 2006), one did not need to report it as it was before the establishment of the Registry (Deluze 1992), whereas the others did not list this (Guo 2005; Harris 2008; Harris 2009; Itoh 2010) (Figure 2; Figure 3). 
Figure 2. Methodological quality summary: review authors' judgements about each methodological quality item for each included study.

\begin{tabular}{|c|c|c|c|c|c|c|}
\hline & 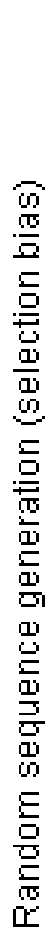 & 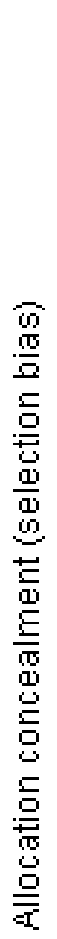 & 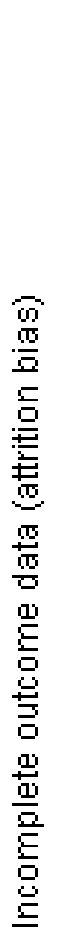 & 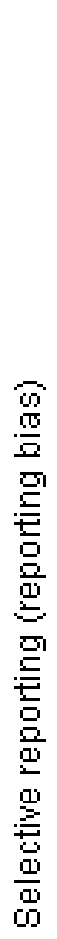 & 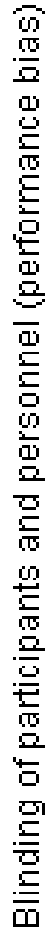 & 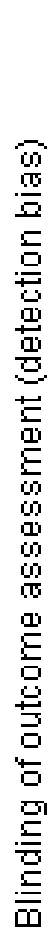 \\
\hline Assefi 2005 & & & & & & \\
\hline Deluze 1992 & & & & & & \\
\hline Guo 2005 & & & & $?$ & $?$ & $?$ \\
\hline Harris 2005 & & & & & & \\
\hline Harris 2008 & & & & $?$ & & \\
\hline Harris 2009 & & & & $?$ & & \\
\hline Itoh 2010 & & & & $?$ & & \\
\hline Martin 2006 & $?$ & & & $?$ & & \\
\hline Targino 2008 & & & & & & \\
\hline
\end{tabular}


Figure 3. Methodological quality graph: review authors' judgements about each methodological quality item presented as percentages across all included studies.

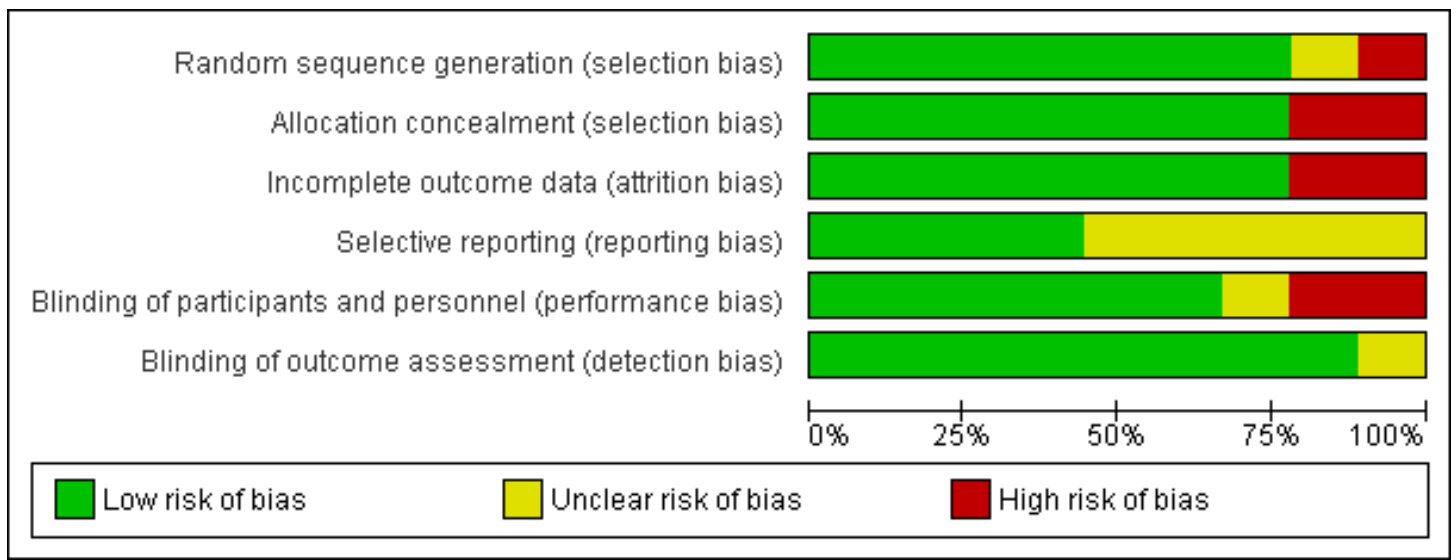

\section{Effects of interventions}

See: Summary of findings for the main comparison Acupuncture versus non-acupuncture for treating fibromyalgia; Summary of findings 2 Acupuncture versus placebo or sham acupuncture for treating fibromyalgia; Summary of findings 3 Acupuncture versus medication for treating fibromyalgia; Summary of findings 4 Acupuncture as an adjunct therapy for treating fibromyalgia; Summary of findings 5 Deep invasive acupuncture stimulation versus non-stimulated acupuncture for treating fibromyalgia

\section{I) Real acupuncture versus non-acupuncture treatment}

One study in this category (Itoh 2010) included 13 participants and compared electro-acupuncture plus trigger point acupuncture with no acupuncture treatment. This was a cross-over study, and we used data before cross-over for analysis, that is after five sessions of treatment at the end of week five of a 10 -week treatment programme.

\section{Main outcome measure I: Pain}

Pain severity was measured using a VAS $(100 \mathrm{~mm})$. It showed a statistically significant reduction in pain for those treated with real acupuncture compared with no acupuncture at the end of treatment (mean difference (MD) -22.40 points on a 100-point scale; $95 \%$ confidence interval $(\mathrm{CI})-40.98$ to $-3.82, \mathrm{P}=0.02)$, favouring acupuncture (Analysis 1.1).

\section{Main outcome measure 2: Global well-being; rated by participant}

Global well-being was measured using the Fibromyalgia Impact Questionnaire (FIQ) 100-point scale. It showed a statistically significant group difference at the end of treatment (MD -15.40 points on a 100 -point scale; $95 \% \mathrm{CI}-25.62$ to $-5.18, \mathrm{P}=0.003)$, favouring acupuncture (Analysis 1.2).

\section{Main outcome measure 3: Sleep}

Sleep was measured using the subset 'rested' on the FIQ. It showed no statistically significant group difference at the end of treatment (MD - 0.40 points on a 10 -point scale; $95 \% \mathrm{CI}-1.01$ to 0.21 , P $=0.20$ ) (Analysis 1.3).

\section{Main outcome measure 4: Fatigue}

Fatigue was measured using the subset 'fatigue' on the FIQ. It showed a statistically significant group difference at the end of treatment (MD -1.10 points on a 10-point scale; $95 \% \mathrm{CI}-1.98$ to $-0.22, \mathrm{P}=0.01$ ), favouring acupuncture (Analysis 1.4).

\section{Main outcome measure 5: Stiffness}

Stiffness was measured using the subset 'stiffness' on the FIQ. It showed a statistically significant group difference at the end of treatment (MD - 0.90 points on a 10-point scale; $95 \% \mathrm{CI}-1.66$ to $-0.14, \mathrm{P}=0.02$ ), favouring acupuncture (Analysis 1.5). 


\section{Main outcome measure 6: Adverse events}

No adverse events were reported, only withdrawals and drop-outs. One patient from the acupuncture group and two from the control group left the study as their condition was not improving.

\section{Minor outcome measure I: Mental well-being}

Mental well-being was measured using the subset 'depression' on the FIQ. It showed no statistically significant group difference at the end of treatment (MD - 0.50 points on a 10-point scale; $95 \%$ CI -1.10 to $0.10, \mathrm{P}=0.10$ ) (Analysis 1.7 ).

\section{Long-term effect of acupuncture}

There was no follow-up and long-term effect was not measured.

\section{2) Real acupuncture versus placebo or sham acupuncture}

\section{Main outcome measure I: Pain up to one month after treatment}

Six studies totaling 286 participants were in this category (Assefi 2005; Deluze 1992; Harris 2005; Harris 2008; Harris 2009; Martin 2006). Measurement tools used included VAS (0 to $10 \mathrm{~cm}$ and 0 to $100 \mathrm{~mm}$ ), numerical pain rating scale (NRS), Multidimensional Pain Inventory (MPI) and McGill Pain Questionnaire (SF-MPQ). Pooled analysis of the six studies showed no statistically significant difference between the groups in reducing pain (standardised mean difference (SMD) $-0.14 ; 95 \%$ CI -0.53 to $0.25, \mathrm{P}=0.48$; corresponding to a reduction of 2.8 points on a 100 -point scale) (Analysis 2.1). Moderate heterogeneity was found $\left(\mathrm{I}^{2}=54 \%, \mathrm{P}=0.05\right)$ and is likely due to the two forms of acupuncture, electro- and manual, employed in the different studies.

Pain subgroup analysis (electro- versus manual acupuncture) Pooled subgroup analysis of two electro-acupuncture studies, including 104 participants (Deluze 1992; Martin 2006), indicated that real electro-acupuncture was statistically significantly better than sham electro-acupuncture in reducing pain (SMD -0.63; $95 \% \mathrm{CI}-1.02$ to $-0.23, \mathrm{P}=0.002$, about 13 points on a 100 point scale) (Analysis 2.1) up to one month after treatment, with low heterogeneity $\left(\mathrm{I}^{2}=0 \%, \mathrm{P}=0.72\right)$. Subgroup analysis of four manual acupuncture studies, including 182 participants (Assefi 2005; Harris 2005; Harris 2008; Harris 2009) showed no group difference between real and sham manual acupuncture in reducing pain (SMD 0.14; 95\% CI -0.17 to $0.45, \mathrm{P}=0.37,2.8$ points on a 100-point scale) (Analysis 2.1$)$, with no heterogeneity $\left(\mathrm{I}^{2}=\right.$ $0 \%, \mathrm{P}=0.57)$. There was a statistically significant subgroup difference between electro- and manual acupuncture $\left(\mathrm{Chi}^{2}=8.94\right.$, $\mathrm{P}=0.003)$.
Main outcome measure 2: Physical function (SF-36) up to one month after treatment

One study totaling 56 participants was in this category (Harris 2005). Physical function was measured with the SF-36. Analysis of the study indicated that sham manual acupuncture was superior to manual acupuncture in improving SF-36 physical function (MD -5.80 points on a 100 -point scale; $95 \% \mathrm{CI}-10.91$ to $-0.69, \mathrm{P}=$ 0.03 , Analysis 2.4).

\section{Main outcome measure 3: Global well-being: rated by participants up to one month after treatment}

Three studies totaling 200 participants were in this category (Assefi 2005; Deluze 1992; Martin 2006). Measurement tools included VAS and FIQ. Pooled analysis of the three studies showed no statistically significant difference between real and sham acupuncture (SMD 0.29 ; $95 \%$ CI -0.44 to $1.01, \mathrm{P}=0.44,5.8$ points on a 100 point scale) (Analysis 2.5), with high heterogeneity $\left(\mathrm{I}^{2}=81 \%, \mathrm{P}=\right.$ $0.003)$. This is likely due to the two forms of acupuncture, electroand manual, employed in the different studies.

Global well-being subgroup analysis (electro-acupuncture versus manual acupuncture)

Pooled subgroup analysis of two electro-acupuncture studies with 104 participants (Deluze 1992; Martin 2006) indicated that real electro-acupuncture was statistically significantly better than sham electro-acupuncture in reducing global well-being as rated by participants (SMD 0.65; 95\% CI 0.26 to $1.05, \mathrm{P}=0.001$, about 11 points on a 100-point scale) (Analysis 2.5), up to one month after treatment, with low heterogeneity $\left(\mathrm{I}^{2}=0 \%, \mathrm{P}=0.99\right)$. Subgroup analysis of one manual acupuncture study of 96 participants (Assefi 2005) showed no difference between real and sham manual acupuncture (SMD -0.40; 95\% CI -0.86 to $0.06, \mathrm{P}=0.09$, about eight points worse on a 100-point scale) (Analysis 2.5). Subgroup comparison indicated that electro-acupuncture was statistically significantly better than manual acupuncture in improving global well-being as rated by participants up to one month after treatment $\left(\mathrm{Chi}^{2}=11.49, \mathrm{P}=0.0007\right)$.

\section{Main outcome measure 4: Sleep up to one month after treatment}

Three studies totaling 200 participants were in this category (Assefi 2005; Deluze 1992; Martin 2006). Sleep quality was measured with a VAS sleep scale and the subset 'rested' on the FIQ. Pooled analysis showed no statistically significant difference with real acupuncture when compared with sham interventions (SMD 0.16 ; $95 \% \mathrm{CI}-0.29$ to $0.61, \mathrm{P}=0.49$, about 3.2 points on a 100 -point scale) (Analysis 2.7), with moderate heterogeneity ( $\mathrm{I}^{2}$ $=56 \%, \mathrm{P}=0.10)$. 
Sleep subgroup analysis (electro-acupuncture versus manual acupuncture)

Pooled subgroup analysis of two electro-acupuncture studies with 104 participants (Deluze 1992; Martin 2006) indicated that real electro-acupuncture was statistically significantly better than sham electro-acupuncture in improving sleep quality (SMD 0.40; 95\% CI 0.01 to $0.79, \mathrm{P}=0.05$, about eight points on a 100 -point scale) (Analysis 2.7) up to one month after treatment, with low heterogeneity $\left(\mathrm{I}^{2}=0 \%, \mathrm{P}=0.74\right)$. Subgroup analysis of one manual acupuncture study with 96 participants (Assefi 2005) showed no difference between real and sham manual acupuncture in improving sleep (SMD -0.25; 95\% CI -0.71 to $0.21, \mathrm{P}=0.29$, five points worse on a 100-point scale) (Analysis 2.7). Subgroup comparison indicated that electro-acupuncture was statistically significantly better than manual acupuncture in improving sleep up to one month after treatment $\left(\mathrm{Chi}^{2}=4.44, \mathrm{P}=0.04\right)$.

\section{Main outcome measure 5: Fatigue up to one month after treatment}

Three studies totaling 201 participants were in this category (Assefi 2005; Harris 2005; Martin 2006). Fatigue was measured with a VAS, the Multidimensional Fatigue Inventory (MFI) and the subset 'fatigue' on the FIQ. Pooled analysis showed no statistically significant difference between real and sham acupuncture in reducing fatigue (SMD $-0.10 ; 95 \% \mathrm{CI}-0.81$ to $0.61, \mathrm{P}=0.78$, about 1.7 points on a 100-point scale, Analysis 2.9), with high heterogeneity $\left(\mathrm{I}^{2}=82 \%, \mathrm{P}=0.004\right)$. This is likely due to the two forms of acupuncture, electro- and manual, being employed in the different studies.

Fatigue subgroup analysis (electro-acupuncture versus manual acupuncture)

Subgroup analysis of one electro-acupuncture study (Martin 2006) of 49 participants indicated that real electro-acupuncture was statistically significantly better than sham electro-acupuncture in reducing fatigue (SMD -0.85; 95\% CI -1.44 to $-0.27, \mathrm{P}=0.004$, about 15.3 points on a 100 -point scale) (Analysis 2.9) up to one month after treatment. Pooled subgroup analysis of two manual acupuncture studies (Assefi 2005; Harris 2005) with 152 participants showed no group difference between real and sham manual acupuncture in reducing fatigue (SMD 0.26; 95\% CI -0.08 to $0.61, \mathrm{P}=0.13$, about 4.3 points worse on a 100 -point scale) (Analysis 2.9), with low heterogeneity $\left(\mathrm{I}^{2}=0 \%, \mathrm{P}=0.39\right)$. Subgroup comparison indicated that electro-acupuncture was statistically significantly better than manual acupuncture in improving fatigue up to one month after treatment $\left(\mathrm{Chi}^{2}=10.31, \mathrm{P}=0.001\right)$.

\section{Main outcome measure 6: Stiffness up to one month after treatment}

Two studies totaling 104 participants using electro-acupuncture treatment (Deluze 1992; Martin 2006) were in this category. Stiffness was measured as minutes (Deluze 1992) and the subset 'stiffness' on the FIQ (Martin 2006). Pooled analysis showed that real electro-acupuncture was statistically significantly better than sham electro-acupuncture in improving stiffness (SMD - 0.45; 95\% CI 0.84 to $-0.06, \mathrm{P}=0.02$, nine points on a 100 -point scale) (Analysis 2.11) up to one month after treatment, with low heterogeneity (I $2=0 \%, P=0.42)$.

\section{Main outcome measure 7: Adverse events}

There were no serious adverse events reported. Minor adverse events were reported in 11 out of 113 participants in the acupuncture groups and 58 out of 156 in the control groups (risk ratio (RR) 0.44 ; $95 \%$ CI 0.12 to $1.63, \mathrm{P}=0.22$, Analysis 2.13 ), with moderate heterogeneity $\left(\mathrm{I}^{2}=67 \%, \mathrm{P}=0.05\right)$. This could be due to the three sham groups in one study (Assefi 2005) reporting over $60 \%$ adverse events; this was much higher than the other studies, which were typically under $30 \%$.

\section{Minor outcome I: Mental well-being up to one month after treatment}

One study totaling 49 participants using electro-acupuncture was in this category (Martin 2006). Mental well-being was measured with the subset 'depression' of the FIQ. Analysis showed a statistically significantly better result with real electro-acupuncture when compared to sham electro-acupuncture in improving mental wellbeing (MD -1.70 points on a 10-point scale; $95 \%$ CI -3.13 to $0.27, \mathrm{P}=0.02$ ) (Analysis 2.14), up to one month after treatment.

\section{Minor outcome measure 2: Analgesic use (number of tablets) up to one month after treatment}

One study with 55 participants using electro-acupuncture treatment (Deluze 1992) measured analgesic use by the number of tablets per week. There was no difference between real and sham electro-acupuncture (MD -3.20 tablets less per week; 95\% CI 10.20 to $3.80, \mathrm{P}=0.37$ ) (Analysis 2.16 ).

\section{Minor outcome measure 3: Analgesic use (number of participants) up to one month after treatment}

One study with 80 participants using manual acupuncture treatment (Assefi 2005) measured analgesic use by number of participants taking analgesics. There was no difference between real and sham manual acupuncture (RR 0.94; 95\% CI 0.66 to $1.32, \mathrm{P}=$ 0.71) (Analysis 2.17). 
Minor outcome 4: Tenderness up to one month after treatment

One study with 55 participants using electro-acupuncture treatment (Deluze 1992) measured pressure pain threshold $\left(\mathrm{kg} / \mathrm{cm}^{2}\right)$. Electro-acupuncture was better than sham electro-acupuncture in enhancing pain thresholds (MD $0.80 \mathrm{~kg} / \mathrm{cm}^{2}$ higher; $95 \% \mathrm{CI}$ 0.02 to $1.58, \mathrm{P}=0.04$ ) (Analysis 2.18 ), up to one month after treatment.

Minor outcome measure 5: Overall well-being: rated by care giver

One study with 55 participants using electro-acupuncture treatment (Deluze 1992) reported overall well-being rated by care givers. Analysis showed a statistically significantly better result with real electro-acupuncture when compared with sham electroacupuncture (MD 2.00 points on a 10-point scale; $95 \%$ CI 0.81 to $3.19, \mathrm{P}=0.001$ ) (Analysis 2.19 ), up to one month after treatment.

\section{Long-term effects of acupuncture}

Two studies (Assefi 2005; Martin 2006) measured long-term effects of acupuncture for up to seven months after the end of the treatment. There was no difference between real and sham acupuncture on any outcome measures, including pain (Analysis 2.2), global well-being (Analysis 2.6), sleep (Analysis 2.8), fatigue (Analysis 2.10), stiffness (Analysis 2.12) and mental well-being (Analysis 2.15). Subgroup comparison indicated that electroacupuncture was not statistically significantly better than manual acupuncture at improving any of the outcomes at seven months after treatment.

\section{Sham non-invasive (not breaking skin) acupuncture versus sham invasive (breaking skin) acupuncture}

Four studies (Assefi 2005; Harris 2008; Harris 2009; Martin 2006) with 116 participants using non-invasive sham acupuncture were compared with three studies (Assefi 2005; Harris 2005; Deluze 1992) with 170 participants using invasive sham interventions. There was no statistically significant difference between the two subgroups on pain rating $\left(\mathrm{Chi}^{2}=0.40, \mathrm{P}=0.53\right.$, Analysis 2.3).

\section{3) Real acupuncture versus standard or usual care (medication)}

One study in this category (Guo 2005) included 38 participants and compared manual acupuncture with Western medicine (amitriptyline).
Main outcome measure I: Pain at up to one month after treatment

Pain severity was measured using a VAS. It showed a statistically significant group difference favouring acupuncture (MD 17.30 points on a 100 -point scale; $95 \%$ CI -24.13 to $-10.47, \mathrm{P}<$ 0.00001) (Analysis 3.1).

\section{Main outcome measure 2: Adverse events}

No adverse events were reported, however all participants were included in the final analyses. No withdrawals or drop-outs were reported either.

Minor outcome measure I: Number of tender points at up to one month after treatment

A statistically significant group difference was shown for number of tender points, favouring acupuncture (MD -4.00 number of tender points; 95\% CI -6.73 to $-1.27, \mathrm{P}=0.004$ ) (Analysis 3.3).

\section{Long-term effect of acupuncture at the sixth month after treatment}

The authors stated there was follow-up at six months but no data were provided.

The poor reporting of the trial raises questions about its quality. For example, the authors claimed 'cure' of 12 fibromyalgia participants, with nine in the acupuncture group and three in the control, without a definition of what 'cure' was, except for saying "signs and symptoms free with no tender point" without further explanation as to which time point these were measured at.

\section{4) Real acupuncture as an adjunct therapy}

One study in this category (Targino 2008) with 58 participants compared manual acupuncture plus standard therapy, which included tricyclic antidepressants and exercise, with standard therapy alone.

\section{Main outcome measure I: Pain at up to one month after} treatment

Pain severity was measured using a VAS. It showed a statistically significant group difference favouring acupuncture (MD -3.00 points on a 10 -point scale; $95 \% \mathrm{CI}-3.90$ to $-2.10, \mathrm{P}<0.00001$ ) (Analysis 4.1).

\section{Main outcome measure 2: Adverse events}

There were no serious adverse events reported. No group difference in the number of minor adverse events was found (RR 3.57; 95\% CI 0.18 to $71.21, \mathrm{P}=0.40$ ) (Analysis 4.3). 
Minor outcome measure I: Tenderness - number of tender points below $\mathrm{kg} / \mathrm{cm}^{2}$ up to one month after treatment

Tenderness was measured the number of tender points below the threshold. The results showed a statistically significant group difference favouring acupuncture (MD -4.50 number of tender points; 95\% CI -6.20 to $-2.80, \mathrm{P}<0.00001$ ) (Analysis 4.4).

Minor outcome measure 2: Tenderness - mean pressure threshold by pressure algometry at end of treatment

Tenderness was measured with the mean pressure threshold $(\mathrm{kg} /$ $\mathrm{cm}^{2}$ ). The results showed a statistically significant group difference (MD $0.70 \mathrm{~kg} / \mathrm{cm}^{2}$; 95\% CI 0.41 to $0.99, \mathrm{P}<0.00001$ ) (Analysis 4.6), favouring acupuncture.

\section{Long-term effect of acupuncture (follow-up at six months)}

At the six-month follow-up, the acupuncture as an adjunct therapy group continued to be better than the standard therapy alone group for tender points (MD -2.00 number of tender points; $95 \%$ $\mathrm{CI}-3.51$ to $-0.49, \mathrm{P}=0.009$ ) (Analysis 4.5 ) and mean pressure pain threshold (MD $0.60 \mathrm{~kg} / \mathrm{cm}^{2} ; 95 \%$ CI 0.26 to $0.94, \mathrm{P}=$ 0.0005 ) (Analysis 4.7) but not pain (MD - 0.50 points on a 10 point scale; $95 \% \mathrm{CI}-1.49$ to $0.49, \mathrm{P}=0.37$ ) (Analysis 4.2).

5) A particular style of acupuncture versus another (deep invasive needling with stimulation (deqi) (T/S)

\section{versus deep invasive needling without stimulation} (T/O))

Two different styles of acupuncture, deep needling using manual acupuncture on the point with stimulation to achieve deqi as in traditional Chinese acupuncture (T/S) versus deep needling on the point without stimulation $(\mathrm{T} / \mathrm{O})$, were compared in one study of 41 participants (Harris 2005).

\section{Main outcome measure I: Pain at the end of the treatment}

Pain was measured using the NRS. It showed no statistically significant difference between the two interventions (MD 0.30 on a 10-point scale; $95 \% \mathrm{CI}-18.34$ to $18.94, \mathrm{P}=0.97$ ) (Analysis 5.1).

\section{Main outcome measure 2: Physical function (SF-36) at the} end of the treatment

Physical function was measured using the SF-36 (physical). There was no group difference between the two interventions (MD -5.50 points on a 100 -point scale; $95 \% \mathrm{CI}-11.43$ to $0.43, \mathrm{P}=0.07$ ) (Analysis 5.2).

Main outcome measure 3: Fatigue at the end of the treatment

Fatigue was measured using the MFI. There was no group difference between the two interventions (MD 1.10 points on 20-point scale; 95\% CI -1.41 to 3.61, $\mathrm{P}=0.39$ ) (Analysis 5.3). 


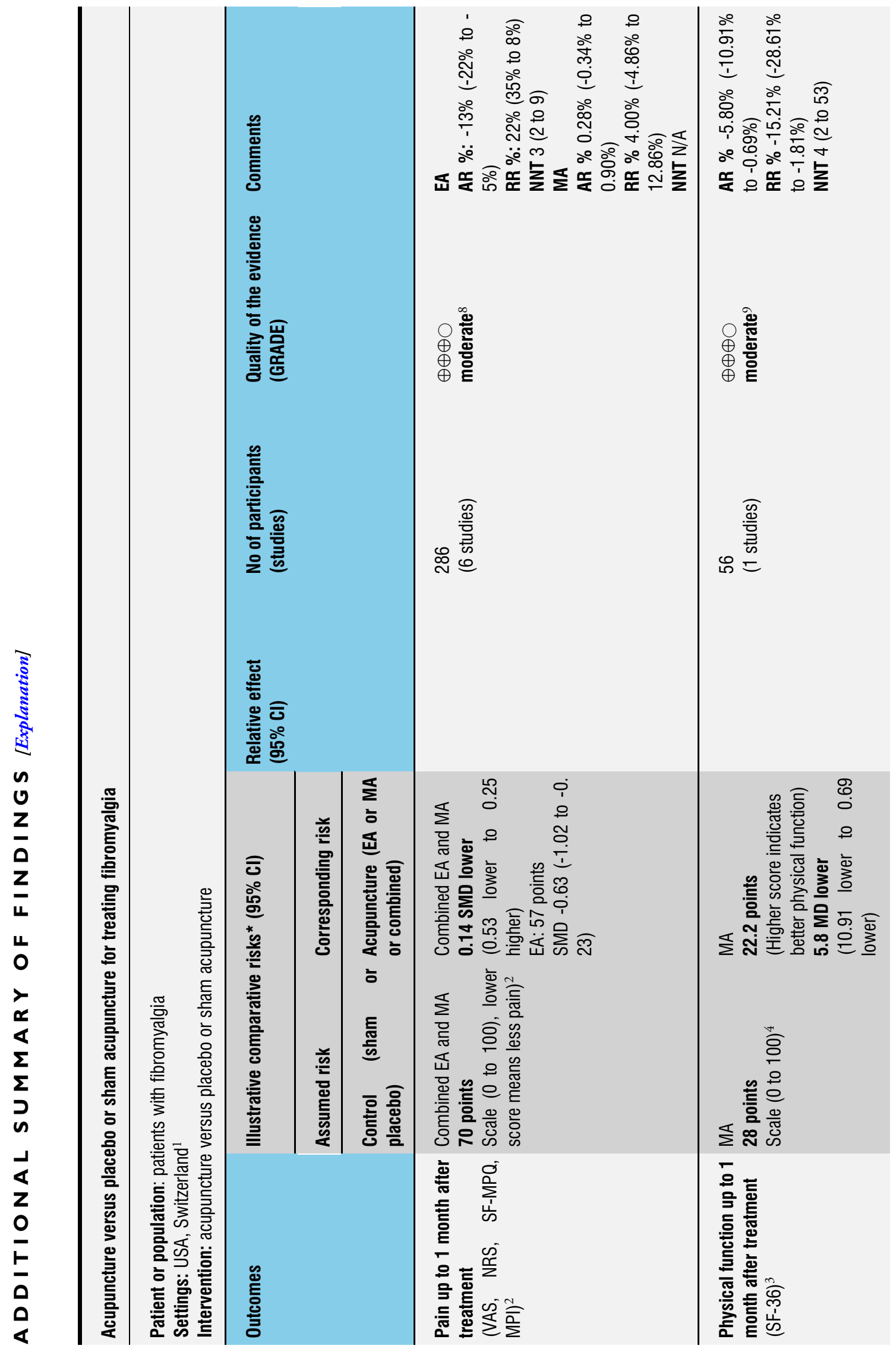

Acupuncture for treating fibromyalgia (Review)

Copyright @ 2013 The Cochrane Collaboration. Published by John Wiley \& Sons, Ltd. 


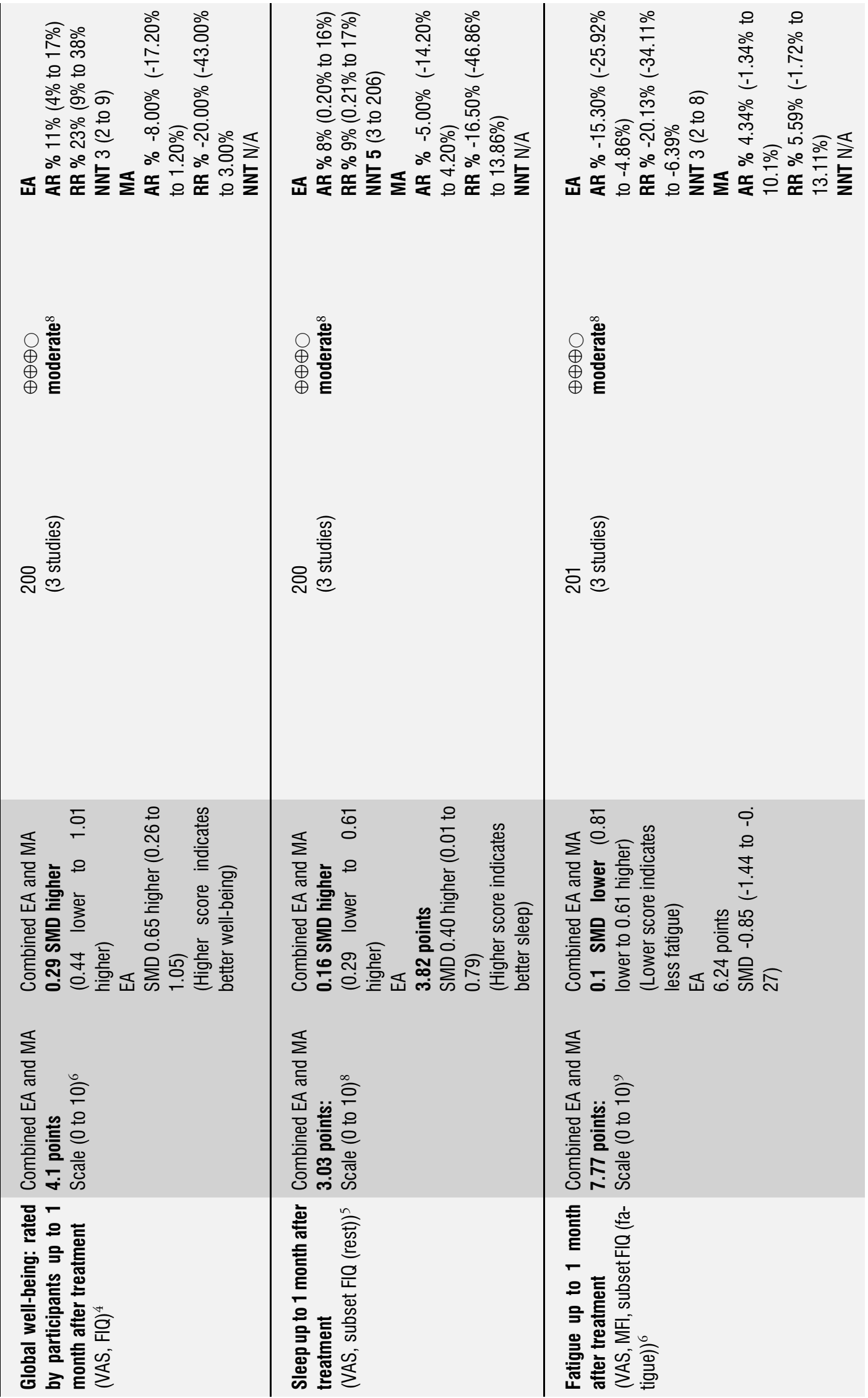

Acupuncture for treating fibromyalgia (Review)

Copyright $₫ 2013$ The Cochrane Collaboration. Published by John Wiley \& Sons, Ltd. 


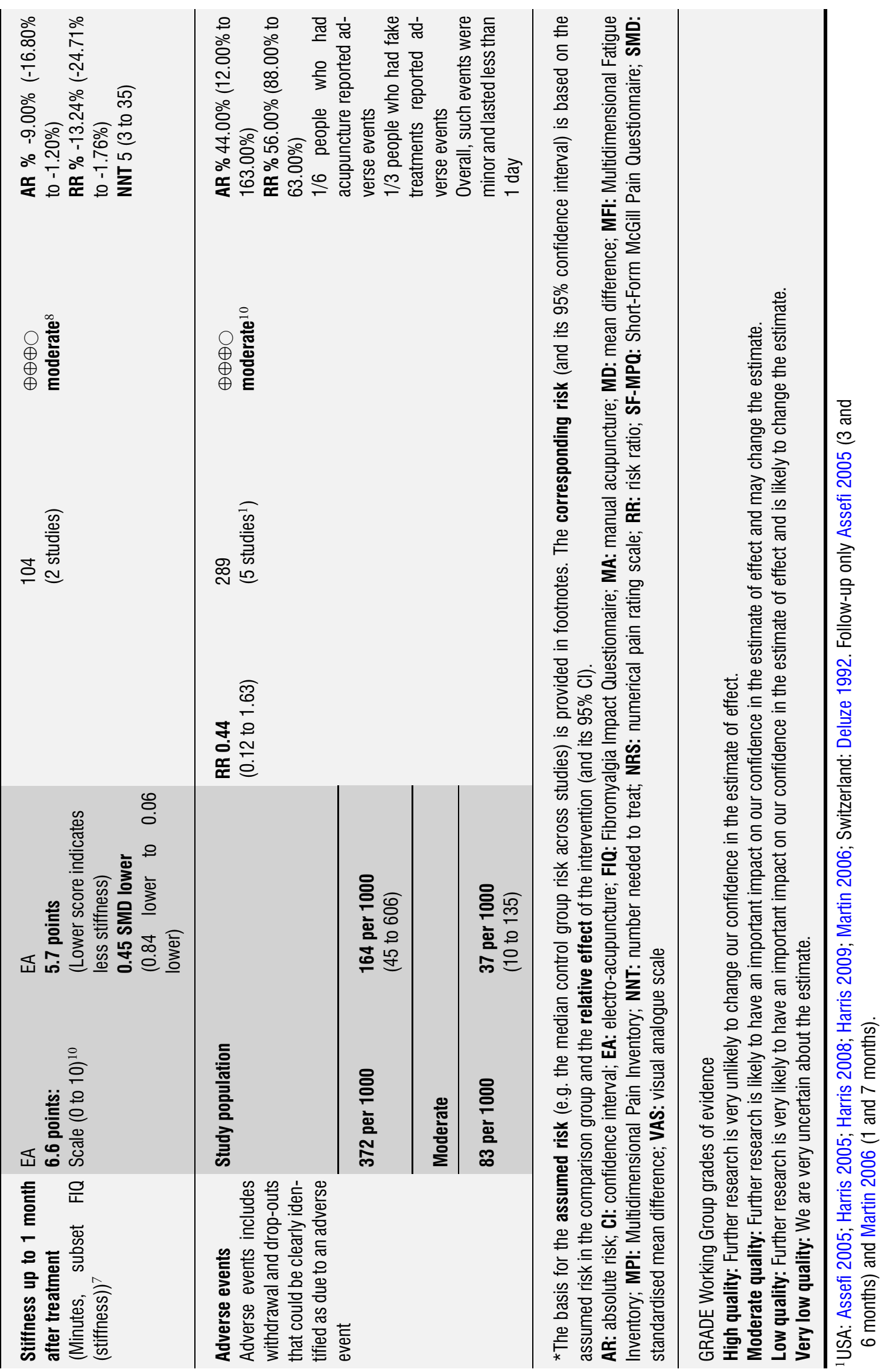

Acupuncture for treating fibromyalgia (Review)

Copyright $\odot 2013$ The Cochrane Collaboration. Published by John Wiley \& Sons, Ltd. 


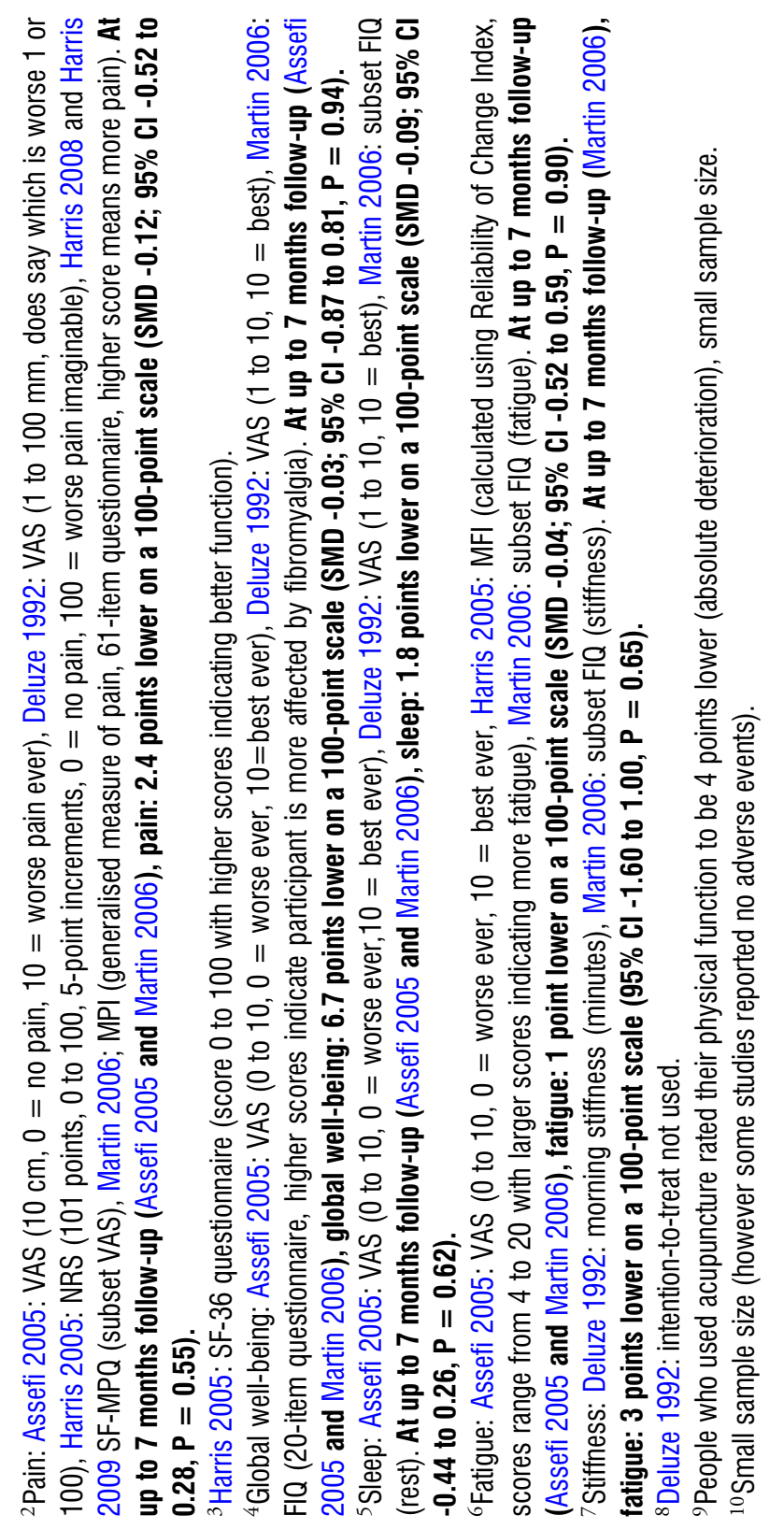

Acupuncture for treating fibromyalgia (Review)

Copyright $\odot 2013$ The Cochrane Collaboration. Published by John Wiley \& Sons, Ltd. 


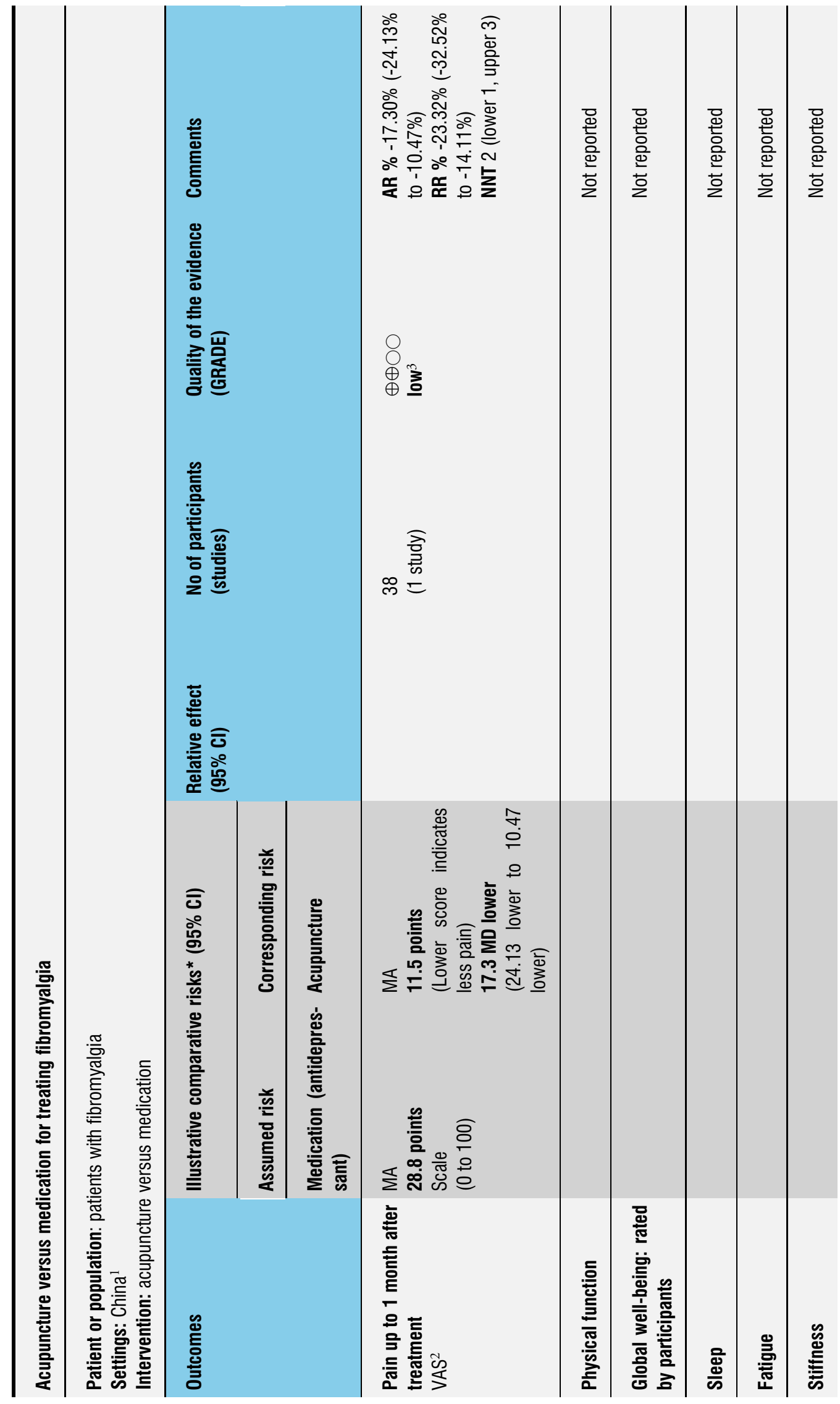




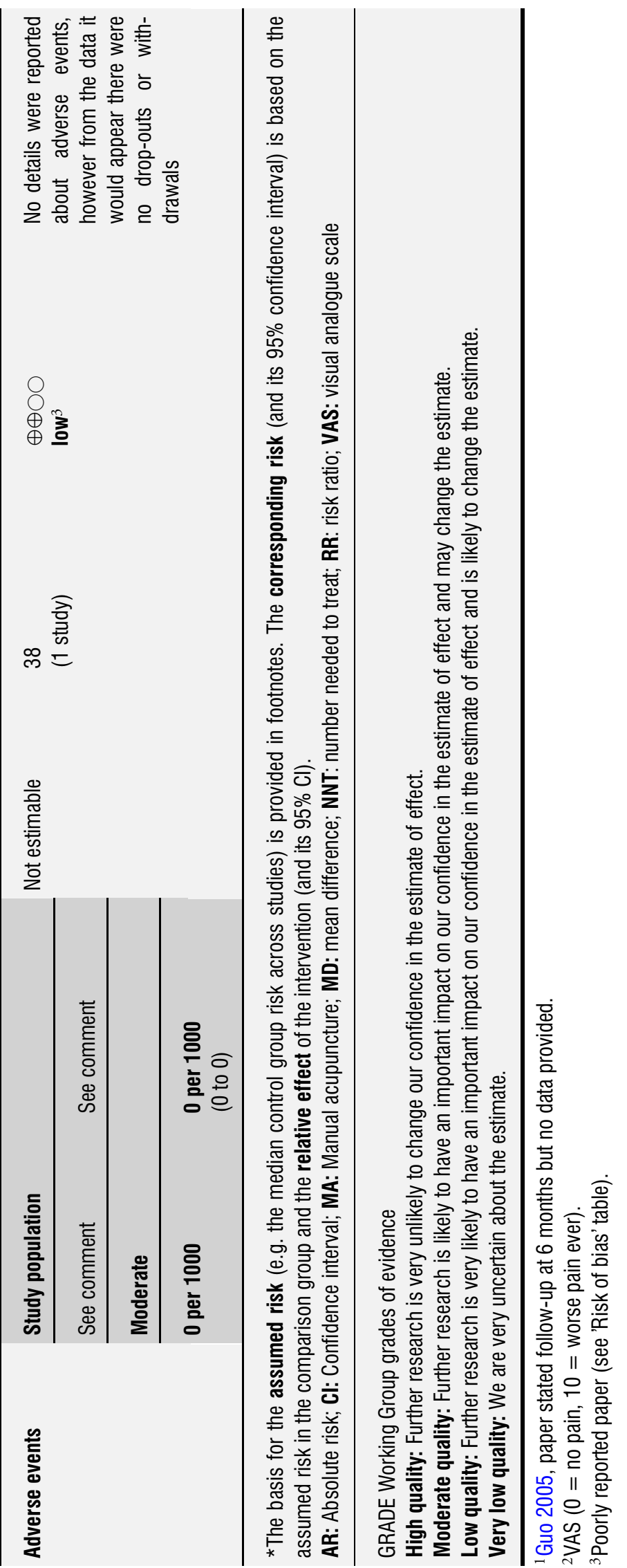

Acupuncture for treating fibromyalgia (Review)

Copyright $\odot 2013$ The Cochrane Collaboration. Published by John Wiley \& Sons, Ltd. 


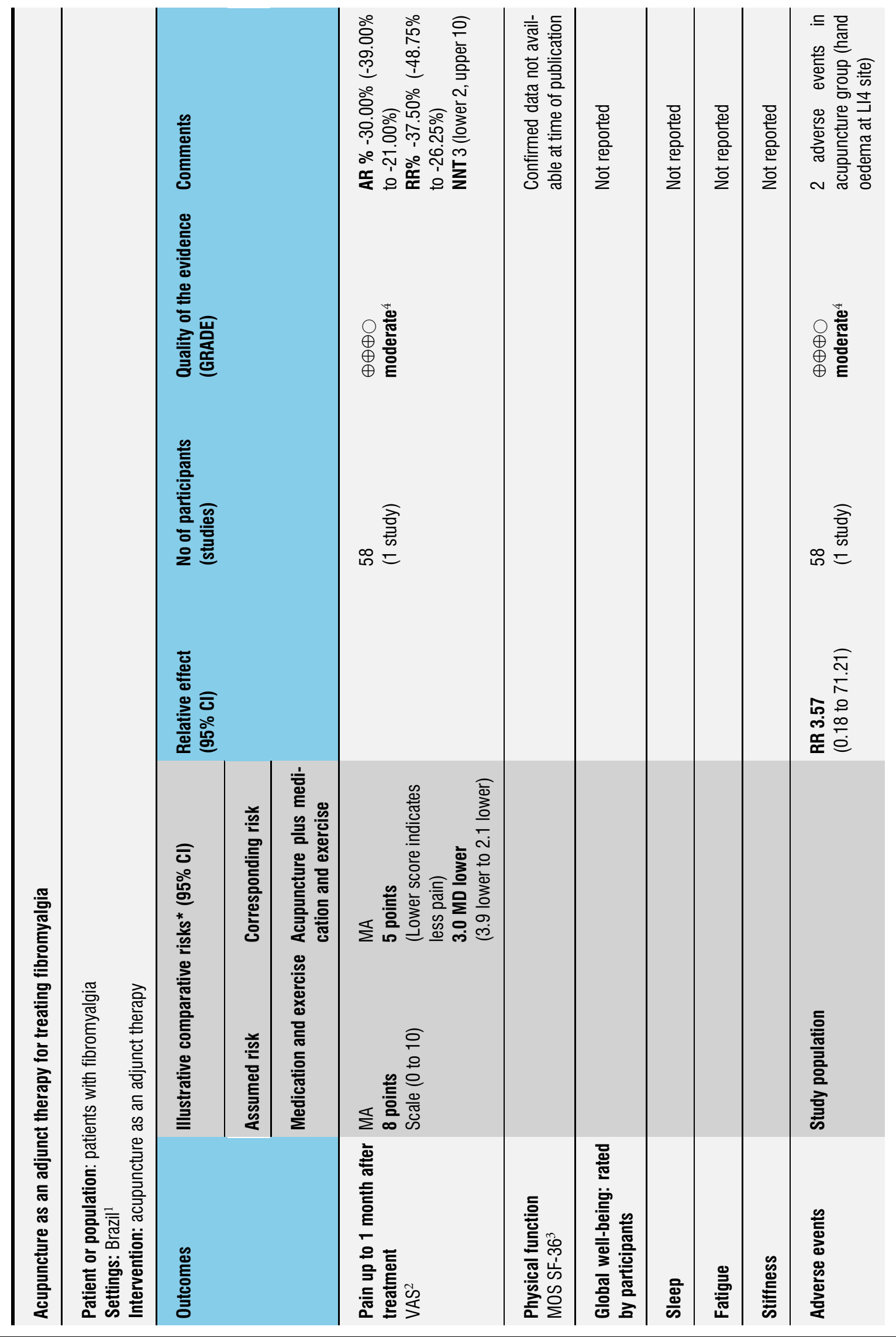




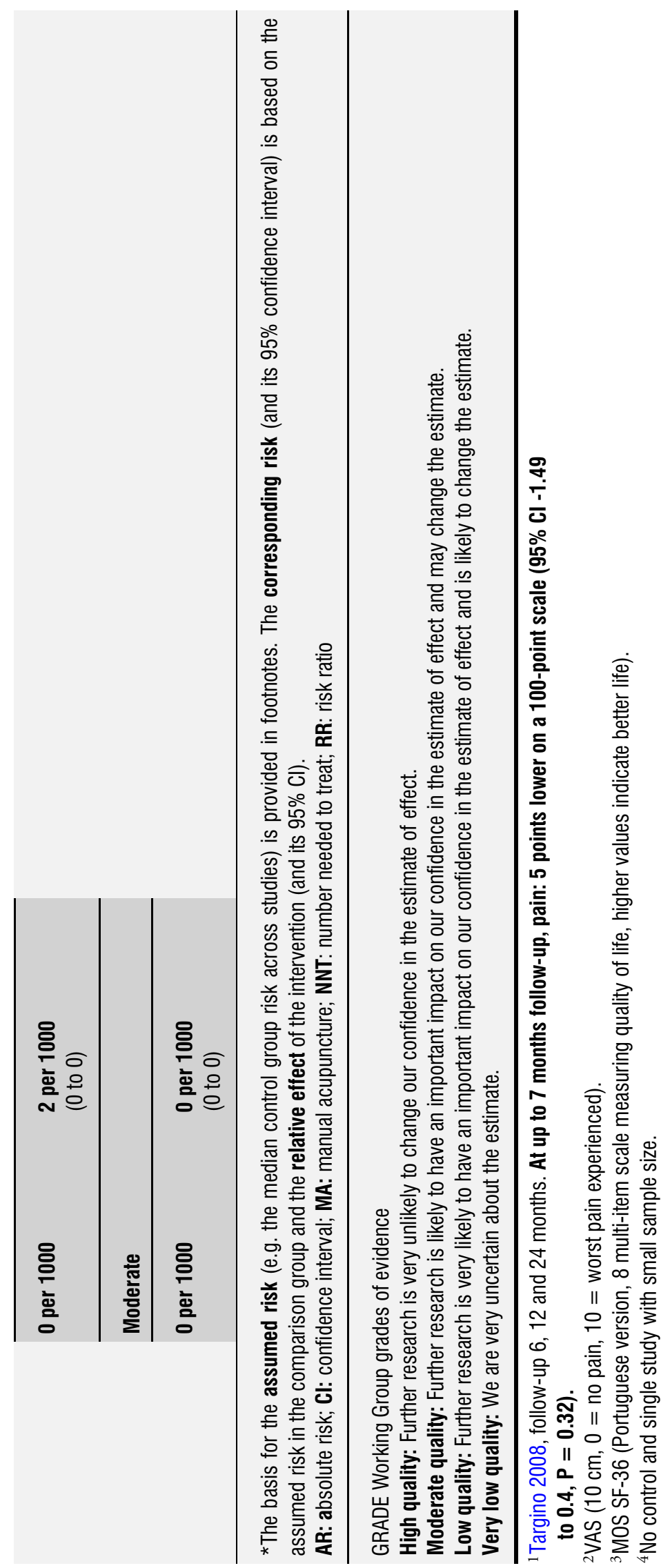

Acupuncture for treating fibromyalgia (Review)

Copyright @ 2013 The Cochrane Collaboration. Published by John Wiley \& Sons, Ltd. 


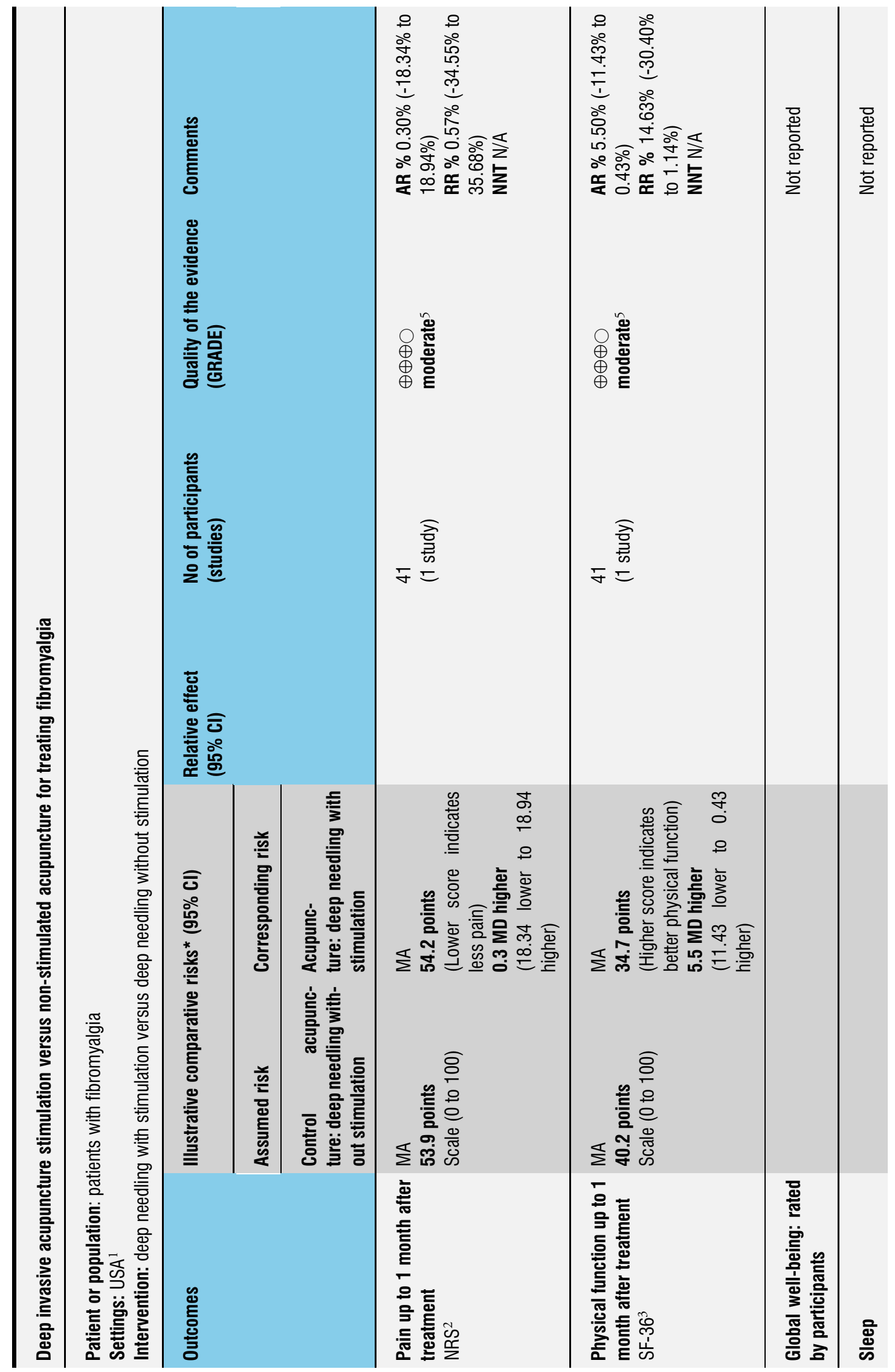




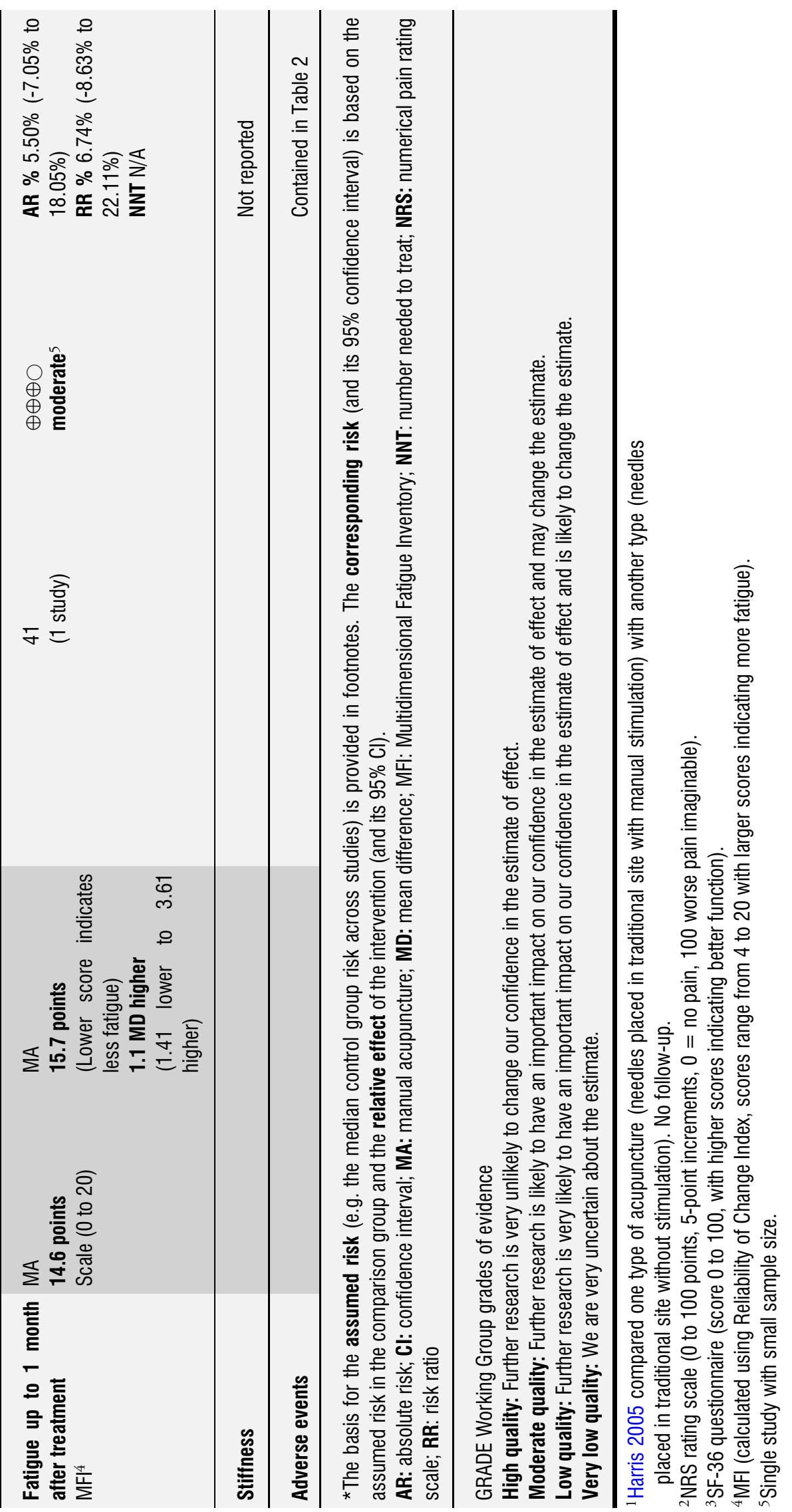

Acupuncture for treating fibromyalgia (Review)

Copyright $\subset 2013$ The Cochrane Collaboration. Published by John Wiley \& Sons, Ltd. 


\section{DISCUSSION}

\section{Summary of main findings}

Out of 124 studies screened, we identified nine randomised controlled trials (RCTs) involving 395 participants. Most of the studies were excluded because of insufficient data. All selected studies used a fixed set of acupuncture points (formula acupuncture) with six using manual acupuncture and three electro-acupuncture. When compared with the group not receiving acupuncture, the acupuncture treatment group improved in terms of pain, global well-being, fatigue and stiffness, but not sleep. We found no difference between real and sham acupuncture on any outcome measures except for stiffness and physical functioning. Stiffness was measured in two electro-acupuncture studies, which showed a moderate effect of electro-acupuncture over sham interventions. On the contrary, sham intervention produced better improvement in physical functioning. Subgroup analyses demonstrated that electro-acupuncture was consistently better than manual acupuncture in eliciting moderate benefits on pain, fatigue, sleep and global well-being as rated by participants.

Comparing

acupuncture with standard pharmacotherapy (amitriptyline), the result of a single trial favoured acupuncture for pain and muscle tenderness. The quality of that paper was poor, affecting the validity of the result. One study examined acupuncture as an adjunct therapy to standard care comprising of a tricyclic antidepressant and exercise and found an additive effect of acupuncture for pain relief and reduction of muscle tenderness.

Measurement of treatment effects was within one month of the end of treatment. Many effects of acupuncture were short-lasting and not maintained at six to seven-month follow-ups. Adverse events reported were mild and no difference between real and sham acupuncture, or other control interventions, was found.

Overall, there is a low to moderate level of evidence indicating that formula acupuncture could be a safe option for fibromyalgia. There is a low to moderate level of evidence that acupuncture is better than non-acupuncture, Western medication and standard therapy in improving pain and stiffness for people with fibromyalgia. There is a moderate level of evidence that the effect of acupuncture does not differ from sham acupuncture in terms of reduction of pain, fatigue, improvement of sleep or global well-being. Subgroup analyses indicate that electro-acupuncture was consistently better than sham interventions. When considering acupuncture, electro-acupuncture could be an effective modality for short-term pain relief. We reached these gradings because of the small sample sizes in all included studies, although the risks of biases were low. None of the studies had more than 50 participants in any of the trial arms and there is a possibility of random errors due to small sample size. As a result, our findings warrant further research with an adequate sample size and long-term follow-up.

\section{Quality of the evidence}

With additional information provided by the authors, we were able to ascertain that the risk of bias of the included studies was acceptable in all studies except for three pragmatic trials (Guo 2005; Itoh 2010; Targino 2002). The other six studies (Assefi 2005; Deluze 1992; Harris 2005; Harris 2008; Harris 2009; Martin 2006) that compared acupuncture with sham controls included acupuncturenaive participants, adopted adequate randomisation procedures, blinded assessors, properly recorded drop-outs and five out of six studies used intention-to-treat analysis. Five studies also blinded participants (Assefi 2005; Harris 2005; Harris 2008; Harris 2009; Martin 2006), tested the blinding of participants (except for Harris 2008) and reported that the participants could not tell to which group they were allocated. We consider that the risk of bias is low in these six out of the nine included studies.

\section{Acupuncture versus non-acupuncture}

There is low-quality evidence based on one trial (electro-acupuncture, 13 participants) that acupuncture significantly reduced pain and stiffness and improved global well-being and fatigue when compared with the non-acupuncture group. We downgraded the quality of evidence because participant blinding was impossible, intention-to-treat analysis was not used and due to the small sample size. Adverse events were not reported. Three participants withdrew due to ineffective treatment (Summary of findings for the main comparison).

\section{Acupuncture versus sham acupuncture}

There is moderate-quality evidence based on data from six trials (289 participants) (combined manual and electro-acupuncture) that acupuncture did not significantly reduce pain compared with sham acupuncture, but subgroup analysis indicates that electro-acupuncture was significantly better than sham electroacupuncture; whereas manual acupuncture showed no significant difference from sham manual acupuncture. Based on one manual acupuncture trial (56 participants), there is moderate-quality evidence that sham acupuncture improved physical function better than acupuncture, with the quality being downgraded due to inconsistency with other outcome measures. Global well-being has moderate-quality evidence based on three trials (203 participants, combined manual and electro-acupuncture) that acupuncture was not better than sham acupuncture, with subgroup analysis indicating that electro-acupuncture showed greater improvement than manual acupuncture did. Sleep had moderate-quality evidence with data from three trials (203 participants) that acupuncture (combined electro- and manual) did not significantly improve sleep time over sham acupuncture. However, subgroup analysis indicates that electro-acupuncture improved sleep quality significantly. We downgraded the quality of evidence for 'pain', 'global well-being' and 'sleep' due to one study (Deluze 1992) not using intention-to-treat analysis. There is high-quality evidence 
based on three trials (204 participants, combined manual and electro-acupuncture) that acupuncture did not significantly reduce fatigue, however subgroup analysis indicates that electro-acupuncture reduced fatigue significantly. Stiffness has moderate-quality evidence based on two trials (104 participants, electro-acupuncture only) that acupuncture significantly reduced stiffness compared with sham acupuncture and was downgraded due to one study (Deluze 1992) not using intention-to-treat analysis. Moderate-quality evidence from six trials (289 participants) showed no statistically significant difference between real and sham acupuncture in the number of adverse events associated with acupuncture. We downgraded the quality due to the small sample size within the studies. One in six people who had acupuncture reported adverse events, in contrast to one in three in the sham treatment groups. Such events were minor and lasted less than one day (Summary of findings 2).

\section{Acupuncture versus medication}

There is low-quality evidence based on one trial (38 participants, manual acupuncture only) that acupuncture significantly reduced pain when compared with medication. We downgraded the quality of evidence due to the poor reporting of the paper. No details about adverse events were reported. From the data it would appear that there were no drop-outs or withdrawals (Summary of findings $3)$.

\section{Acupuncture as an adjunct therapy}

There is moderate-quality evidence based on one trial (manual acupuncture, 58 participants) that acupuncture significantly reduced pain as an adjunct therapy to medication and exercise. We downgraded the quality of evidence due to the small sample size. There were two adverse events in the acupuncture group, which was not significantly different from the control group. We downgraded the evidence due to small sample size (Summary of findings 4).

\section{Deep needling with stimulation versus deep needling without stimulation}

There is moderate-quality evidence based on one trial (manual acupuncture, 41 participants) which showed that there was no significant difference between the two needling styles in the reduction of pain or improvement of their physical function. We downgraded the evidence due to small sample size (Summary of findings 5).

\section{Comparison with other systematic reviews}

Three meta-analyses of RCTs of acupuncture for the treatment of fibromyalgia have recently been published (Cao 2010; Langhorst 2010; Martin-Sanchez 2009) with conflicting conclusions. Cao
2010 considered that acupuncture could be a safe and effective therapy for treating fibromyalgia, while Martin-Sanchez 2009 and Langhorst 2010 concluded that acupuncture was neither effective nor could the effect be distinguished from bias.

In comparison, the present review has the following strengths: our search was comprehensive, including both English and Chinese databases; we adopted strict trial selection criteria based on the American College of Rheumatology (ACR) requirements; the adequacy of acupuncture protocol and treatment delivery were assessed by experts in the field; we contacted all authors to obtain additional data; we extracted data for multiple outcome measures and we limited acupuncture intervention to needling only. Laser acupuncture differs from manual or electro-acupuncture due to its mechanism and depth of stimulation. We selected studies using invasive needling acupuncture as the main or adjunct therapy. Consequently, all studies identified for inclusion in those three reviews have been either included in or excluded from our review. Martin-Sanchez 2009 only examined pain and did not include other outcome measures that are associated with fibromyalgia. Langhorst 2010 included most of the studies selected for this review. Langhorst 2010 found the reduction of pain to be significantly better in the real acupuncture group post-treatment (standardised mean difference (SMD) $-0.25 ; 95 \%$ confidence interval (CI) -0.49 to $-0.02, \mathrm{P}=0.04$ ) and their effect size was smaller when compared with our data (SMD -0.42) due to inclusion of two studies that we excluded (Lautenschlager 1989; Sprott 1998) for not using ACR criteria or not reporting confirmable data, respectively. They also excluded one study which was included in our review (Harris 2008). Langhorst 2010 went on to conclude that "significant reduction of pain was only present in studies with risk of bias" because they considered three positive studies (Assefi 2005; Deluze 1992; Martin 2006) as having a high risk of bias. As indicated in Figure 3 and Figure 2, our data do not support this claim as explained above in the 'Quality of the evidence' section. We also included three other studies comparing acupuncture with non-acupuncture, medication and standard therapy.

\section{Outcome measures for fibromyalgia}

The top three core domains for outcome measures in any pain studies as recommended in IMMPACT are pain, function and emotion (Dworkin 2010). Furthermore, the 2010 ACR preliminary diagnostic criteria identified pain as well as a range of nonpain symptoms, for instance cognitive symptoms, headache and irritable bowel syndrome (Wolfe 2010). In all studies included in this review, the measurement tools for pain were adequate and validated. However, only four studies measured function or quality of life, two studies measured mental well-being, with one using the depression and anxiety sub-scales of the Fibromyalgia Impact Questionnaire (FIQ) and the other using the mental component of the SF-36. None measured cognition or somatic symptoms apart from sleep and fatigue. The FIQ, a condition-specific, validated 
function measure, was used only in two studies (Itoh 2010; Martin 2006). The FIQ has been in existence for 18 years and translated into eight languages (Bennett 2005). Targino 2008 explained they could not use the FIQ because the Brazilian version had not been validated at the time of the trial. The other two (Assefi 2005; Harris 2005) used the SF-36, which assesses quality of life but not function in fibromyalgia. The data from Assefi 2005 could not be used for the current review due to incorrect labelling of data in the published papers. To our knowledge, there is only one trial comparing the FIQ and the SF-36 in fibromyalgia and rheumatoid arthritis participants (Birtane 2006). The total score on the FIQ was moderately correlated with physical function, physical role and bodily pain on the SF-36, but not with other domains. Sub-scales of the FIQ were not correlated with relevant domains on the SF-36. For instance, bodily pain on the SF-36 was correlated with stiffness but not pain on the FIQ, and mental health on the SF-36 was correlated with anxiety but not depression on the FIQ. For this reason, we analysed data from the SF-36 and the FIQ separately, with the SF-36 measuring physical and mental function and the FIQ measuring overall well-being. We question the suitability of the SF-36 for measuring function in fibromyalgia participants. Physical function measured with the SF-36 physical domain was poorer in the acupuncture group than in the sham intervention group. The change was statistically, but not clinically, significant. We could not explain this finding. Given that the finding was from one trial, future studies with large sample sizes might impact on the direction of changes.

Fibromyalgia is characterised by widespread chronic pain as well as a range of non-pain symptoms and co-morbidities. A recent review indicates that when rating global improvement, fibromyalgia participants consider not only pain reduction, but also improvement in fatigue, functioning, mood and daily living (Hudson 2009). It is therefore important to assess a wide range of measures when examining the effect of any interventions for fibromyalgia. The FIQ consists of measures of pain, fatigue, sleep and physical and emotional functioning and is an ideal outcome measurement tool. OMERACT (Outcome Measures in Rheumatology) participants have agreed that pain, tenderness, fatigue, participant global rating or well-being, function and sleep are the core outcomes to be measured (Mease 2009). The FIQ measures most of these domains. In future studies, researchers should consider using the FIQ or include the assessment of the key co-morbidities and emotional and cognitive aspects of fibromyalgia. Such a design would help identify the specific effects of acupuncture on fibromyalgia.

\section{Quality of acupuncture treatment}

Overall, the treatment was adequate in terms of frequency (two to three sessions per week), number of treatments (six to 28 sessions) and length of each session of treatment (20 to 30 minutes). However, reporting of some details of the treatment, such as needling depth and unilateral or bilateral needling, were unavailable. It is important that both authors and journals adhere to the STRICTA guidelines for adequate reporting of acupuncture treatments. The major weakness of reporting of acupuncture treatments was a lack of rationale for the acupuncture treatment in all but one included trial (Deluze 1992), and justification of point selection was rarely provided, which could be due to a lack of standard Chinese medicine syndrome differential criteria for fibromyalgia. Acupuncture therapy in a clinical setting relies on the syndrome pattern differentiation for accurate point selection. None of the studies included offered a diagnosis or attempted a syndrome pattern differentiation for fibromyalgia according to Chinese medicine.

The current Western medicine diagnosis of fibromyalgia does not result in a single entity or homogenous group. Reliance on the two main criteria of chronic widespread pain and 11 out of 18 tender points according to the ACR diagnostic criteria has been criticised for not considering other important symptoms and comorbidities (Mease 2005; Wilke 2009; Wolfe 2003). It was never intended for the ACR criteria to be used for clinical diagnosis but rather for research as a standardised definition of fibromyalgia, and there is no gold standard for fibromyalgia diagnosis (Katz 2005). To address this, Western medical research is being undertaken to examine the differentiation of fibromyalgia into subgroup/symptom clusters (Muller 2007; Schneider 2005; Wilson 2009). The 2010 ARC preliminary criteria (Wolfe 2010) are a positive step towards clinically orientated approaches.

Although fibromyalgia is not a diagnosis of Chinese medicine, the types of pain and co-morbidities associated with it may fit into the Chinese medicine diagnostic concept of Bi-Syndrome, documented 2500 years ago (Ni 1995). Dividing Bi-Syndrome into a number of patterns depends on the characteristics of pain, as well as the accompanying signs and symptoms, which allows syndrome pattern differentiation, leading to an individualised approach to treatment that is part of the clinical decision-making process within traditional/clinical acupuncture practice. However, the Chinese medicine diagnostic criteria for fibromyalgia are yet to be developed. This might explain why only formula acupuncture treatments were used in all included studies. It is common in modern Chinese medicine for a disease or condition in Western medicine to be given a set of differentiation diagnoses so that understanding of subgroups can be standardised to provide guidance for treatment. There is an urgent need for research into developing Chinese medicine syndrome differentiation diagnostic criteria.

\section{Modes of acupuncture}

The current data do not allow us to conclude the best acupuncture stimulation mode for the treatment of fibromyalgia. However, only one trial examined the two types of stimulation, and found deep needling with stimulation did not differ from deep needling without stimulation. That is to say deqi, one of the essences of acupuncture stimulation, might not play the expected role in the 
treatment of fibromyalgia. Subgroup analyses indicate that electroacupuncture was consistently superior to manual acupuncture for a number of major outcome measures. However, no trial directly compared electro- with manual acupuncture.

Sensitivity of the nervous system of fibromyalgia participants may influence the treatment outcome of different types of stimulation, however dose of treatment could be another explanation. A recent trial in healthy humans compared the effect of sham acupuncture with manual and electro-acupuncture on electrical pain thresholds (Zheng 2010). The researchers found that electro-acupuncture induced the best analgesia, followed by manual acupuncture, then the sham intervention. In electro-acupuncture, the stimulation was delivered constantly for 25 minutes, whereas in manual acupuncture the stimulation was about one minute and in sham acupuncture was close to zero. The treatments were similar to those in the selected studies for this review. That is, any difference between electro- and manual or manual with sham manual acupuncture could be due to the duration and strength of stimulation, or dose. This hypothesis will need to be tested in a trial examining all three modes of stimulation in the fibromyalgia population.

\section{Challenges of sham acupuncture design in fibromyalgia studies}

Sham acupuncture controls varied amongst the studies. We conducted a subgroup analysis comparing studies using invasive sham acupuncture with studies using a non-invasive method and found no subgroup difference. This comparison is, however, influenced by the small number of studies and mixed studies using electroand manual acupuncture. Consequently, we could not draw a strong inference as to what the ideal sham control is. Sham controls were non-invasive (Assefi 2005 (one arm) and Harris 2008; Harris 2009; Martin 2006), invasive, off the point/channel (Assefi 2005 (one arm); Deluze 1992; Harris 2005 (two arms)) plus invasive on irrelevant point (Assefi 2005 (one arm)). The inert nature of these sham methods is debatable and no agreed standard for sham controls exists for acupuncture (Birch 2006). Penetrating the skin anywhere would appear to activate one of the commonly proposed mechanisms of acupuncture analgesia, i.e. diffuse noxious inhibitory control (Lewith 1983; Pomeranz 1988). Four of the included studies reported that their choice of sham might in fact be active (Assefi 2005; Harris 2005; Martin 2006; Targino 2008). Indeed, in a trial comparing muscle blood flow in fibromyalgia participants with that of healthy controls, the researchers found that in healthy controls only deep needle insertion into an acupuncture point increased blood flow, but in fibromyalgia participants both shallow and deep insertion were equally effective (Sandberg 2004).

In a review, Lundeberg 2007 questioned whether sham acupuncture was a valid procedure for fibromyalgia participants due to their dysfunctional central nervous system (central sensitisation). As such, the nervous system may be responsive to the sub-pain threshold stimulus involved in any invasive sham acupuncture, subsequently activating the endogenous pain inhibition pathways (Mense 2003) that are usually activated by painful stimulation. Further clouding the issue is the result of a positron emission tomography (PET) trial of participants with fibromyalgia (Harris 2009). They found no difference between real manual acupuncture and non-invasive sham manual acupuncture in pain reduction. However, they identified significant group difference in brain activities. Morphine binding potential was increased in the real manual acupuncture group in the brain centres that modulated pain, whereas it was reduced or there was no change in the non-invasive sham manual acupuncture group. The results indicate that a non-invasive sham acupuncture technique may become active treatment in this population group, and its mechanism is likely due to non-opioid mediated pain modulation. This might also explain why there was no difference between deep needling with and without stimulation.

The placebo effect, including a range of components such as patient expectation, patient/therapist relationship and conditioning, has also been considered as one of the mechanisms explaining acupuncture analgesia (Finniss 2010). An analysis of data from four acupuncture trials totaling 864 participants concluded that there is a strong association between expectation and pain relief (Linde 2007). In a qualitative study, Kerr and colleagues (Kerr 2011) found that trial participants interpreted the sensation elicited by non-invasive placebo acupuncture needles as being meaningful and therapeutic. Those studies indicate that acupuncture is a complex intervention with multiple components. Indeed, some researchers challenge the usefulness of sham acupuncture controlled trials (Langevin 2011).

However, having some form of placebo is important in establishing the efficacy of a therapy. Future studies need to identify an adequate sham acupuncture intervention for fibromyalgia participants before studies are commenced. It is also important to conduct highquality pragmatic trials to compare acupuncture with other proven therapies. In the current review, we found that acupuncture was superior to antidepressants and a combination of antidepressants and exercise for fibromyalgia, but the findings were from two studies with a small sample size.

\section{Reporting of adverse events}

We identified inconsistent reporting of adverse events in the included studies, with some studies reporting no adverse events (Harris 2005; Harris 2008) and others reporting 53\% of participants experiencing them (Assefi 2005). So far, there is no uniform understanding of what constitutes an adverse event in acupuncture treatment or what should be recorded. For example, should 'pain at site of needling' be an adverse event, when for some techniques this is normal? Is being 'relaxed/tired' an adverse event or a typical indication of the therapeutic effect of acupuncture? 
The discrepancy of reporting adverse events is also reflected by other published studies. For example, one clinic audit found that bleeding occurring in 53\% cases and pain in 24\% (White 2001), whereas another reported bleeding in $0.4 \%$ and pain in $1.2 \%$ of cases (MacPerson 2001). Although the former study audited treatments performed by medical or physiotherapy acupuncturists and the latter by traditional Chinese acupuncturists, the significant differences are likely due to reporting discrepancy and the definition of adverse events. Generally, acupuncture is considered safe. A consensus on how to report adverse events in acupuncture treatment is needed.

\section{AUTHORS'CONCLUSIONS}

\section{Implications for practice}

Due to the weaknesses of the included studies, the implications for practice are limited. Overall, there is a low to moderate-quality level of evidence that formula acupuncture for the treatment of fibromyalgia is safe. There is a moderate level of evidence that acupuncture is not better than sham controls. Electro-acupuncture is found to be consistently better than sham interventions in improving pain, global well-being, sleep, stiffness and fatigue. The effect of acupuncture was not maintained at six to seven months after treatment. The same level of evidence supports acupuncture as an adjunct therapy to medication and exercise or acupuncture when compared with a medication and exercise control. When comparing acupuncture with medication or a wait list, there is low quality evidence in favour of acupuncture but this needs more rigorous and methodologically sound studies.

Evidence suggests that treatment sessions should be twice per week, over four weeks, with each session lasting for 25 minutes. Electroacupuncture seems to provide a number of benefits for fibromyalgia participants. Practitioners should consider electro-acupuncture with 2 to $5 \mathrm{~Hz}$ electrical stimulation and acupuncture points could include ST36 and LI4. Optimal needling depth, point selection and needle stimulation are yet to be identified.

Like any treatment for chronic pain, maintenance acupuncture treatment is likely to be required for long-term benefit for fibromyalgia. How frequent the treatment should be is unknown.

\section{Implications for research}

We recommend a number of ways in which to address the weaknesses identified in the included studies. To further test the usefulness of acupuncture in treating fibromyalgia, researchers need to develop Chinese medicine diagnostic and subgroup differentiation criteria. The suitability of any sham acupuncture needs to be tested in this population prior to any further studies. In regards to the safety profile, a clear definition of what adverse events are associated with acupuncture is needed. Future studies testing the efficacy of acupuncture should use an adequate sample size, apply electroacupuncture and assess the long-term results. Use of a diseasespecific tool, such as the Fibromyalgia Impact Questionnaire, and accurate reporting of treatment using the Standards for Reporting Interventions in Controlled studies of Acupuncture (STRICTA) guidelines would be desirable. Future studies also need to assess how often acupuncture should be delivered to maintain its longterm benefit and the cost-effectiveness of such a treatment plan.

\section{ACKNOWLEDGEMENTS}

We would like to thank Robin Christensen at CMSG for statistical support and advice; Anette Bluemie at the German Cochrane Centre, Lorenzo Moja at the Italian Cochrane Centre and Juan Molero at the School of Health Sciences, RMIT University, Australia, for translating potential papers; and the authors of Targino 2008, Harris 2007 (excluded), Harris 2008 and Itoh 2010 for providing papers and raw data directly to us.

\section{REFER E N C E S}

\section{References to studies included in this review}

Assefi 2005 \{published and unpublished data\}

* Assefi NP, Sherman KJ, Jacobsen C, Goldberg J, Smith WR, Buchwald D. A randomised clinical trial of acupuncture compared with sham acupuncture in fibromyalgia. Annals of Internal Medicine 2005;143(1): $10-9$.

Deluze 1992 \{published data only\}

* Deluze C, Bosia L, Zirbs A, Chantraine A, Visher TL. Electroacupuncture in fibromyalgia: results of a controlled trial. BMJ 1992;305:1249-52.
Guo 2005 \{published data only\}

* Guo Y, Sun YZ. Clinical study on treatment of fibromyalgia syndrome with penetration needling at back. Chinese Acupuncture \& Moxibustion 2005;25(2):98-100.

Harris 2005 \{published and unpublished data\}

* Harris RE, Tian X, Williams DA, Tian TX, Cupps TR, Petzke F, et al.Treatment of fibromyalgia with formula acupuncture: investigation of needle placement, needle stimulation and treatment frequency. Journal of Alternative and Complementary Medicine 2005;11(4):663-71.

Harris 2008 \{published and unpublished data\} Harris RE, Sundgren PC, Pang Y, Hsu M, Petrou M, Kim 
$\mathrm{SH}$, et al.Dynamic levels of glutamate within the insula are associated with improvements in multiple pain domains in fibromyalgia. Arthritis \& Rheumatism 2008;58(3):903-7.

Harris 2009 \{published and unpublished data\}

* Harris RE, Zubieta JK, Scott DJ, Napadow V. Traditional Chinese acupuncture and placebo (sham) acupuncture are differentiated by their effects on $\mu$-opioid receptors (MORs). NeuroImage 2009;47:1077-85. [: Acupuncture vs sham acupuncture]

Itoh 2010 \{published and unpublished data\}

${ }^{*}$ Itoh K, Kitakoji H. Effects of acupuncture to treat fibromyalgia: a preliminary randomised controlled trial. Chinese Medicine 2010;5(11):1-7.

Martin 2006 \{published and unpublished data\} * Martin DP, Sletten CD, Williams BA, Berger IH. Improvements in fibromyalgia symptoms with acupuncture: results of a randomised controlled trial. Mayo Clinic Proceedings 2006;81(6):749-57.

Targino 2008 \{published and unpublished data\} Targino RA, Imamura M, Kaziyama HHS, Souza PM, Hsing WT, Furian AD, et al.A randomised controlled trial of acupuncture added to usual treatment for fibromyalgia. Journal of Rehabilitation Medicine 2008;40:582-8.

\section{References to studies excluded from this review}

\section{Cao 2003 \{published data only\}}

Cao JQ, Li Y. The combined therapy of anti-depressants and acupuncture-moxibustion for fibromyalgia syndrome-a report of 56 cases. Journal of Chinese Medicine and Drugs 2003;21(5):813-7.

Cassisi 1994 \{published data only (unpublished sought but not used)\} Cassisi G, Roncaglione A, Ceccherelli F, Donolato C, Gagiardi G, Todesco S. Acupuncture treatment for primary fibromyalgia. Confronted with Mianserina [Trattamento agopunturale della fibromialgia primaria. Confronto con Mianserina]. Giornale Italiano Di Riflessoterapia 1994;6(23):5-9.

Cassisi 1995 \{published data only (unpublished sought but not used)\} Cassisi G, Roncaglione A, Ceccherelli F, Donolato C, Gagliardi G, Todesco S. Acupuncture treatment for primary fibromyalgia. Confronted with Mianserina [Trattamento agopunturale della fibromialgia primaria. Confronto con Mianserina]. Giornale Italiano Di Riflessoterapia 1995;7(1): 33-6.

Chen 2009 \{published and unpublished data\}

Chen ZL. Acupuncture treatment of 32 cases of fibromyalgia syndrome. World Health Digest Medical Periodical 2009;6 (24):270-1.

Collazo Chao 2010 \{published data only\}

* Collazo Chao E. Effectiveness of acupuncture therapy for pain relief in patients with fibromyalgia. Revista Internacional de Acupuntura 2010;4(1):52-8.

Dai 2009 \{published data only\}

Dai JZ. Combined therapy of Chinese herbal medicine for primary fibromyalgia: clinical observation of 396 cases.
Journal of Beijing University of Traditional Chinese Medicine 2009;32(4):278-9.

Feldman 2001 \{published data only (unpublished sought but not used)\} Feldman D, Mariano E. Treatment of fibromyalgia with acupuncture: a randomised, placebo controlled trial of 16 weeks duration. American College of Rheumatology Meeting. 2001:Abstract 91.

Gong 2010 \{published data only\} Gong WZ, Wang YQ. Observation on the therapeutic effect of acupuncture on fibromyalgia syndrome. Shanghai Journal of Acupuncture and Moxibustion 2010;29(11):725-7.

Gou 2010 \{published data only\}

* Gou AS, Li AH. The effect of electroacupuncture combined with TDP treatment for 36 cases of fibromyalgia syndrome. Journal of Communication 2010;24(4):410-1.

Guan 2005 \{published data only\} Guan BS, Li J. The combined therapy of acupuncture and cupping for fibromyalgia syndrome - a report of 28 cases. Chinese Journal of Gerontology 2005;25:826-7.

Guevara 2007 \{published data only (unpublished sought but not used)\} Guevara M, Scott D, Zubieta J, Clauw D, Harris R. Relationship between expectation and $\mu$-opioid receptor (MOR) blinding prior acupuncture and sham acupuncture treatment in fibromyalgia. Journal of Pain 2007;8(4): A1-14.

Guo 2003 \{published data only\} Guo XJ, Jia J. Comparison of therapeutic effects transcutaneous electrical nerve stimulation and electroacupuncture on fibromyalgia syndrome. Chinese Acupuncture \& Moxibustion 2003;23(11):653-5.

\section{Guo 2005a \{published data only\}}

Guo X, Jia J. Comparison of therapeutic effects on fibromyalgia syndrome between dermal-neurological electric stimulation and electric acupuncture. Chinese Journal of Clinical Rehabilitation. 2005;9(46):171-3.

Harris 2006 \{published data only\} Harris RE, Gracely RH, McLean SA, Williams DA, Giesecke T, Petzke F, et al.Comparison of clinical and evoked pain measures in fibromyalgia. Journal of Pain 2006; 7:521-7.

Harris 2007 \{published data only\} Harris RE, Clauw DJ, Scott DJ, McLean SA, Gracely RH, Zubieta JK. Decreased central $\mu$-opioid receptor availability in fibromyalgia. Journal of Neuroscience 2007;27(37): 10000-6.

Harris 2007a \{published data only\} Harris RE, Zubieta JK, Scott DJ, Gracely RH, Clauw DJ. Differential sustained changes in $\mu$-opioid receptor (MOR) availability following acupuncture and sham acupuncture therapy in fibromyalgia. Journal of Pain 2007;8(4):A1-14.

Harris 2007b \{published data only\} Harris RE, Scott D, Guevara M, Gracely R, Zubieta J, Clauw D. mu-Opioid receptor (MOR) binding predicts differential responsiveness to acupuncture and sham 
acupuncture therapy in fibromyalgia. Journal of Pain 2007; 8(4):A1-14.

Jiang 2010 \{published data only\} Jiang ZY, Li CD, Xiou L, Guo JH, He LN, Yue Y, et al.Combination of acupuncture, cupping and medicine for treatment of fibromyalgia syndrome: a multi-central randomised controlled trial. Chinese Acupuncture and Moxibustion 2010;30(4):265-9.

Lautenschlager 1989 \{published data only\} Lautenschlager J, Schnorrenberger CC, Muller W. Acupuncture for generalised Tendomyopathie (fibromyalgia syndrome) [Akupunktur bei generalisierter Tendomyopathie (fibromyalgie-syndrom)]. Deutsche Zeitschrift Fur Akupunktur 1989;32:122-8.

Li 2005 \{published data only\} Li A, Wang ZL, Ping Y. Clinical observation of the treatment of primary fibrositis syndrome by Ding Tongtang combined with acupuncture. Chinese Journal of Current Clinical Medicine 2004;2(9A):1387-8.

Li 2005a \{published data only\}

Li J, Yu HB, Yu Z. The clinical effect of acupuncture and computer intermediate frequency for fibromyalgia syndrome. Chinese Journal of Modern Medicine and Drugs 2005;7(1):42-4.

Li 2006 \{published data only\} Li CD, Fu XY, Jiang ZY, Yang XG, Huang SQ, Wang, et al.Clinical studies on combination of acupuncture, cupping and western medicine for treatment of fibromyalgia syndrome. Chinese Acupuncture \& Moxibustion 2006;26(1): $8-10$.

Li 2008 \{published data only\}

Li FJ. Shu-mu combination for the treatment of 26 cases of fibromyalgia. Information on Traditional Chinese Medicine 2008;25(5):75-6.

Li 2010 \{published data only\}

Li R, Guo LG. The nursing management for fibromyalgia syndrome. West China Medical Journal 2010;25(3):644.

Lui 2002 \{published data only\}

Lui Q, Li F. Clinical observation of acupuncture for 30 cases of fibromyalgia. Anthol Med 2002;21:183-4.

Sandberg 1999 \{published data only\}

Sandberg M, Lundeberg T, Gerdle B. Manual acupuncture in fibromyalgia: long term pilot study. Journal of Musculoskeletal Pain 1999;7(3):39-58.

Sandberg 2004 \{published data only\} Sandberg M, Lindberg LG, Gerdle B. Peripheral effects of needle stimulation (acupuncture) on skin and muscle blood flow in fibromyalgia. European Journal of Pain 2004; 8:163-71.

Sprott 1995 \{published data only (unpublished sought but not used)\} Sprott H. [Schmerzbehandlung der generalisierten tendomyopathie (GTM, fibromyalgie) durch akupuntur]. Natura Medicine 1995;10:43-9.
Sprott 1998 \{published data only\}

Sprott H. Efficiency of acupuncture in patients with fibromyalgia. Clinical Bulletin of Myofascial Therapy 1998;3 (1):37-43.

Sprott 2000 \{published data only\}

Sprott H, Jeschonneck M, Grohmann G, Heln G. Changes in blood flow over tender points of fibromyalgia patients after acupuncture (measured with the laser-doppler flowmeter) [Anderung der Durchblutung uber den tender points bei Fibromyalgie-Patienten nach einer Akupunkturtherapie (gemessen mit der Laser-doppler-flowmetrie)]. Wiener Klinische Wochenschrift 2000;112(13):580-6.

Sun 2008 \{published data only\}

Sun Y. Acupuncture and moxibustion the treatment for 28 cases of fibromyalgia syndrome. World Chinese Medicine 2008;3(3):170-1.

Targino 2002 \{published data only (unpublished sought but not used)\} Targino RA, Imamura M, Kaziyama HHS, Souza LPM, Hsing WT, Imamura ST. Pain treatment with acupuncture for patients with fibromyalgia. Current Pain and Headache Reports 2002;6:379-83.

Uhlemann 2001 \{published data only (unpublished sought but not used)\}

Uhlemann C, Schreiber TU, Smolenski UC, Loth D. [Randomisierte studle zur akupunktur und bindegewebsmassage als therapieoption bei patienten mit fibromyalgiesyndrom (FMS)]. Physikalische Medizin Rehabilitationsmedizin Kurortmedizin. 2001; Vol. 11:153.

Wang 2002 \{published data only\}

Wang SP, Wang XF, Zhang DX, Yang HB. Clinical observation on therapeutic effect of acupuncture treatment based on syndrome differentiation of meridians on fibromyalgia. Chinese Acupuncture \& Moxibustion 2002;22 (12):807-9.

Wang 2004 \{published data only\}

Wang WX, Liu ZT, Wu YC. Clinical observation on acupuncture treatment with differentiation diagnosis for fibromyalgia syndrome: a report of 42 cases. Forum on Traditional Chinese Medicine 2004;19(1):26-7.

Wei 2006 \{published data only\}

Wei S, Qu L, Chen ZH, Xu MC. Point injection of snake venom for fibromyalgia syndrome: a report of 50 cases. Journal of Anhui TCM College 2006;25(6):9-10.

Wu 2003 \{published data only\}

Wu HJ, Fu LP, Wang RH. Clinical observation on acupuncture treatment of 57 patients with fibromyalgia syndrome. Shanghai Journal of Acupuncture and Moxibustion 2003;22(6):15-6.

Yao 2006 \{published data only\}

Yao YP, Zhang XR, Wang X. Acupuncture to regulate DU meridian for treatment of fibromyalgia syndrome. Journal of Clinical Acupuncture and Moxibustion 2006;22(2):24-5.

\section{Zhang 2001 \{published data only\}}

Zhang YG. Clinical observation on acupuncture treatment of primary fibromyalgia syndrome. Chinese Acupuncture \& Moxibustion 2001;21(1):19-20. 
Zhou 2003 \{published data only\}

Zhou ZH, Yu WT, Wu ZH, Wu BX, Dai YY. Clinical observation of ultra-laser therapy on primary fibromyalgia syndrome. China Chinese Medicine for Emergency Medicine 2003;12(6):522, 552.

\section{References to ongoing studies}

Vas 2011 \{published data only\}

Vas J, Modesto M, Aguilar I, Santos-Rey K, Benitez-Parejo N, Rivas-Ruiz F. Effects of acupuncture on patients with fibromyalgia: study protocol of a multi centre randomised controlled trial. Trials 2011;12(59):1-11.

\section{Additional references}

\section{Arnold 2006}

Arnold LM. Biology and therapy of fibromyalgia. New therapies in fibromyalgia. Arthritis Research Therapy 2006;8 (4):212.

Arnold 2011

Arnold LM, Clauw DJ, McCarberg BH, FibroCollaborative. Improving the recognition and diagnosis of fibromyalgia.

Mayo Clinic Proceedings 2011;86(5):457-64.

\section{Bennett 2005}

Bennett R. The Fibromyalgia Impact Questionnaire (FIQ): a review of its development, current version, operating characteristics and uses. Clinical and Experimental Rheumatology 2005;23(Suppl 39):S-154-62.

\section{Bennett 2009}

Bennett RM, Friend R, Jones KD, Ward R, Han B, Ross RL. The revised Fibromyalgia Impact Questionnaire (FIQR): validation and psychometric properties. Arthritis Research and Therapy 2009;11(R120):1-14.

Bergman 2007

Bergman S. Management of musculoskeletal pain. Best Practice \& Research Clinical Rheumatology 2007;21(1): 153-66.

\section{Berman 1999}

Berman BM, Ezzo J, Hadhazy V, Swyers J. Is acupuncture effective in the treatment of fibromyalgia. Journal of Family Practice 1999; 48(3):213-8.

\section{Birch 2006}

Birch S. A review and analysis of placebo treatments, placebo effects, and placebo controls in trials of medical procedures when sham is not inert. Journal of Alternative and Complementary Medicine 2006;12(3):303-10.

Birtane 2006

Birtane M, Uzunca $\mathrm{K}$, Tastekin Tuna $\mathrm{H}$. The evaluation of quality of life in fibromyalgia syndrome: a comparison with rheumatoid arthritis by using SF-36 Health Survey. Clinical Rheumatology 2006;5:679-84.

Bombardier 1996

Bombardier $\mathrm{CH}$, Buchwald D. Chronic fatigue syndrome and fibromyalgia. Disability and health-care use. Medical Care 1996;34(9):924-30.

\section{Boomershine 2009}

Boomershine CS, Crofford LJ. A symptom-based approach to pharmacologic management of fibromyalgia. Nature Reviews Rheumatology 2009;5:191-9.

\section{Burckhardt 1991}

Burckhardt CS, Clark SR, Bennett RM. The Fibromyalgia Impact Questionnaire: development and validation. Journal of Rheumatology 1991;18:728-34.

Burke 2006

Burke A, Upchurch DM, Dye C, Chyu L. Acupuncture use in the United States: findings from the National Health Interview Survey. Journal of Alternative and Complementary Medicine 2006;12(7):639-48.

Cao 2002

Cao X. Scientific bases of acupuncture. Acupuncture Electrotherapeutics Research 2002;27(1):1-14.

Cao 2010

Cao H, Liu JP, Lewith GT. Traditional Chinese Medicine for treatment of fibromyalgia: a systematic review of randomised controlled trials. Journal of Alternative and Complementary Medicine 2010;16(4):397-409.

Dworkin 2010

Dworkin RH, Turk DC, Peirce-Sandner S, Baron R, Bellamy N, Burke LB, et al.Research design considerations for confirmatory chronic pain clinical trials: IMMPACT recommendations. Pain 2010;149(2):177-93.

Ezzo 2000

Ezzo J, Berman B, Hadhazy A, Jadad AR, Lao L, Singh BB. Is acupuncture effective for the treatment of chronic pain? A systematic review. Pain 2000;86(3):217-25.

Finniss 2010

Finniss DG, Kaptghuk TJ, Miller F, Benedetti F. Biological, clinical and ethical advances of placebo effects. The Lancet 2010;375:686-95.

Han 1997

Han J. Acupuncture activates endogenous systems of analgesia. National Institutes of Health Consensus Development Conference on acupuncture. Program and abstracts; 1997 Nov 3-5; Bethesda (MD). 1997:56.

Higgins 2011

Higgins JPT, Green S (editors). Cochrane Handbook for Systematic Reviews of Interventions Version 5.1.0 [updated March 2011]. The Cochrane Collaboration, 2011. Available from www.cochrane-handbook.org.

\section{Hudson 2009}

Hudson JI, Arnold LM, Bradley LA, Choy EH, Mease PJ, Wang $\mathrm{F}$, et al. What makes patients with fibromyalgia feel better? Correlations between Patient Global Impression of Improvement and changes in clinical symptoms and function: a pooled analysis of 4 randomised placebocontrolled trials of duloxetine. Journal of Rheumatology 2009;36(11):2517-22.

Katz 2005

Katz RS, Wolfe F, Michaud K. Fibromyalgia diagnosis: a comparison of clinical, survey, and American College of 
Rheumatology criteria. Arthritis \& Rheumatism 2005;54(1): $169-76$.

\section{Kerr 2011}

Kerr CE, Shaw JR, Conboy LA, Kelley JM, Jacobson E, Kaptvhuk TJ. Placebo acupuncture as a form of ritual touch healing: a neuro-phenomenological model. Consciousness and Cognition 2011;Article in press:1-8.

\section{Langevin 2011}

Langevin HM, Wayne PM, MacPherson H, Schnyer R, Milley RM, Napadow V, et al.Paradoxes in acupuncture research: strategies for moving forward. Evidence Based Complementary and Alternative Medicine 2011;2011 (180805):1-11.

\section{Langhorst 2010}

Langhorst J, Klose P, Musial F, Irnich D, Hauser W. Efficacy of acupuncture in fibromyalgia syndrome: a systematic review with a meta-analysis of controlled clinical trials. Rheumatology (Oxford) 2010;49(4):778-88.

\section{Lawson 2006}

Lawson K. Emerging pharmacological therapies for fibromyalgia. Current Opinion Investigating Drugs 2006;7 (7):631-6

\section{Lewith 1983}

Lewith GT, Machin D. On the evaluation of the clinical effects of acupuncture. Pain 1983;16:111-27.

\section{Linde 2007}

Linde K, Witt CM, Streng A, Weidenhammer W, Wagenpfeil S, Brinkhaus B, et al.The impact of patient expectations on outcomes in four randomised controlled trials of acupuncture in patients with chronic pain. Pain 2007;128(3):264-71.

Linde 2009

Linde K, Allais G, Brinkhaus B, Manheimer E, Vickers A, White AR. Acupuncture for tension-type headache. Cochrane Database of Systematic Reviews 2009, Issue 1. [DOI: 10.1002/14651858.CD007587]

Lundeberg 2007

Lundeberg T, Lund I. Are reviews based on sham acupuncture procedures in fibromyalgia syndrome (FMS) valid?. Acupuncture in Medicine 2007;25(3):100-6.

\section{MacPerson 2001}

MacPherson H, Thomas K, Walters S, Fitter M. A prospective survey of adverse events and treatment reactions following 34,000 consultations with professional acupuncturists. Acupuncture in Medicine 2001;19(2): 93-102.

\section{MacPherson 2002}

MacPherson H, White A, Cummings M, Jobst K, Rose K, Niemtzow R. Standards for reporting interventions in controlled trials of acupuncture: the STRICTA recommendations. Acupuncture in Medicine 2002;20(1): $22-5$

\section{MacPherson 2004}

MacPherson H, Scullion A, Thomas KJ, Walters S. Patients reports of adverse events associated with acupuncture treatments: a prospective national survey. Quality \& Safety in Health Care 2004;13(5):349-55.

\section{Martin-Sanchez 2009}

Martin-Sanchez E, Torralba E, Diaz-Dominguez E, Barriga A, Martin JLR. Efficacy of acupuncture for the treatment of fibromyalgia: systematic review and meta-analysis of randomised trials. Open Rheumatology Journal 2009;3: 25-9.

\section{Mayhew 2007}

Mayhew E, Ernst E. Acupuncture for fibromyalgia: a systematic review of randomised clinical trials. Rheumatology (Oxford) 2007;46(5):801-4.

\section{Mease 2005}

Mease P. Fibromyalgia syndrome: review of clinical presentation, pathogenesis, outcome measures, and treatment. Journal of Rheumatology 2005;32(Suppl 75): 6-21.

\section{Mease 2009}

Mease P, Arnold LM, Choy EH, Claw DJ, Crofford L, Glass JM, et al.Fibromyalgia syndrome module at OMERACT 9. Journal of Rheumatology 2009;36(10):2318-29.

\section{Mense 2003}

Mense S. The pathogenesis of muscle pain. Current Pain and Headaches Reports 2003;7(6):419-25.

\section{Muller 2007}

Muller W, Schneider EM, Stratz T. The classification of fibromyalgia syndrome. Rheumatology International 2007; 27:1005-10

\section{Ni 1995}

Ni M. The Yellow Emperor's Classic of Medicine. Boston: Shambhala Publications, Inc., 1995.

\section{NIH 1998}

National Institutes of Health. NIH Consensus Conference. Acupuncture. JAMA 1998;280(17):1518-24.

\section{Nüiesch 2012}

Nüesch E, Häuser W, Bernardy K, Barth J, Jüni P. Comparative efficacy of pharmacological and nonpharmacological interventions in fibromyalgia syndrome: network meta-analysis. Annals of the Rheumatic Disease 2012 Jun 27 [Epub ahead of print].

\section{Pioro-Boisset 1996}

Pioro-Boisset M, Esdaile JM, Fitzcharles MA. Alternative medicine use in fibromyalgia syndrome. Arthritis Care Research 1996;9(1):13-7.

\section{Pomeranz 1988}

Le Bars D, Willer JC, de Broucker T, Villanueva L. Neurophysiological mechanisms involved in the painrelieving effects of counter-irritation and related techniques including acupuncture. In: Pomeranz B, Stux G editor (s). Scientific Bases of Acupuncture. Berlin: Springer Verlag, 1988:79-112.

Price 2005

Price DD, Staud R. Neurobiology of fibromyalgia syndrome. Journal of Rheumatology 2005;32(Suppl 75): $22-8$. 


\section{Schneider 2005}

Schneider MJ, Brady DM, Perie SM. Commentary: differential diagnosis of fibromyalgia syndrome: proposal of a model and algorithm for patients presenting with the primary symptom of chronic widespread pain. Journal of Manipulative and Physiological Therapeutics 2006;29: 493-501.

Scott 2006

Scott SW, Deare JC. Acupuncture for migraine: a systematic review. Australian Journal of Acupuncture and Chinese Medicine 2006;1(1):3-14.

\section{Sherman 2002}

Sherman KJ, Hogboom CJ, Cherkin DC, Deyo RA. Description and validation of a non-invasive placebo acupuncture procedure. Journal of Alternative and Complementary Medicine 2002;8:11-9.

\section{Sims 1997}

Sims J. The mechanism of acupuncture analgesia: a review. Complementary Therapies in Medicine 1997;5(2):102-11.

\section{Sutton 2000}

Sutton AJ, Duval SJ, Tweedie RL, Abrams KR, Jones DR. Empirical assessment of effect of publication bias on metaanalyses. BMJ 2000;320(7249):1574-7.

\section{Vickers 2012}

Vickers AJ, Cronin AM, Maschino AC, Lewith G, MacPherson H, Foster NE, et al.Acupuncture for chronic pain. Archives of Internal Medicine 2012;September 10: E1-E10.

Vincent 2001

Vincent C. The safety of acupuncture. BMJ 2001;323 (7311):467-8.

\section{Wallace 2005}

Wallace DJ, Clauw DJ. Fibromyalgia \& Other Central Pain Syndromes. Philadelphia: Lippincott Williams \& Wilkins, 2005.

\section{White 2001}

White A, Hayhoe S, Hart A, Ernst E. Survey of adverse events following acupuncture (SAFA): a prospective study of 32,000 consultations. Acupuncture in Medicine 2001;19 (2):84-92.

\section{WHO 2002}

World Health Organization. Collection of WHO's documents for acupuncture. Beijing, China: The World Federation of Acupuncture-Moxibustion Societies (WFAS), 2002.

\section{WHO 2007}

World Health Organization. WHO International Standard Terminologies on Traditional Medicine in the Western Pacific Region. Manila, Philippines: World Health Organization, 2007. [: ISBN 9789290612487]

\section{Wilke 2009}

Wilke WS. New developments in the diagnosis of fibromyalgia syndrome: say goodbye to tender points?. Cleveland Clinic Journal of Medicine 2009;76(6):345-52.

\section{Wilson 2009}

Wilson HD, Robinson JP, Turk DC. Toward the identification of symptom patterns in people with fibromyalgia. Arthritis \& Rheumatism 2009;61(4):527-34.

\section{Wolfe 1990}

Wolfe F, Smythe HA, Yunas MB, Bennett RM, Bombardier C, Goldenberg DL, et al.The American College of Rheumatology criteria for the classification of fibromyalgia. Report of the Multicenter Criteria Committee. Arthritis and Rheumatism 1990;33(2):160-72.

\section{Wolfe 2003}

Wolfe F, Editorial. Stop using the American College of Rheumatology criteria in the clinic. Journal of Rheumatology 2003;8:1671-2.

\section{Wolfe 2010}

Wolfe F, Clauw DJ, Fitzcharies MA, Goldenberg DL, Katz RS, Mease P, et al.The American College of Rheumatology preliminary diagnostic criteria for fibromyalgia and measurement of symptom severity. Arthritis Care \& Research 2010;62(5):600-10.

\section{Zheng 2010}

Zheng Z, Feng SJQ, da Costa C, Li CC, Lu D, Xue CC. Acupuncture analgesia for temporal summation of experimental pain: a randomised controlled study. European Journal of Pain 2010;14(7):725-31.

* Indicates the major publication for the study 


\title{
CHARACTERISTICS OF STUDIES
}

\section{Characteristics of included studies [ordered by study ID]}

\author{
Assefi 2005
}

Methods

Randomised: computer-generated blocked random allocation sequence with block size of 4 . Researcher not involved in the study conducted randomisation (used academic research centre)

Blinding: participants blinded during treatment. Staff who collected and analysed the data were blinded to treatment group. Care givers were not blinded to group allocation Setting: individual private offices, does not state where these are

Was study aim clear: yes

Informed consent: verbal and written

Ethics approval: institutional review boards at participating institution

WHO clinical trial register: listed and outcomes as per listing

Intention-to-treat used: yes

Follow-up: 3 and 6 months

Participants

Interventions
Total number of participants: 100 participants, mean duration of illness (years): directed acupuncture: 6 years SD 5; sham control groups: acupuncture for unrelated condition 5 years SD 3; sham needling 7 years SD 6; simulated acupuncture 7 years SD 4 2 male and 94 female

Mean age: directed acupuncture; 46 years SD 11; sham control groups: acupuncture for unrelated condition 46 years SD 11; sham needling 49 years SD 14; simulated acupuncture 48 years SD 10

Diagnosis: ACR

Acupuncture-naive participants: yes

Excluded: other pain conditions, contraindicated for acupuncture (bleeding disorders, severe needle phobia), pregnant or breastfeeding, use of narcotics, litigation and previous acupuncture treatments

Recruitment source: the Greater Seattle, Washington State metropolitan area using newspaper, television, university-affiliated hospitals, local fibromyalgia support groups and health care providers

Previous treatments: manual (physical, ergonometric, chiropractic, massage), mental health therapies (psychotherapy, cognitive behavioural therapy), dietary changes or other (nerve blocks, hypnosis or biofeedback)

1) Real: directed acupuncture

Randomised to this group: 25 (analysed 25)

2) Control: acupuncture for unrelated condition, treating for irregular menses or early menses due to Blood Heat

Randomised to this group: 25 (analysed 25)

3) Control: sham needling, using body points not recognised as true acupuncture points Randomised to this group 24 ( 1 did not complete baseline questionnaire) (analysed 24)

4) Control: simulated acupuncture; same acupuncture points as directed acupuncture but with toothpick inside a needle guide tube to mimic needle insertion/withdrawal Randomised to this group 25 (analysed 25)

Minimum number of treatments needed: possible 24 treatments, required to attend $80 \%$ $(19 / 24)$ 
Co-interventions: maintain current use of pharmacological and non-pharmacological therapies through out the study

Acupuncturists: 8 US trained and licensed with median of 10 years experience (range 4 to 18 years)

See STRICTA table for treatment details (Appendix 10)

Outcomes

\section{Primary outcomes:}

1) Pain; visual analogue scale (VAS) $(0=$ no pain, $10=$ worst ever $)$

2) Function: Short-Form 36 health survey, mean of 50 and standard deviation of 10 , with higher scores indicating better functioning

Secondary outcomes:

3) Fatigue: VAS $(0=$ none, $10=$ worst ever $)$

4) Sleep: VAS ( $0=$ worst ever, 10 = best ever $)$

5) Over well-being: VAS ( 0 = worst ever, 10 = best ever $)$

Outcome measures primary and secondary: 1), 2), 3), 4), 5) taken at weeks 1, 4, 8, 12 and 3 and 6 months

6) Blinding: participants rated how certain they were that they had received directed acupuncture or stimulated acupuncture on a 7 -point scale $(1=$ very sure, $7=$ very uncertain) measured at 12 weeks

7) Acupuncturist: participants rated acupuncturist skill level ( $1=$ high, $7=$ low $)$ and adverse events measured at weeks 1, 4, 8 and 12

8) Other co-interventions: medication use measured at week 1 and week 12

Outcome measure results:

"No significant differences were detected between the directed acupuncture and the pooled control group for any of the study outcomes"

For the blinding procedures, " $32 \%$ believed they were receiving acupuncture specifically designed for FM"; no significant difference between the groups $(\mathrm{P}>0.2)$. 4\% believed they were receiving simulated acupuncture; no difference between the groups $(P>0.2)$ Combined groups had no significant difference in the skill of the acupuncturist $(\mathrm{P}>0$. 2). $77 \%$ rated their skill as high, $5 \%$ as medium, $17 \%$ did not know

$84 \%$ in the directed group and $79 \%$ of the pooled sham groups completed the full course of treatment. On average $21 / 24(\mathrm{P}>0.2)$

Total medication use showed no significant difference between groups $(P>0.2)$. Most commonly used medications were ibuprofen, acetaminophen and naproxen

Withdrawals/drop-outs: 4 in at the randomised stage, 10 at the allocated intervention stage, directed group 2, unrelated acupuncture treatment 2, sham acupuncture 2 and simulated acupuncture 4

Complications/adverse events: 89 participants reported adverse events. 37\% reported discomfort at site of needle insertion or simulation of needles, $3 \%$ reported nausea, $0.3 \%$ felt faint. Participants in simulated acupuncture (39\%) had less discomfort than directed acupuncture (61\%), while unrelated acupuncture (70\%) and sham acupuncture (64\%) were similar to directed. Bruising was reported less in the simulated acupuncture group $(10 \%)$, while directed acupuncture $(52 \%)$ it was reported more, the unrelated treatments (74\%) was the worse, with sham acupuncture (68\%) being similar to the last 2 groups. Data extraction methods: data were extracted from the published paper. We selected the 'directed acupuncture' group as the real acupuncture treatment and we combined all the control arms as the sham acupuncture control as per the Cochrane Handbook and confirmed with the Cochrane editors. Mean data were measured from Figure 2 in the 


\begin{tabular}{|c|c|c|}
\hline & \multicolumn{2}{|c|}{$\begin{array}{l}\text { published data and SD was taken from baseline as this was not provided in the figure and } \\
\text { was not published anywhere else. For the comparison of invasive and non-invasive sham } \\
\text { controls we used the 'simulated acupuncture' as it was the same tool (Sherman 2002) as } \\
\text { used in both the Harris } 2008 \text { and Harris } 2009 \text { studies. We could not extract data from } \\
\text { the SF-36 graphs (Figure 3) as both graphs were labelled as SF-36 Physical Component }\end{array}$} \\
\hline Notes & \multicolumn{2}{|c|}{$\begin{array}{l}\text { Other info: } 1 \text { author was contacted by e-mail and confirmed intention-to-treat, however } \\
\text { no further information was given about data or in response to other questions we had } \\
\text { Refunded costs to participants: not reported } \\
\text { Funding: NCCAM } \\
\text { Language: English } \\
\text { Publication: full paper }\end{array}$} \\
\hline \multicolumn{3}{|l|}{ Risk of bias } \\
\hline Bias & Authors' judgement & Support for judgement \\
\hline $\begin{array}{l}\text { Random sequence generation (selection } \\
\text { bias) }\end{array}$ & Low risk & Computer-generated random number \\
\hline Allocation concealment (selection bias) & Low risk & $\begin{array}{l}\text { Blocked random allocation sequence with } \\
\text { block size of } 4 \text { with an independent re- } \\
\text { searcher advising the acupuncture clinic of } \\
\text { treatment assignment }\end{array}$ \\
\hline $\begin{array}{l}\text { Incomplete outcome data (attrition bias) } \\
\text { All outcomes }\end{array}$ & Low risk & $\begin{array}{l}\text { Data from } 7 \text { participants were not included } \\
\text { in the analysis. However, the missing out- } \\
\text { come data were balanced across the groups } \\
\text { and less likely to have impacted on the out- } \\
\text { come }\end{array}$ \\
\hline Selective reporting (reporting bias) & Low risk & $\begin{array}{l}\text { All outcomes reported, as per WHO clini- } \\
\text { cal trials register }\end{array}$ \\
\hline $\begin{array}{l}\text { Blinding of participants and personnel } \\
\text { (performance bias) } \\
\text { All outcomes }\end{array}$ & Low risk & $\begin{array}{l}\text { Restricted conversation during treatment, } \\
\text { participants blindfolded and } \\
\text { used acupuncture-naive participants. Care } \\
\text { giver not blinded to group allocation. Par- } \\
\text { ticipants tested for blinding could not de- } \\
\text { tect which group they belonged to at the } \\
\text { end of treatment }\end{array}$ \\
\hline $\begin{array}{l}\text { Blinding of outcome assessment (detection } \\
\text { bias) } \\
\text { All outcomes }\end{array}$ & Low risk & $\begin{array}{l}\text { Data collection staff and data analysts were } \\
\text { blinded to treatment group }\end{array}$ \\
\hline
\end{tabular}


Methods

Participants
Randomised: electronic number generator, closed envelopes, numbered 1 to 70 , prepared before study and opened in numerical order after recruitment

Blinding: participants and outcomes assessors were blinded. Care giver was NOT blinded to group allocation

Setting: University Hospital, Geneva, Switzerland

Was study aim clear: yes

Informed consent: verbal and written

Ethics approval: Department of Medicine ethics committee

WHO clinical trial register: not listed as had not been established when study undertaken Follow-up: none

Intention-to-treat used: not stated, but in the results section it states that the 15 participants that withdraw were not re-evaluated. Martin 2006 in their journal article stated that analysis used intention-to-treat, yet the review by Berman 1999 using intention-to-treat, found $42 \%$ had no benefit, $39 \%$ had satisfactory benefit, while $19 \%$ had an unexpectedly large benefit. In a recent review this detail was omitted by (Mayhew 2007). Assefi 2005 and Harris 2005 also did not mention this point in their discussion about other studies

Total number of participants: 70 participants, mean duration of disease (years); real acupuncture $=14.4$ years $(3.7)(6.9$ to 22.0$)$, control $=6.9$ years $(1.3)(4.3$ to 9.6)

16 male and 54 female (excess of men in the control group $\mathrm{P}=0.015$ )

Mean age (years): real acupuncture $=46.8$, control $=49$

Diagnosis: ACR

Acupuncture-naive participants: yes

Excluded: severe concomitant disease, use of morphine-like drugs or anticoagulants, peripheral neuropathy, bleeding disorders, language difficulties and past use of acupuncture Recruitment source: referred, but does not state where from

Previous treatments: not reported
1) Real: electro-acupuncture (visible muscle contraction)

Randomised to this group: 36 (analysed 28)

2) Control: sham electroacupuncture, similar number of needles except off the acupuncture point by $20 \mathrm{~mm}$ and current used on electro-stimulator was weaker than the real group. No increase in electrical volume was applied once the threshold of perception had been reached

Randomised to this group: 34 (analysed 27)

Co-interventions: individual treatments continued, physiotherapy, anti-inflammatory agents, tricyclic antidepressants and analgesics

See STRICTA table for treatment details (Appendix 10).

1) Pain; visual analogue scale (1 to $100 \mathrm{~mm}$ )

Secondary outcomes:

2) Pain threshold, measured by pressure gauge over the 18 tender points as defined by ACR, before and after treatment

3) Analgesic use, tablets. Initial measurements taken in the week before the evaluation took place

4) Regional pain score, body drawing in which 21 regions are indicated. Patient assesses their pain in each region on a scale of 1 to 5 , with 5 being the worst

5) Sleep quality scale (1 to 10 ), with 10 being the best 
Deluze 1992 (Continued)

6) Morning stiffness, measured in minutes

7) Patient general state ( 1 to 10$)$, measured by patient, with 10 being the best

8) Evaluating physician impression (1 to 10$)$, measured by physician as to the patient's general state, with 10 being the best

Outcome measures taken before and after treatments completed

Outcome measure results:

Overall approximately 50\% improved significantly, 25\% had no change with the balance showing "unexpectedly large improvement, with almost complete disappearance of symptoms"; 1 in the control group was observed to have a similar result

Real group improved significantly in 5 out of the 8 areas except morning stiffness

Pain threshold improved by $70 \%$ in the real group as opposed to $4 \%$ in the control group Withdrawals/drop-outs: real acupuncture $=8$, control group $=7$

Complications/adverse events: real electroacupuncture $6(2=$ increase in symptoms, $3=$ unpleasantness of needle sensation, $1=$ ankle oedema). Sham electroacupuncture 5 ( $4=$ increase in symptoms, 1 = unpleasantness of needle sensation)

Data extraction method: data were extracted from the published paper using table 2 and ZZ converted the SE data to SD. We selected the 'VAS pain scale' rather than the 'regional pain score' as it was the most used measurement tool for pain

Other info: e-mail contact was made with lead author who stated they were too busy to answer questions

Refunded costs to participants: not reported

Funding: not stated

Language: English

Publication: full paper

\section{Risk of bias}

\section{Bias}

bias)

\section{Support for judgement}

Electronic number generator

Closed envelopes, numbered 1 to 70, prepared before study and opened in numerical order after recruitment

Incomplete outcome data (attrition bias) High risk All outcomes
11 participants dropped out from the study, and their data were not included in the whole analysis. Number of participants dropped out from the study and reasons for drop-out were comparable in both groups $(5 / 27 ; 6 / 28)$. As nearly $80 \%$ of those dropped out were due to increased symptoms, attrition bias is possible although this was comparable in both treatment groups

WHO clinical trials register was not established at time of publication 


\section{Deluze 1992 (Continued)}

Blinding of participants and personnel Low risk (performance bias)

All outcomes

Blinding of outcome assessment (detection Low risk bias)

All outcomes
Used acupuncture-naive participants, care giver not blinded

Independent evaluator, unaware of group allocations

Guo 2005

Methods

Participants
Randomised: semi-randomised according to the order of admission

Blinding: patient/care giver were not blinded. Whether assessors were blinded is not reported.

Setting: in and outpatients of an affiliated hospital, Helongjiang, China

Was study aim clear: no

Informed consent: not reported

Ethics approval: not reported

WHO clinical trial register: not listed

Follow-up: 6 months

Intention-to-treat used: not reported

Total number of participants: 38 patients, mean duration of illness: acupuncture: 11 (2. 3) months; control: 10 (3.6) months

7 male and 31 female; acupuncture group: M:F 3:16; control: M:F 4:15

Mean age: real acupuncture group $=50(2.9) \mathrm{yrs}($ not sure if this is SD); control $=49$ (3.

4)

Diagnosis: ACR

Acupuncture-naive participants: not reported

Excluded: not reported (did not mention if there were exclusion criteria)

Recruitment source: not reported.

Previous treatments: not reported

Interventions

1) Real: acupuncture group

Randomised to this group: 19 (analysed 19)

2) Control: Western medication group (amitriptyline, tricyclic antidepressant, start from

$10 \mathrm{mg}$, increased by $10 \mathrm{mg}$ every 10 days until $30 \mathrm{mg}$, dividing dose into 2 and taking them at 2 different times of the day, 30 days 1 course)

Randomised to this group: 19 (analysed 19)

Co-interventions: not reported

See STRICTA table for treatment details (Appendix 10)

Outcomes

Primary outcome:

1) Pain; visual analogue scale (VAS), did not specify either 1 to 10 or 0 to 100 range Secondary outcome:

2) Number of tender points, did not specify details

Outcome measure results:

Symptoms and signs-free, no tender points; acupuncture 9; control: 3

Significantly improved: VAS and tender points both reduced by or over $50 \%$; most 


\begin{tabular}{|c|c|c|}
\hline & \multicolumn{2}{|c|}{$\begin{array}{l}\text { symptoms and signs resolved; } 5: 2 \\
\text { Improved: VAS and tender points both reduced by } 25 \% \text { to } 50 \% \text {; some improvement in } \\
\text { S/S; } 4: 8 \\
\text { No effect: VAS and tender points both reduced }<25 \% \text {, no changes in S/S; } 1: 6 \\
\text { Withdrawals/drop-outs: no reported but based on data there were none } \\
\text { Complications/adverse events: not reported } \\
\text { Data extraction method: data were extracted from published paper table } 2\end{array}$} \\
\hline Notes & \multicolumn{2}{|c|}{$\begin{array}{l}\text { Other info: we could not contact the lead author to clarify missing information } \\
\text { Refunded costs to participants: not reported } \\
\text { Funding: not reported } \\
\text { Language: Chinese } \\
\text { Publication: full paper }\end{array}$} \\
\hline \multicolumn{3}{|l|}{ Risk of bias } \\
\hline Bias & Authors' judgement & Support for judgement \\
\hline $\begin{array}{l}\text { Random sequence generation (selection } \\
\text { bias) }\end{array}$ & High risk & RCT, but no other information \\
\hline Allocation concealment (selection bias) & High risk & $\begin{array}{l}\text { Semi-randomised according to the order of } \\
\text { admission }\end{array}$ \\
\hline $\begin{array}{l}\text { Incomplete outcome data (attrition bias) } \\
\text { All outcomes }\end{array}$ & Low risk & $\begin{array}{l}\text { No drop-out reported. According to the } \\
\text { data provided, all participants were in- } \\
\text { cluded in the analysis }\end{array}$ \\
\hline Selective reporting (reporting bias) & Unclear risk & Not listed on WHO clinical trials register \\
\hline $\begin{array}{l}\text { Blinding of participants and personnel } \\
\text { (performance bias) } \\
\text { All outcomes }\end{array}$ & Unclear risk & Not reported \\
\hline $\begin{array}{l}\text { Blinding of outcome assessment (detection } \\
\text { bias) } \\
\text { All outcomes }\end{array}$ & Unclear risk & Not reported \\
\hline
\end{tabular}


Methods

Interventions
Randomised: computer-generated random numbers in a 4-block design. Concealed in an opaque envelope and given to the acupuncturist 1 day before treatment.

Blinding: participants blindfolded with a non-blinded research assistant present during the treatments to monitor and ensure treatment integrity. Outcome assessors were blinded to treatment allocation. Care givers knew the allocation groups and the hypothesis

Setting: Georgetown University, Washington DC

Was study aim clear: yes

Informed consent: verbal and written

Ethics approval: Institutional Review Board

WHO clinical trial register: listed on site and outcomes as per listing

Follow-up: none

Intention-to-treat: yes

Total number of participants: 114 participants, mean duration of illness (years): T/S $=5$. 50 years $(3.71), \mathrm{T} / \mathrm{O}=5.26$ years $(4.83), \mathrm{N} / \mathrm{S}=5.17$ years $(4.24), \mathrm{N} / \mathrm{O}=5.77$ years $(4$. 10)

8 male and 106 female

Mean age: T/S 46.0 (10.1), T/O 44.5 (10.9), N/S 51.3 (10.0), N/O 48.1 (10.9)

Diagnosis: ACR

Acupuncture-naive participants: yes

Excluded: previous acupuncture treatments including sufficient knowledge that would prevent blinding, bleeding diathesis, autoimmune or inflammatory disease, daily narcotic analgesic use or a history of substance abuse, contraindication to use of acetaminophen or ibuprofen, in other clinical studies, pregnancy or lactation, receiving disability payment or litigation related to fibromyalgia

Recruitment source: the Washington DC metropolitan area using newspaper, periodicals and screened by telephone

Previous treatments: not reported

1) Real: traditional acupuncture (T/S) with stimulation

Randomised to this group: 29

2) Control: traditional acupuncture $(\mathrm{T} / \mathrm{O})$ without stimulation

Randomised to this group: 30

3) Control: non-traditional acupuncture $(\mathrm{N} / \mathrm{S})$ with stimulation, needles at same depth and stimulation as T/S group. Needles were placed in sites not believed to effective in Traditional Chinese Medicine based acupuncture

Randomised to this group: 28

4) Control: non-traditional acupuncture (N/O) without stimulation, in non-traditional sites

Randomised to this group: 27

(Each group received treatment once per week for 3 weeks, then twice per week for 3 weeks, then 3 times per week for 3 weeks (total 18 treatments). Between each treatment, there was a 2-week washout period)

Minimum number of treatments needed: not reported

Co-interventions: participants were allowed to continue normal treatments including antidepressants. They were not allowed to make any changes during the trial and not to seek acupuncture outside of the trial

See STRICTA table for treatment details (Appendix 10) 
Harris 2005 (Continued)

\begin{tabular}{|c|c|}
\hline Outcomes & $\begin{array}{l}\text { Primary outcomes: } \\
\text { 1) Pain: numeric rating scale, } 101 \text {-point, range from } 0 \text { to } 100 \text { points in } 5 \text {-point incre- } \\
\text { ments, } 0=\text { no pain to } 100=\text { worst pain imaginable (assessed before and week } 3,4 \text { to } 5 \text {, } \\
8,9 \text { to } 10,13,14 \text { to } 15 \text { ) } \\
\text { 2) Function: Short-Form } 36 \text {, score ranges from } 0 \text { to } 100 \text { with higher scores indicating } \\
\text { better function (assessed before and week } 4 \text { to } 5,9 \text { to } 10,14 \text { to } 15 \text { ) } \\
\text { Secondary outcomes: } \\
\text { 3) Fatigue: Multi-Dimensional Fatigue Inventory questionnaire, scores range from } 4 \text { to } \\
20 \text { with larger scores indicating more fatigue (assessed before and week } 4 \text { to } 5,9 \text { to } 10 \text {, } \\
14 \text { to } 15 \text { ) } \\
\text { 4) Blinding: participants were asked in week } 4 \text { whether they could determine which } \\
\text { treatment arm they were in (A = acupuncture, B = placebo and C = could not tell) } \\
\text { Outcome measure results: } \\
\text { "Clinically significant improvements in pain were observed in } 25 \% \text { to } 35 \% \text { of subjects" } \\
\text { "Blinding assessment indicated that participants remained blinded to treatment at week } \\
4 \text { (P = 0.259)" } \\
\text { Withdrawals/drop-outs: } 38 \text { (T/S }=7 \text {, T/O }=11, \mathrm{~N} / \mathrm{S}=8 \text {, N/O = 12) } \\
\text { Complications/adverse events: not reported } \\
\text { Data extraction method: data were extracted from the published paper using Table } 2 \text {. } \\
\text { We selected T/S and N/O data to represent real and sham acupuncture. For comparing } \\
\text { different acupuncture styles we choose T/S versus T/O }\end{array}$ \\
\hline
\end{tabular}

Notes

Other info: contact was made with lead author who confirmed details of drop-outs and location of trial

Refunded costs to participants: not reported

Funding: NCCAM

Language: English

Publication: full paper

Risk of bias

Bias

Random sequence generation (selection Low risk bias)

Allocation concealment (selection bias) Low risk

Incomplete outcome data (attrition bias) Low risk All outcomes

\section{Support for judgement}

Computer-generated

Random numbers in a 4-block design. Concealed in an opaque envelope and given to the acupuncturist 1 day before treatment.

All participants including those who dropped out from the study were included in the data analysis. The number of and reasons for drop-out were comparable among the groups 
Harris 2005 (Continued)

\begin{tabular}{l|l|l}
$\begin{array}{l}\text { Selective reporting (reporting bias) } \\
\text { Blinding of participants and personnel } \\
\text { (performance bias) } \\
\text { All outcomes }\end{array}$ & Low risk & $\begin{array}{l}\text { All outcomes reported, as per WHO clini- } \\
\text { cal trials register }\end{array}$ \\
\hline & $\begin{array}{l}\text { Used } \\
\text { acupuncture-naive participants, other par- } \\
\text { ticipants not present at clinic during treat- } \\
\text { ment, blindfolded during treatment. Care } \\
\text { giver not blinded. Non-blinded research as- } \\
\text { sistant present during all treatments to en- } \\
\text { sure treatment integrity. Blinding of allo- } \\
\text { cation groups was tested at week 3 with no } \\
\text { significant differences noted }\end{array}$ \\
\hline
\end{tabular}

Blinding of outcome assessment (detection Low risk bias)

All evaluators blinded to treatment allocation

All outcomes

\section{Harris 2008}

Methods

Participants
Randomised: random number generator used (blocks of 4 with 2 acupuncture and 2 sham)

Blinding: participants blindfolded during treatments. All assessors were blinded to treatment assignments

Setting: University of Michigan, USA

Was study aim clear: yes

Informed consent: written and informed

Follow-up: none, only for term of treatment

Ethics approval: University of Michigan Institutional Review Board

WHO clinical trial register: not listed and confirmed by author

Intention-to-treat: yes, all subjects completed trial

Total number of participants: 10 participants, duration of fibromyalgia for $>1$ year 0 male (acupuncture and control) and 10 female

Mean age: both acupuncture and control combined mean 48 SD 15 years

Diagnosis: ACR 1990 criteria

Acupuncture-naive participants: yes

Excluded: as per Harris 2005 study

Recruitment source: fibromyalgia subject registry at the University of Michigan Chronic

Pain and Fatigue Center

Previous treatments:

Interventions
1) Real: acupuncture

Randomised to this group: 6

2) Control: non skin-penetrating acupuncture (Sherman 2002)

Randomised to this group: 4

Minimum number of treatments needed: 9 out of 9

Co-intervention: none

See STRICTA table for treatment details (Appendix 10) 
Harris 2008 (Continued)

\begin{tabular}{|c|c|}
\hline Outcomes & $\begin{array}{l}\text { Primary outcomes: } \\
\text { 1) Pain: VAS subset of Short-Form McGill Pain Questionnaire (SF-MPQ) } \\
\text { Assessments: at baseline and end of treatment } \\
\text { Outcome measure results: clinical pain improved from pre- to post-treatment according } \\
\text { to SF-MPQ rating of the sensory dimensions of pain (mean difference in clinical pain } \\
\text { rating } 3.50 \text { (SD 4.70); P = } 0.043 \text { ) } \\
\text { SF-MPQ sensory score baseline mean (SD) = } 12.3(4.35) \\
\text { SF-MPQ sensory score end of treatment mean (SD) = } 8.80(5.61) \\
\text { Withdrawals/drop-outs: no drop-outs } \\
\text { Complications/adverse events: no adverse events } \\
\text { Author stated "actually the primary outcome for this study was neuroimaging changes for } \\
\text { TA and SA. Clinical pain was never a primary outcome, it was only used as a covariate" } \\
\text { Data extraction method: data for pain was provided by the author directly }\end{array}$ \\
\hline Notes & $\begin{array}{l}\text { Other info: part of an ongoing study; results for this study have not been published } \\
\text { anywhere else. Missing details from the study were confirmed by the lead author via } \\
\text { e-mail. They included details of randomisation, blinding, whether acupuncture-naive, } \\
\text { score baseline/end of treatment/drop-outs and data. The lead author confirmed that this } \\
\text { is not a subset of the Harris } 2005 \text { study. } \\
\text { Funding: US Department of Army grant and NIH/National Centre for Complementary } \\
\text { and Alternative Medicine } \\
\text { Refunded costs to participants: not reported } \\
\text { Language: English } \\
\text { Publication: full paper }\end{array}$ \\
\hline
\end{tabular}

Risk of bias

\begin{tabular}{|c|c|c|}
\hline Bias & Authors' judgement & Support for judgement \\
\hline $\begin{array}{l}\text { Random sequence generation (selection } \\
\text { bias) }\end{array}$ & Low risk & Computer-generated \\
\hline Allocation concealment (selection bias) & Low risk & $\begin{array}{l}\text { Random number generator used (blocks of } \\
4 \text {, with } 2 \text { acupuncture and } 2 \text { sham) }\end{array}$ \\
\hline $\begin{array}{l}\text { Incomplete outcome data (attrition bias) } \\
\text { All outcomes }\end{array}$ & Low risk & No missing data \\
\hline Selective reporting (reporting bias) & Unclear risk & Not listed on WHO clinical trials register \\
\hline $\begin{array}{l}\text { Blinding of participants and personnel } \\
\text { (performance bias) } \\
\text { All outcomes }\end{array}$ & Low risk & $\begin{array}{l}\text { Used acupuncture-naive participants, par- } \\
\text { ticipants blindfolded, care giver was not } \\
\text { blinded }\end{array}$ \\
\hline $\begin{array}{l}\text { Blinding of outcome assessment (detection } \\
\text { bias) } \\
\text { All outcomes }\end{array}$ & Low risk & All assessments were blinded \\
\hline
\end{tabular}

Acupuncture for treating fibromyalgia (Review)

Copyright @ 2013 The Cochrane Collaboration. Published by John Wiley \& Sons, Ltd. 
Methods

Participants
Randomised: random number generator used (blocks of 4 with 2 acupuncture and 2 sham)

Blinding: participants blindfolded during treatments. All assessors were blinded to treatment assignments

Setting: University of Michigan, USA

Was study aim clear: yes

Informed consent: written and informed

Follow-up: none, only for term of treatment

Ethics approval: University of Michigan Institutional Review Board

WHO clinical trial register: not listed and confirmed by author

Intention-to-treat: yes, all subjects completed trial

Total number of participants: 20 participants, duration of fibromyalgia > 1 year 0 male (acupuncture and control) and 20 female

Mean age: both acupuncture and control combined mean 44.3 SD 13.6 years

Diagnosis: ACR 1990 criteria

Acupuncture-naive participants: yes

Excluded: as per Harris 2005 study

Recruitment source: fibromyalgia subject registry at University of Michigan

Previous treatments: not reported

Interventions

1) Real: acupuncture (TA)

Randomised to this group: 10

2) Control: non skin-penetrating acupuncture (SA) (Sherman 2002)

Randomised to this group: 10

Minimum number of treatments needed: 9 out of 9

Co-intervention: medication (agreed not to change)

See STRICTA table for treatment details (Appendix 10)

Outcomes

Primary outcomes:

1) Pain: McGill Pain Questionnaire (SF-MPQ)

Assessments: st baseline and end of treatment

Outcome measure results: "significant reductions in pain were observed for the entire cohort for the total score SF-MPQ"

Total: mean difference (SD) treatment - baseline: -3.45 (7.39); $\mathrm{P}=0.05$

SF-MPQ Sensory Score: mean (SD): -2.65 (5.98) P = 0.06

SF-MPQ Affective Score: mean (SD): $-0.80(2.25) \mathrm{P}=0.13$

Both TA and SA resulted in clinically meaningful reductions in pain (SF-MPQ total score mean difference (SD): TA -4.00 (6.72); SA -2.90 (8.33)

2) Assessment of masking:

Participants had to guess which group they belonged to after the first PET scan. Overall the 2 distributions were not statistically different: $\mathrm{Chi}^{2}=0.88, \mathrm{P}=0.65$

Withdrawals/drop-outs: no drop-outs

Complications/adverse events: no adverse events

Author stated "actually the primary outcome for this study was neuroimaging changes for TA and SA. Clinical pain was never a primary outcome, it was only used as a covariate" Data extraction method: data were provided by the author. The author noted that "actually in this manuscript (Harris 2009) we did not analyse real versus sham acupuncture groups. We combined both groups together in this analysis" 
Harris 2009 (Continued)

Other info: part of an ongoing study. Details of study were confirmed by the author via
e-mail: drop-outs/adverse events and data. The author confirmed that this is not a subset
study of Harris 2005.
Funding: US Department of Army grant and NIH/National Centre for Complementary
and Alternative Medicine
Refunded costs to participants: not reported
Language: English
Publication: full paper

\section{Risk of bias}

\begin{tabular}{|c|c|c|}
\hline Bias & Authors' judgement & Support for judgement \\
\hline $\begin{array}{l}\text { Random sequence generation (selection } \\
\text { bias) }\end{array}$ & Low risk & Computer-generated \\
\hline Allocation concealment (selection bias) & Low risk & $\begin{array}{l}\text { Random number generator used (blocks of } \\
4 \text {, with } 2 \text { acupuncture and } 2 \text { sham) }\end{array}$ \\
\hline $\begin{array}{l}\text { Incomplete outcome data (attrition bias) } \\
\text { All outcomes }\end{array}$ & Low risk & No missing data \\
\hline Selective reporting (reporting bias) & Unclear risk & Not listed on WHO clinical trials register \\
\hline $\begin{array}{l}\text { Blinding of participants and personnel } \\
\text { (performance bias) } \\
\text { All outcomes }\end{array}$ & Low risk & $\begin{array}{l}\text { Used acupuncture-naive participants, par- } \\
\text { ticipants blindfolded, care giver was not } \\
\text { blinded. Participants had to guess which } \\
\text { group they belonged to after first PET scan. } \\
\text { Overall the } 2 \text { distributions were not statis- } \\
\text { tically different }\end{array}$ \\
\hline $\begin{array}{l}\text { Blinding of outcome assessment (detection } \\
\text { bias) } \\
\text { All outcomes }\end{array}$ & Low risk & All assessments were blinded \\
\hline
\end{tabular}


Diagnosis: ACR 1990 criteria

Acupuncture-naive participants: yes

Excluded: previous acupuncture, bleeding disorders, autoimmune or inflammatory diseases, participation in other trials, pregnancy or lactation, receiving disability payments or involved in litigation related to fibromyalgia

Recruitment source: fibromyalgia specialists at hospitals

Previous treatments: maintain current medication use

1) Real: acupuncture (TA)

Group B, electro- and trigger point acupuncture

Randomised to this group: 7

2) Control: Group A received 5 acupuncture sessions after 5 weeks of weekly or twice weekly clinical examinations only

Randomised to this group: 6

Minimum number of treatments needed: not reported

Co-intervention: medications using amitriptyline, SSRIs and SNRIs (agreed not to change)

See STRICTA table for treatment details (Appendix 10)

1) Pain: VAS $19 \mathrm{~cm}$ scale with higher score indicating negative impact

2) Function: Fibromyalgia Impact questionnaire FIQ), 20 items covering physical functioning, work status, depression, anxiety, sleep (rest/morning tiredness), pain, stiffness, fatigue and well-being. Each scored 0 to 10 . The higher the combined score the worse the condition is affecting the participant. Full details on scoring can be found in Burckhardt 1991.

Assessments: before start, week 5 and at end of treatments, week 10

Outcome measure results: VAS, Group A remained unchanged until acupuncture treatment started, while Group B decreased by week 5 . No differences between groups at baseline ( $\mathrm{P}=0.566)$, while at week 5 , significant differences in VAS between groups ( $\mathrm{P}$ $=0.022)$ and at week 10 no difference $(\mathrm{P}=0.252)$

FIQ, Group A remained unchanged until acupuncture treatment started, while Group $\mathrm{B}$ decreased by week 5 . No differences between groups at baseline $(\mathrm{P}=0.616)$, while at week 5 significant differences in FIQ between groups $(P=0.026)$ and at week 10 no 


\begin{tabular}{|c|c|c|}
\hline & \multicolumn{2}{|c|}{$\begin{array}{l}\text { difference }(\mathrm{P}=0.86) \\
\text { Withdrawals/drop-outs: Group A 2, Group B 1, both lost due to lack of response to } \\
\text { treatment } \\
\text { Complications/adverse events: none reported } \\
\text { Data extraction method: data were provided by the lead author directly }\end{array}$} \\
\hline Notes & \multicolumn{2}{|c|}{$\begin{array}{l}\text { Other info: author was contacted by e-mail and provided data } \\
\text { Funding: not reported } \\
\text { Refunded costs to participants: not reported } \\
\text { Language: English } \\
\text { Publication: full paper } \\
\text { Results: see the comparisons }\end{array}$} \\
\hline \multicolumn{3}{|l|}{ Risk of bias } \\
\hline Bias & Authors' judgement & Support for judgement \\
\hline $\begin{array}{l}\text { Random sequence generation (selection } \\
\text { bias) }\end{array}$ & Low risk & Computer-generated \\
\hline Allocation concealment (selection bias) & Low risk & $\begin{array}{l}\text { Permutated block randomisation to either } \\
\text { group }\end{array}$ \\
\hline $\begin{array}{l}\text { Incomplete outcome data (attrition bias) } \\
\text { All outcomes }\end{array}$ & High risk & $\begin{array}{l}\text { Intention-to-treat not used, } 3 \text { drop-outs (1 } \\
\text { acupuncture, } 2 \text { no treatment groups) due } \\
\text { to lack of response to treatment, however } \\
\text { drop-out rates were not significantly differ- } \\
\text { ent between groups }\end{array}$ \\
\hline Selective reporting (reporting bias) & Unclear risk & Not listed on WHO clinical trials register \\
\hline $\begin{array}{l}\text { Blinding of participants and personnel } \\
\text { (performance bias) } \\
\text { All outcomes }\end{array}$ & High risk & $\begin{array}{l}\text { Used acupuncture-naive participants, how- } \\
\text { ever participants at start of treatment would } \\
\text { have known which group they belonged } \\
\text { to due to study design; care giver was not } \\
\text { blinded }\end{array}$ \\
\hline $\begin{array}{l}\text { Blinding of outcome assessment (detection } \\
\text { bias) } \\
\text { All outcomes }\end{array}$ & Low risk & $\begin{array}{l}\text { All assessments performed by independent } \\
\text { investigator not aware of treatment se- } \\
\text { quence or treatment received }\end{array}$ \\
\hline
\end{tabular}



Each contained a $3 \times 5$ index card, which was printed with the group assignment. Every 4 envelopes contained 2 control and 2 experimental assignments. This was done in blocks of 2 to prevent imbalances in treatment allocation. Envelopes were opened in order Blinding: participants seated in an arrangement that blocked their view to treatment. Outcome assessors blinded to group allocation. Care givers and participants maintained neutral conversion

Setting: Mayo Fibromyalgia Treatment Program, Rochester, Minnesota, USA

Was study aim clear: yes

Informed consent: verbal and written

Ethics approval: Mayo Foundation Institutional Review Board

WHO clinical trial register: not listed and confirmed by author who stated they did not know about it

Follow-up: 1 and 7 months

Intention-to-treat: yes

Total number of participants: 50 patients, mean duration of illness (years): not reported 1 male (control group) and 49 female (real and control)

Mean age: acupuncture 47.9 SD 11.2 years, control 51.7 SD 14.1 years

Diagnosis: ACR

Acupuncture-naive participants: yes

Excluded: prior acupuncture experience, bleeding diathesis, had to be able to understand consent and to be able to fill out the questionnaires

Recruitment source: referrals to programme from physician after conservative management Previous treatments: patients had received conservative management, but this was not described; "many had already used most of the basic treatments for fibromyalgia"

Interventions

1) Real: electroacupuncture, used a special table arrangement that did not allow the patient to see what was happening;

Randomised to this group: 25

2) Control: sham electroacupuncture, setting was same as real group, except needle was attached to plaster and did not break the skin;

Randomised to this group: 25

Minimum number of treatments needed: all patients completed at least 5 treatments Co-interventions: 1.5 days of education, counselling and group discussion about symptom management (done before enrolment into study, 4-week wash-out period before start of treatments). No other co-interventions were reported

See STRICTA table for treatment details (Appendix 10)

1) Pain: Multidimensional Pain Inventory (MPI), 61-item questionnaire developed for chronic pain. Composed of 13 scales that measure different pain-related aspects. 4 of the questions that related to support from spouse or significant other were excluded, as it was not part of the standard treatment programme

2) Function: Fibromyalgia Impact Questionnaire (FIQ), 20 items covering physical functioning, work status, depression, anxiety, sleep (rest/morning tiredness), pain, stiffness, fatigue and well-being. Each scored 0 to 10 . The higher the combined score the worse the condition is affecting the patient. Full details on scoring can be found in the article by Burckhardt 1991. 


\section{Secondary outcomes:}

3) Participants were asked their opinion regarding group assignment

Assessments: before start, immediately at end of treatment sessions and at 1 and 7 months Outcome measure results:

FIQ showed significant improvement in the acupuncture group over control acupuncture during study period $(P=0.01)$, with the greatest difference at the 1 month $(P=0.007)$. Subscale analysis showed significant group effect for fatigue $(\mathrm{P}=0.001)$ and anxiety $(\mathrm{P}$ $=0.003)$ at 1 month. Other sub-scales showed trends towards improvement but were not statistically significant

MPI group effect showed significant improvement in pain at 1 month $(P=0.03)$ but effect was lost at the 7 -month measure $(\mathrm{P}=0.05)$

Blinding of participants did not exceed chance

Withdrawals/drop-outs: 1 lost to follow-up

Complications/adverse events: many participants in both groups experienced feeling tired and/or relaxed after treatment. Mild bruising and soreness was common in acupuncture group. 2 patients experienced mild vasovagal symptoms (1 from each group). 1 patient experienced a pulmonary embolism (believed to be unrelated to the study).

Data extraction method: data were extracted from published paper table 3 and we used data at the one month after treatment point as per our protocol

Other info: author was contacted by e-mail and confirmed allocation concealment, WHO listing and point locations

Funding: Mayo Foundation and Mayo Anaesthesia Clinical Research Unit

Refunded costs to participants: financial compensation provided for parking

Language: English

Publication: full paper

\section{Risk of bias}

\begin{tabular}{|c|c|c|}
\hline Bias & Authors' judgement & Support for judgement \\
\hline $\begin{array}{l}\text { Random sequence generation (selection } \\
\text { bias) }\end{array}$ & Unclear risk & Random sequence not reported \\
\hline Allocation concealment (selection bias) & Low risk & $\begin{array}{l}\text { Immediately } \\
\text { before first treatment. Opaque envelopes, } \\
\text { labelled sequentially. Each contained a } 3 \mathrm{x} \\
5 \text { index card, which was printed with the } \\
\text { group assignment. Every } 4 \text { envelopes con- } \\
\text { tained } 2 \text { control and } 2 \text { experimental assign- } \\
\text { ments. This was done in blocks of } 4 \text { to } \\
\text { prevent imbalances in treatment allocation. } \\
\text { Envelopes were opened in order }\end{array}$ \\
\hline
\end{tabular}

Incomplete outcome data (attrition bias) Low risk All outcomes
All participants completed outcome measures at the end of treatment; 1 from the control group did not have data for 1 and 7 month follow-ups and was excluded from follow-up analysis. Given this was only 1 
Martin 2006 (Continued)

participant, this exclusion would have minimal impact on the outcome

Selective reporting (reporting bias) Unclear risk

Blinding of participants and personnel Low risk (performance bias)

All outcomes
Not listed on the WHO clinical trials register; author stated did not know about it

Used acupuncture-naive participants, blinded to actual treatment by seated arrangement of protocol, restricted conversation during treatment and use of blinded study co-ordinator for questions. Care giver was not blinded. Group allocation was tested and ability of patients to determine treatment received did not exceed chance

Blinding of outcome assessment (detection Low risk bias)

All outcomes
All evaluations were obtained from participants by study co-ordinator who was blinded to group allocations

\section{Targino 2008}

Methods

Participants
Randomised: using "a computer-generated random sequence of numbers provided by the Hospitals Informatics Departments. The randomization was conducted by one physician who was not involved with the inclusion or exclusion process."

Blinding: assessor blind - "participants rated their pain intensity using a VAS. Blinded evaluation of PPT18 and TePsN was carried out by a single physician (HHSK), while blind evaluation of quality of life was conducted by one psychologist (LPMS). Even though the participants knew which group they were in (either the acupuncture treatment group or the standard care group), they were instructed not to communicate this information to the outcome assessors."

Setting: not reported, but assume it was the Clinics Hospital

Was study aim clear: yes

Informed consent: written and informed consent

Follow-up: 3 months (after randomisation and at the end of the treatment), 6 months, 12 months and 24 months

Ethics approval: the ethics review committee of the Clinics Hospital

WHO clinical trial register: listed and outcomes as per listing

Intention-to-treat: yes, up to 12 months follow-up, but not at 24 months follow-up

Total number of participants: 58 patients, mean duration of pain (months): acupuncture group 118.8 (117.3), control 93.0 (75.25). Did not describe what they meant by pain duration, whether it was fibromyalgia diagnosis or pain. Assume it refers to fibromyalgia Gender: 0 male (acupuncture and control) and 58 female

Mean age: acupuncture 52.09 SD 10.97 years, control 51.17 SD 11.20 years

Diagnosis: ACR: "ACR criteria were applied by one of the physicians (HHSK) to confirm the diagnosis prior to the enrolment to the study."

Inclusion: 20 to $70 \mathrm{yrs}$ old, have to have had pain VAC $>4 / 10$; using an antidepressants 
Targino 2008 (Continued)

at an analgesic dose (12.5 to $75 \mathrm{mg} / \mathrm{kg}$ ). Author did not specify the name of medication Acupuncture-naive participants: no, patients who had acupuncture in the previous 12 months were excluded. Presumably, this is not an important item because sham acupuncture is not used

Excluded: patients with "severe psychiatric disease, the presence of neurological deficits, cardiac disease or glaucoma, and treatment with acupuncture within one year prior to the start of the study."

Recruitment source: "were recruited by physicians from the Clinics Hospital in Sao Paulo. They included doctors practising in the Pain Clinic of the Department of Neurology, those in the Rheumatology Clinic and those in the Division of Physical Medicine of the Institute of Orthopedics and Traumatology."

Previous treatments: none reported except for current medication

Interventions

1) Real: acupuncture + standard care (12.5 to $75 \mathrm{mg}$ of tricyclic antidepressants per day), individualised plus exercise, including "oral instruction to walk for $30 \mathrm{~min}$ twice a week at their own pace, to breathe deeply and to perform mental relaxation exercises for another $30 \mathrm{~min}$. They were also told to perform twice-weekly stretching exercises involving the para-spinalis muscles, glutei, hamstrings, ankle plantar flexors and hip flexors. "Patients in the acupuncture group always had their sessions performed by the same physician (RAT)

Randomised to this group: 34

2) Control: standard care, tricyclic antidepressant (individualised, ranging from 12.5 to

$75 \mathrm{mg} /$ day, most $(84.5 \%)$ received $50 \mathrm{mg} /$ day) plus exercise. Participants "were seen by a physician at the beginning of the study and during the follow-up visits. No additional visits were scheduled for the controls to compensate for the extra attention being received by patients in the acupuncture treatment group. Compliance with the use of either exercise or antidepressant drugs was based on participants reports during the outcome evaluation interviews."

Randomised to this group: 24

Compliance: $97.1 \%$ (33 participants) completed all 20 sessions, with one leaving after 17 sessions due to complete relief from pain

Minimum number of treatments needed: not reported

Co-intervention: not reported

See STRICTA table for treatment details (Appendix 10)

Outcomes

Primary outcomes:

1) Visual analogue scale (VAS) with $0=$ no pain and $10=$ worst pain experienced

2) Quality of life: SF-36 form. Portuguese version of MOS 36-item short-form health survey (8-items), higher scores indicate better quality of life

Secondary outcomes:

3) Number of tender points below $4 \mathrm{~kg} / \mathrm{cm}^{2}(\mathrm{TePsN})$. The lower the number, the less the severity of symptoms

4) Mean pressure pain threshold value, over the 18 fibromyalgia points (PPT18). The higher the values the less severe the symptoms, measured with algometry (not sure if electronic or manual)

Assessments: at baseline, 3 months (after randomisation and at the end of the treatment) , 6 months, 12 months and 24 months

Outcome measure results:

The 2 groups were comparable at baseline. Also mentioned in the discussion, the usage 
Targino 2008 (Continued)

of medication was not different between the 2 groups

$V A S$ showed statistically significant improvement in the acupuncture group at 3 months $(\mathrm{P}<0.001$, however at 6, 12 and 24 months follow-up, it was not statistically different between the groups $(\mathrm{P}>0.05)$

$S F-36$ showed improvement in only 5 sub-scales of the acupuncture group at 3 months (PF, BP, VT, RE, MH). At 6 months the acupuncture group benefit was for only 1 subscale $(\mathrm{GH})$ and at 12 -month follow-up only 1 sub-scale showed improvement (RP) $T e P s N$ and PPT18 showed improvement in the acupuncture group at 3 and 6 months There was no statistical difference in the scores of the standard care group at any time $(\mathrm{P}>0.05)$

Withdrawals/drop-outs: at 24 months, 2 were lost to follow-up in the acupuncture group (follow-up rate $94.1 \%$ ), 1 in the control group (follow-up rate $95.8 \%$ )

Complications/adverse events: 2 patients in the acupuncture group reported temporary oedema of the left hand at LI4. There were no reported incidences of discomfort, soreness, vasovagal symptoms, bruising or haematoma at time of treatment or the during the follow-up period of 24 months

Data extraction method: data were extracted from the published paper using table 2 and medians/ranges were converted by the CMSG statistician. We could not use SF-36 data as there were no available data for converting median/range to mean. We were waiting for details from the author at the time of publication

Notes

Other info: 1 author of our review was in contact with the study author who that advised she had a paper awaiting publication which she provided direct. This was outside of our search at the start of review and provided further data

Funding: no funding provided

Refunded costs to participants: not reported

Language: English

Publication: full paper

Risk of bias

Bias

Random sequence generation (selection Low risk bias)

Allocation concealment (selection bias)

High risk

Incomplete outcome data (attrition bias) Low risk All outcomes
Authors' judgement

\section{Support for judgement}

Computer-generated random sequence of numbers

Did not use, as study objective was to evaluate the benefit of the addition of acupuncture to a standard course of tricyclic antidepressants and exercise

No drop-out was reported during the treatment or in the first 12 months of follow-up. 3 participants were not contacted at the 24 month follow-up and their data were excluded from analysis at that time. This exclusion will not impact on the outcome at the end of treatment or 12-month followup. Furthermore, the number of drop-outs 
Targino 2008 (Continued)

\begin{tabular}{|c|c|c|}
\hline & & $\begin{array}{l}\text { at } 24 \text { months was comparable between the } \\
2 \text { groups }\end{array}$ \\
\hline Selective reporting (reporting bias) & Low risk & $\begin{array}{l}\text { All outcomes reported as per WHO clinical } \\
\text { trials register }\end{array}$ \\
\hline $\begin{array}{l}\text { Blinding of participants and personnel } \\
\text { (performance bias) } \\
\text { All outcomes }\end{array}$ & High risk & $\begin{array}{l}\text { Participants were restricted to those who } \\
\text { not had acupuncture in the last } 12 \text { months; } \\
\text { care giver was not blinded }\end{array}$ \\
\hline $\begin{array}{l}\text { Blinding of outcome assessment (detection } \\
\text { bias) } \\
\text { All outcomes }\end{array}$ & Low risk & $\begin{array}{l}\text { Outcome assessors were blinded and par- } \\
\text { ticipants were told not to inform them of } \\
\text { their group allocation }\end{array}$ \\
\hline
\end{tabular}

ACR: American College of Rheumatology

CMSG: Cochrane Musculoskeletal Group

FIQ: Fibromyalgia Impact Questionnaire

MPI: Multidimensional Pain Inventory

NCCAM: National Centre for Complementary and Alternative Medicine

$\mathrm{NIH}$ : National Institutes of Health

N/O: non-traditional acupuncture without stimulation

N/S: non-traditional acupuncture with stimulation

PET: positron emission tomography

RCT: randomised controlled trial

SA: non skin-penetrating acupuncture

SD: standard deviation

SE: standard error

SF-MPQ: Short-Form McGill Pain Questionnaire

SNRI: serotonin-norepinephrine reuptake inhibitor

S/S: symptoms/signs

SSRI: selective serotonin re-uptake inhibitor

STRICTA: Standards for Reporting Interventions in Controlled studies of Acupuncture

TA: acupuncture (real)

T/O: traditional acupuncture without stimulation

T/S: traditional acupuncture with stimulation

VAS: visual analogue scale

WHO: World Health Organization 


\begin{tabular}{|c|c|}
\hline Study & Reason for exclusion \\
\hline Cao 2003 & $\begin{array}{l}\text { Article in Chinese. "Randomised" stated but methods not described. Excluded as study had an extra therapy } \\
\text { (mobile cupping) in the acupuncture and Western medicine arm that was not included in the control arm of } \\
\text { Western medicine (i.e. acupuncture + Western medicine + mobile cupping versus Western medicine) }\end{array}$ \\
\hline Cassisi 1994 & $\begin{array}{l}\text { Article in Italian. "Patients were randomly chosen and divided into three therapeutic groups." Translation by } \\
\text { Italian Cochrane Centre confirms article same as Cassisi } 1995 \text { and is a case series. }\end{array}$ \\
\hline Cassisi 1995 & $\begin{array}{l}\text { Article in Italian. "Patients were randomly chosen and divided into three therapeutic groups." Translation by } \\
\text { Italian Cochrane Centre confirms article is a case series. This study has been quoted in a number of reviews } \\
\text { and articles as a RCT but this is incorrect. Contact with one of the authors: the data in this reprint are incorrect } \\
\text { and they advise to use the } 1994 \text { article }\end{array}$ \\
\hline Chen 2009 & Article in Chinese; case series \\
\hline Collazo Chao 2010 & Article in Spanish; not a RCT \\
\hline Dai 2009 & Article in Chinese; case series \\
\hline Feldman 2001 & $\begin{array}{l}\text { Conference report. RCT. The publisher and conference organisers were contacted and could not provide } \\
\text { details of the whereabouts of the authors. Internet searches were undertaken to try and locate either author } \\
\text { without success }\end{array}$ \\
\hline Gong 2010 & $\begin{array}{l}\text { Article in Chinese. Extra therapy not included in both arms (acupuncture }+ \text { mind focus versus Western } \\
\text { medicine) }\end{array}$ \\
\hline Gou 2010 & $\begin{array}{l}\text { Article in Chinese. Extra therapy not included in both arms (acupuncture + infrared lamp versus Western } \\
\text { medicine) }\end{array}$ \\
\hline Guan 2005 & Article in Chinese. Data unusable as responder only. \\
\hline Guevara 2007 & Conference report. No primary clinical outcomes published. \\
\hline Guo 2003 & Article in Chinese. Data unusable as responder only. \\
\hline Guo 2005a & Article in Chinese. Data unusable as responder only (although number of tender points reported for baseline) \\
\hline Harris 2006 & Secondary analysis of original article (Harris 2005) \\
\hline Harris 2007 & Data were from before acupuncture treatment (cross-sectional study) \\
\hline Harris $2007 \mathrm{a}$ & Conference report, RCT. Author was contacted and has advised awaiting full journal publication \\
\hline Harris $2007 \mathrm{~b}$ & Conference report, RCT. Author was contacted and has advised awaiting full journal publication \\
\hline
\end{tabular}


(Continued)

\begin{tabular}{|c|c|}
\hline Jiang 2010 & $\begin{array}{l}\text { Article in Chinese. Invalid control (acupuncture + cupping + Western medicine versus acupuncture + cupping } \\
\text { versus+ Western medicine) }\end{array}$ \\
\hline Lautenschlager 1989 & Article in German. ACR criteria not met. \\
\hline Li 2005 & Article in Chinese. Data unusable as responder only. \\
\hline Li $2005 \mathrm{a}$ & Article in Chinese. Data unusable as responder only. \\
\hline Li 2006 & $\begin{array}{l}\text { Article in Chinese. 'Randomised' stated but methods not described. Excluded as study had an extra therapy } \\
\text { (mobile cupping) in the acupuncture and Western medicine arm that was not included in the control arm of } \\
\text { Western medicine (acupuncture + Western medicine + mobile cupping versus Western medicine) }\end{array}$ \\
\hline Li 2008 & Article in Chinese. Did not report any of our primary outcomes measures \\
\hline Li 2010 & Article in Chinese. Invalid control (acupuncture + moxa versus acupuncture + moxa + Western medicine) \\
\hline Lui 2002 & Article in Chinese. Did not meet ACR criteria. \\
\hline Sandberg 1999 & Swedish study. Not a RCT. \\
\hline Sandberg 2004 & Comparison study. None of the review's primary outcome measures were used \\
\hline Sprott 1995 & $\begin{array}{l}\text { Article in German. Conference report. Author asked that the } 1998 \text { study be considered, although this report } \\
\text { had more data than the } 1998 \text { study }\end{array}$ \\
\hline Sprott 1998 & "Randomly subdivided into 3 groups". Data unusable as shows only 'mean' results \\
\hline Sprott 2000 & Article in German. Unsure how randomised. None of the review's primary outcome measures were used \\
\hline Sun 2008 & Article in Chinese; case series \\
\hline Targino 2002 & RCT; no quantitative data for analyses \\
\hline
\end{tabular}

Uhlemann 2001 Article in German. Randomised. Conference report. Author could not be contacted; no quantitative data for analysis

Wang $2002 \quad$ Article in Chinese. Data unusable as no SD.

Wang $2004 \quad$ Article in Chinese. Data unusable as responder only.

Wei $2006 \quad$ Article in Chinese. Data unusable as responder only.

Wu $2003 \quad$ Article in Chinese. Data unusable as responder only.

Yao $2006 \quad$ Article in Chinese. Data unusable as responder only. 
(Continued)

\begin{tabular}{ll} 
Zhang 2001 & Article in Chinese. Data unusable as responder only. \\
\hline Zhou 2003 & Article in Chinese. Data unusable as responder only. \\
\hline
\end{tabular}

ACR: American College of Rheumatology

FIQ: Fibromyalgia Impact Questionnaire

RCT: randomised controlled trial

SD: standard deviation

\section{Characteristics of ongoing studies [ordered by study ID]}

\section{Vas 2011}

Trial name or title Effects of acupuncture on patients with fibromyalgia: study protocol of a multi-centre randomised controlled trial

\begin{tabular}{ll}
\hline Methods & RCT multi-centre study \\
\hline Participants & 156 participants, aged over 17, ACR diagnosis \\
\hline Interventions & True or sham acupuncture, 9 treatments, once per week \\
\hline Outcomes & FIQ, Hamilton rating scale for depression, medication use. Follow-up 6 and 12 months \\
\hline Starting date & October 2010 to December 2013 \\
\hline Contact information & jorgef.vas.sspa@juntadeandalucia.es \\
\hline Notes &
\end{tabular}

ACR: American College of Rheumatology

FIQ: Fibromyalgia Impact Questionnaire

RCT: randomised controlled trial 
DATA ANDANALYSES

Comparison 1. Acupuncture versus non-acupuncture treatment

\begin{tabular}{|c|c|c|c|c|}
\hline Outcome or subgroup title & $\begin{array}{l}\text { No. of } \\
\text { studies }\end{array}$ & $\begin{array}{c}\text { No. of } \\
\text { participants }\end{array}$ & Statistical method & Effect size \\
\hline $\begin{array}{l}1 \text { Pain up to } 1 \text { month after } \\
\text { treatment }\end{array}$ & 1 & 13 & Mean Difference (IV, Random, 95\% CI) & $-22.4[-40.98,-3.82]$ \\
\hline $\begin{array}{l}2 \text { Global well-being: rated by } \\
\text { participants up to } 1 \text { month } \\
\text { after treatment }\end{array}$ & 1 & 13 & Mean Difference (IV, Random, 95\% CI) & $\begin{array}{l}-15.40[-25.62,-5 . \\
18]\end{array}$ \\
\hline $\begin{array}{l}3 \text { Sleep up to } 1 \text { month after } \\
\text { treatment }\end{array}$ & 1 & 13 & Mean Difference (IV, Random, 95\% CI) & $-0.40[-1.01,0.21]$ \\
\hline $\begin{array}{l}4 \text { Fatigue up to } 1 \text { month after } \\
\text { treatment }\end{array}$ & 1 & 13 & Mean Difference (IV, Random, 95\% CI) & $-1.1[-1.98,-0.22]$ \\
\hline $\begin{array}{l}5 \text { Stiffness up to } 1 \text { month after } \\
\text { treatment }\end{array}$ & 1 & 13 & Mean Difference (IV, Random, 95\% CI) & $-0.90[-1.66,-0.14]$ \\
\hline 6 Adverse events & 1 & 13 & Risk Ratio (M-H, Random, 95\% CI) & $0.0[0.0,0.0]$ \\
\hline $\begin{array}{c}7 \text { Mental well-being up to } 1 \\
\text { month after treatment }\end{array}$ & 1 & 13 & Mean Difference (IV, Random, 95\% CI) & $-0.5[-1.10,0.10]$ \\
\hline
\end{tabular}

Comparison 2. Acupuncture versus placebo or sham acupuncture

\begin{tabular}{|c|c|c|c|c|}
\hline Outcome or subgroup title & $\begin{array}{l}\text { No. of } \\
\text { studies }\end{array}$ & $\begin{array}{c}\text { No. of } \\
\text { participants }\end{array}$ & Statistical method & Effect size \\
\hline $\begin{array}{l}1 \text { Pain (subgroup EA \& MA) up } \\
\text { to } 1 \text { month after treatment }\end{array}$ & 6 & 286 & Std. Mean Difference (IV, Random, 95\% CI) & $-0.14[-0.53,0.25]$ \\
\hline 1.1 Electro-acupuncture & 2 & 104 & Std. Mean Difference (IV, Random, 95\% CI) & $-0.63[-1.02,-0.23]$ \\
\hline 1.2 Manual acupuncture & 4 & 182 & Std. Mean Difference (IV, Random, 95\% CI) & $0.14[-0.17,0.45]$ \\
\hline $\begin{array}{l}2 \text { Pain follow-up to } 7 \text { months after } \\
\text { treatment (subgroup EA vs } \\
\text { MA) }\end{array}$ & 2 & 145 & Std. Mean Difference (IV, Random, 95\% CI) & $-0.12[-0.52,0.28]$ \\
\hline 2.1 Electro-acupuncture & 1 & 49 & Std. Mean Difference (IV, Random, 95\% CI) & $-0.37[-0.93,0.20]$ \\
\hline 2.2 Manual acupuncture & 1 & 96 & Std. Mean Difference (IV, Random, 95\% CI) & $0.05[-0.41,0.51]$ \\
\hline $\begin{array}{l}3 \text { Pain: sham non-invasive } \\
\text { acupuncture (not breaking } \\
\text { skin) vs sham invasive } \\
\text { acupuncture (breaking skin) }\end{array}$ & 6 & & Std. Mean Difference (IV, Random, 95\% CI) & Subtotals only \\
\hline 3.1 Sham breaking skin & 3 & 170 & Std. Mean Difference (IV, Random, 95\% CI) & $-0.21[-0.57,0.15]$ \\
\hline 3.2 Sham not breaking skin & 4 & 116 & Std. Mean Difference (IV, Random, 95\% CI) & $0.04[-0.64,0.71]$ \\
\hline 4 Physical function (SF-36) & 1 & 56 & Mean Difference (IV, Random, 95\% CI) & $-5.80[-10.91,-0.69]$ \\
\hline $\begin{array}{l}5 \text { Global well-being: rated by } \\
\text { participants (subgroup EA } \\
\text { vs MA) up to } 1 \text { month after } \\
\text { treatment }\end{array}$ & 3 & 200 & Std. Mean Difference (IV, Random, 95\% CI) & $0.29[-0.44,1.01]$ \\
\hline
\end{tabular}

Acupuncture for treating fibromyalgia (Review)

Copyright () 2013 The Cochrane Collaboration. Published by John Wiley \& Sons, Ltd. 
5.1 Electro-acupuncture

5.2 Manual acupuncture

6 Global well-being follow-up

to 7 months after treatment

(subgroup EA \& MA)

6.1 Electro-acupuncture

6.2 Manual acupuncture

7 Sleep (subgroup EA \& MA) up

to 1 month after treatment

7.1 Electro-acupuncture

7.2 Manual acupuncture

8 Sleep follow-up to 7 months

after treatment (subgroup EA

\& MA)

8.1 Electro-acupuncture

8.2 Manual acupuncture

9 Fatigue (subgroup EA vs MA)

up to 1 month after treatment

9.1 Electro-acupuncture

9.2 Manual acupuncture

10 Fatigue follow-up to 7 months after treatment (subgroup EA vs MA)

10.1 Electro-acupuncture

10.2 Manual acupuncture

11 Stiffness up to 1 month after treatment

12 Stiffness follow-up to 7 months after treatment

13 Adverse events

14 Mental well-being up to 1 month after treatment

15 Mental well-being follow-up to 7 months

16 Analgesic use (number of tablets per week) up to 1 month after treatment

17 Analgesic use (number of participants taking analgesics up to 1 month after treatment)

18 Tenderness up to 1 month after treatment

18.1 Mean pressure pain threshold $\left(\mathrm{kg} / \mathrm{cm}^{2}\right)$

19 Overall well-being: rated by care giver at end of treatment
Std. Mean Difference (IV, Random, 95\% CI) Std. Mean Difference (IV, Random, 95\% CI)

$0.65[0.26,1.05]$

$-0.40[-0.86,0.06]$

145

Std. Mean Difference (IV, Random, 95\% CI)

$-0.03[-0.87,0.81]$

$0.41[-0.15,0.98]$

$-0.45[-0.91,0.01]$

$0.16[-0.29,0.61]$

Std. Mean Difference (IV, Random, 95\% CI)

Std. Mean Difference (IV, Random, 95\% CI)

Std. Mean Difference (IV, Random, 95\% CI)

$0.40[0.01,0.79]$

$-0.25[-0.71,0.21]$

Std. Mean Difference (IV, Random, 95\% CI)

$-0.09[-0.44,0.26]$

Std. Mean Difference (IV, Random, 95\% CI)

Std. Mean Difference (IV, Random, 95\% CI)

Std. Mean Difference (IV, Random, 95\% CI)

Std. Mean Difference (IV, Random, 95\% CI)

Std. Mean Difference (IV, Random, 95\% CI)

Std. Mean Difference (IV, Random, 95\% CI)

$0.07[-0.49,0.63]$

$-0.20[-0.66,0.26]$

$-0.10[-0.81,0.61]$

$-0.85[-1.44,-0.27]$

$0.26[-0.08,0.61]$

$0.04[-0.52,0.59]$

Std. Mean Difference (IV, Random, 95\% CI)

Std. Mean Difference (IV, Random, 95\% CI)

Std. Mean Difference (IV, Random, 95\% CI)

$-0.27[-0.84,0.29]$

$0.30[-0.16,0.76]$

$-0.45[-0.84,-0.06]$

$-0.30[-1.60,1.00]$

$0.44[0.12,1.63]$

$-1.70[-3.13,-0.27]$

$-1.40[-3.01,0.21]$

$-3.20[-10.20,3.80]$

$0.94[0.66,1.32]$

$0.80[0.02,1.58]$

$0.80[0.02,1.58]$

Mean Difference (IV, Random, 95\% CI)

$2.0[0.81,3.19]$ 


\begin{tabular}{|c|c|c|c|c|}
\hline Outcome or subgroup title & $\begin{array}{l}\text { No. of } \\
\text { studies }\end{array}$ & $\begin{array}{c}\text { No. of } \\
\text { participants }\end{array}$ & Statistical method & Effect size \\
\hline $\begin{array}{l}1 \text { Pain up to } 1 \text { month after } \\
\text { treatment }\end{array}$ & 1 & 38 & Mean Difference (IV, Random, 95\% CI) & $\begin{array}{l}-17.3[-24.13,-10 . \\
47]\end{array}$ \\
\hline 2 Adverse events & 1 & 38 & Risk Ratio (M-H, Random, 95\% CI) & $0.0[0.0,0.0]$ \\
\hline $\begin{array}{l}3 \text { Tenderness up to } 1 \text { month after } \\
\text { treatment }\end{array}$ & 1 & 38 & Mean Difference (IV, Random, 95\% CI) & $-4.00[-6.73,-1.27]$ \\
\hline 3.1 Number of tender points & 1 & 38 & Mean Difference (IV, Random, 95\% CI) & $-4.00[-6.73,-1.27]$ \\
\hline
\end{tabular}

\section{Comparison 4. Acupuncture as an adjunct therapy}

\begin{tabular}{|c|c|c|c|c|}
\hline Outcome or subgroup title & $\begin{array}{l}\text { No. of } \\
\text { studies }\end{array}$ & $\begin{array}{c}\text { No. of } \\
\text { participants }\end{array}$ & Statistical method & Effect size \\
\hline $\begin{array}{l}1 \text { Pain up to } 1 \text { month after } \\
\text { treatment }\end{array}$ & 1 & 58 & Mean Difference (IV, Random, 95\% CI) & $-3.0[-3.90,-2.10]$ \\
\hline $\begin{array}{l}2 \text { Pain up to } 7 \text { months after } \\
\text { treatment }\end{array}$ & 1 & 58 & Mean Difference (IV, Random, 95\% CI) & $-0.5[-1.49,0.49]$ \\
\hline 3 Adverse events & 1 & 58 & Risk Ratio (M-H, Fixed, 95\% CI) & $3.57[0.18,71.21]$ \\
\hline $\begin{array}{l}4 \text { Tenderness - number of tender } \\
\text { points below } \mathrm{kg} / \mathrm{cm}^{2} \text { up to } 1 \\
\text { month after treatment }\end{array}$ & 1 & 58 & Mean Difference (IV, Random, 95\% CI) & $-4.5[-6.20,-2.80]$ \\
\hline $\begin{array}{l}5 \text { Tenderness - number of tender } \\
\text { points below } \mathrm{kg} / \mathrm{cm}^{2} \text { up to } 7 \\
\text { month after treatment }\end{array}$ & 1 & 58 & Mean Difference (IV, Random, 95\% CI) & $-2.0[-3.51,-0.49]$ \\
\hline $\begin{array}{l}6 \text { Tenderness - mean pressure } \\
\text { threshold by pressure algometry } \\
\text { up to } 1 \text { month after treatment }\end{array}$ & 1 & 68 & Mean Difference (IV, Fixed, 95\% CI) & $0.70[0.41,0.99]$ \\
\hline $\begin{array}{l}7 \text { Tenderness - mean pressure } \\
\text { threshold by pressure } \\
\text { algometry, follow up to } 7 \\
\text { months after treatment }\end{array}$ & 1 & 58 & Mean Difference (IV, Random, 95\% CI) & $0.60[0.26,0.94]$ \\
\hline
\end{tabular}

Comparison 5. Deep needling with stimulation (T/S) versus deep needling without stimulation (T/O)

\begin{tabular}{lcccc} 
Outcome or subgroup title & $\begin{array}{c}\text { No. of } \\
\text { studies }\end{array}$ & $\begin{array}{c}\text { No. of } \\
\text { participants }\end{array}$ & Statistical method & Effect size \\
\hline $\begin{array}{l}1 \text { Pain up to 1 month after } \\
\text { treatment }\end{array}$ & 1 & & Mean Difference (IV, Random, 95\% CI) & Subtotals only \\
$\quad \begin{array}{l}1.1 \text { NRS } \\
\begin{array}{l}\text { Physical function (SF-36) up to } \\
1 \text { month after treatment }\end{array}\end{array}$ & 1 & 41 & Mean Difference (IV, Random, 95\% CI) & $0.30[-18.34,18.94]$ \\
\end{tabular}



treatment

Analysis I.I. Comparison I Acupuncture versus non-acupuncture treatment, Outcome I Pain up to I month after treatment.

Review: Acupuncture for treating fibromyalgia

Comparison: I Acupuncture versus non-acupuncture treatment

Outcome: | Pain up to | month after treatment

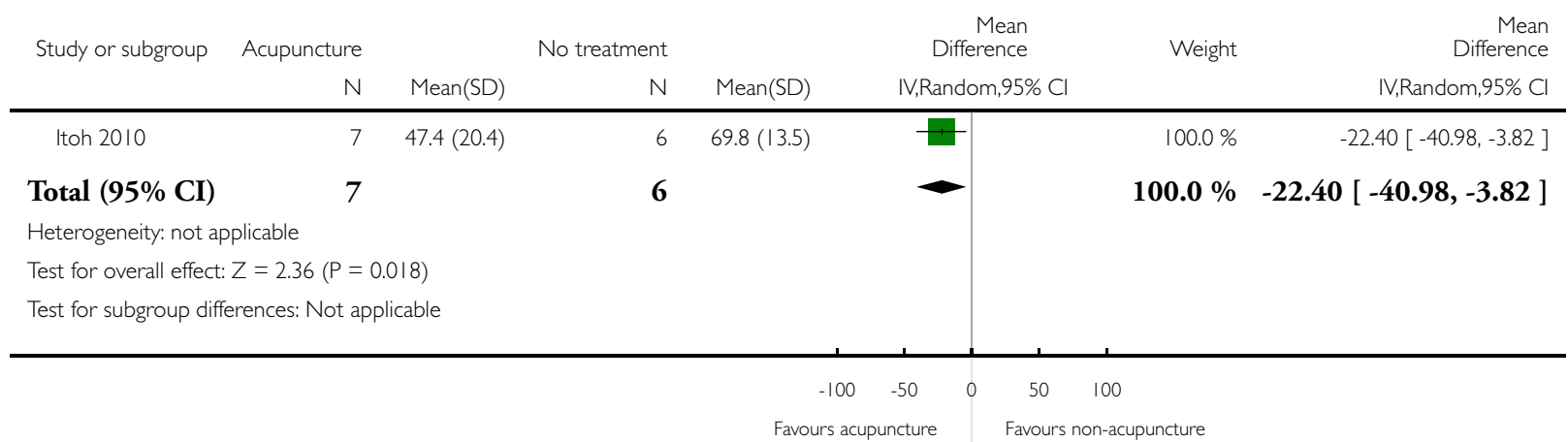


Analysis I.2. Comparison I Acupuncture versus non-acupuncture treatment, Outcome 2 Global well-being: rated by participants up to I month after treatment.

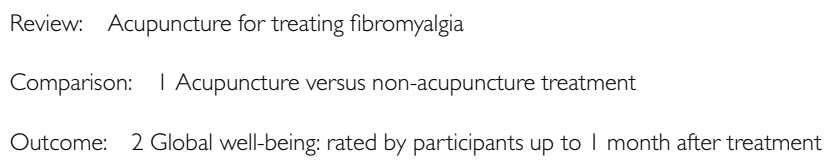

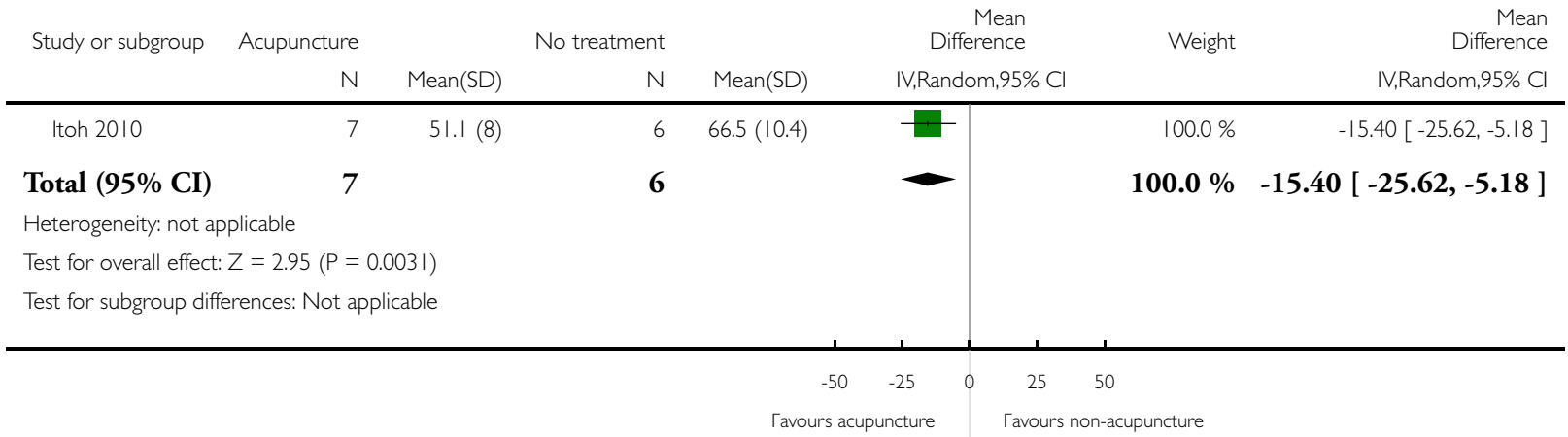

Analysis I.3. Comparison I Acupuncture versus non-acupuncture treatment, Outcome 3 Sleep up to I month after treatment.

Review: Acupuncture for treating fibromyalgia

Comparison: I Acupuncture versus non-acupuncture treatment

Outcome: 3 Sleep up to I month after treatment

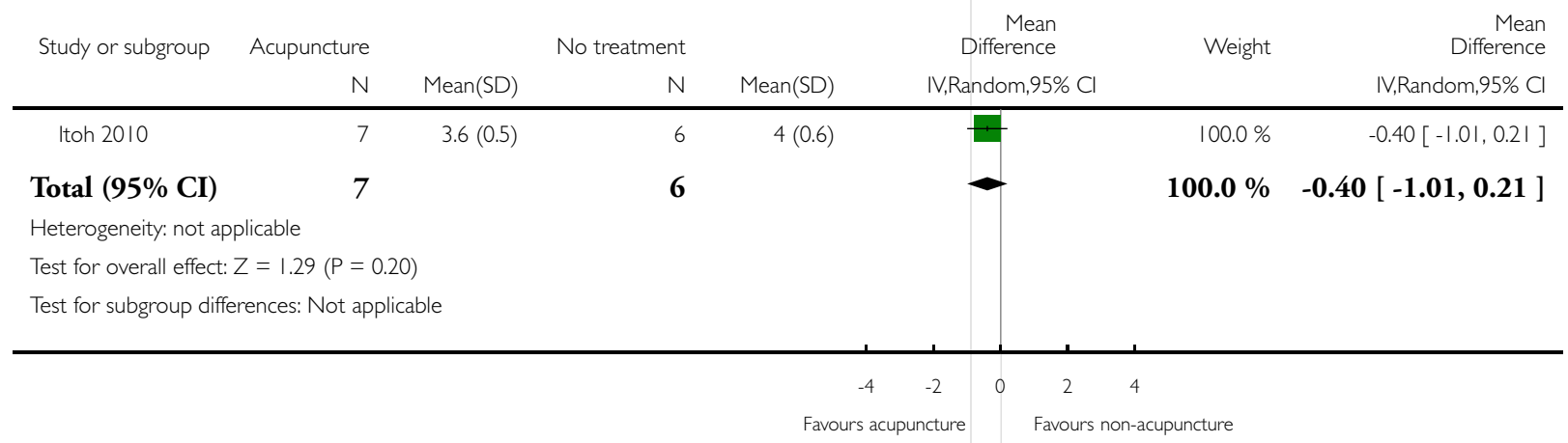


Analysis I.4. Comparison I Acupuncture versus non-acupuncture treatment, Outcome 4 Fatigue up to I month after treatment.

Review: Acupuncture for treating fibromyalgia

Comparison: I Acupuncture versus non-acupuncture treatment

Outcome: 4 Fatigue up to I month after treatment

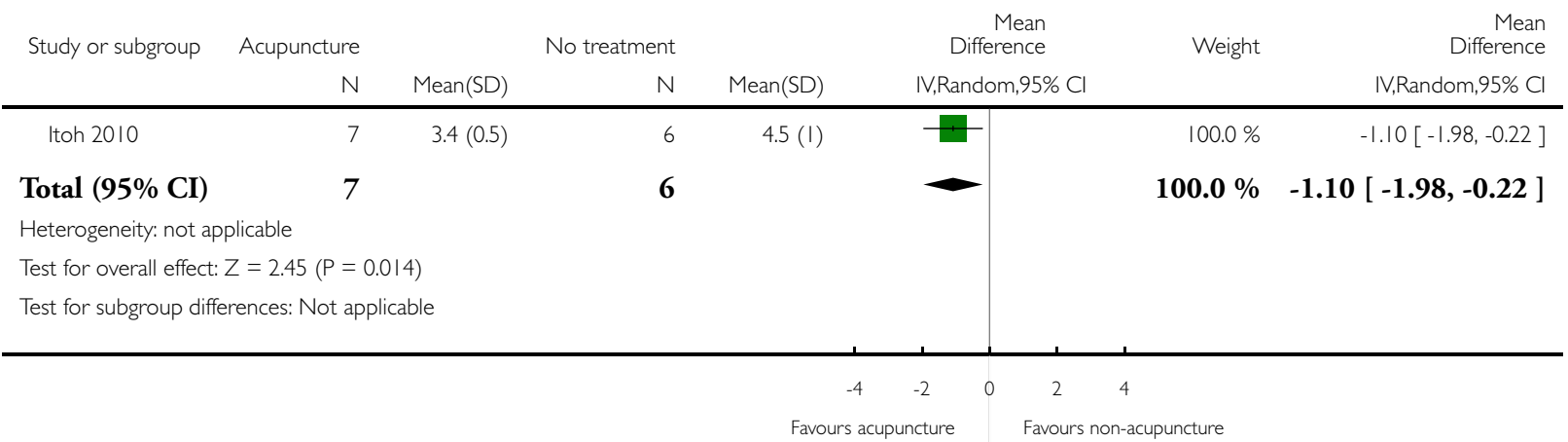

Analysis I.5. Comparison I Acupuncture versus non-acupuncture treatment, Outcome 5 Stiffness up to I month after treatment.

Review: Acupuncture for treating fibromyalgia

Comparison: I Acupuncture versus non-acupuncture treatment

Outcome: 5 Stiffness up to I month after treatment

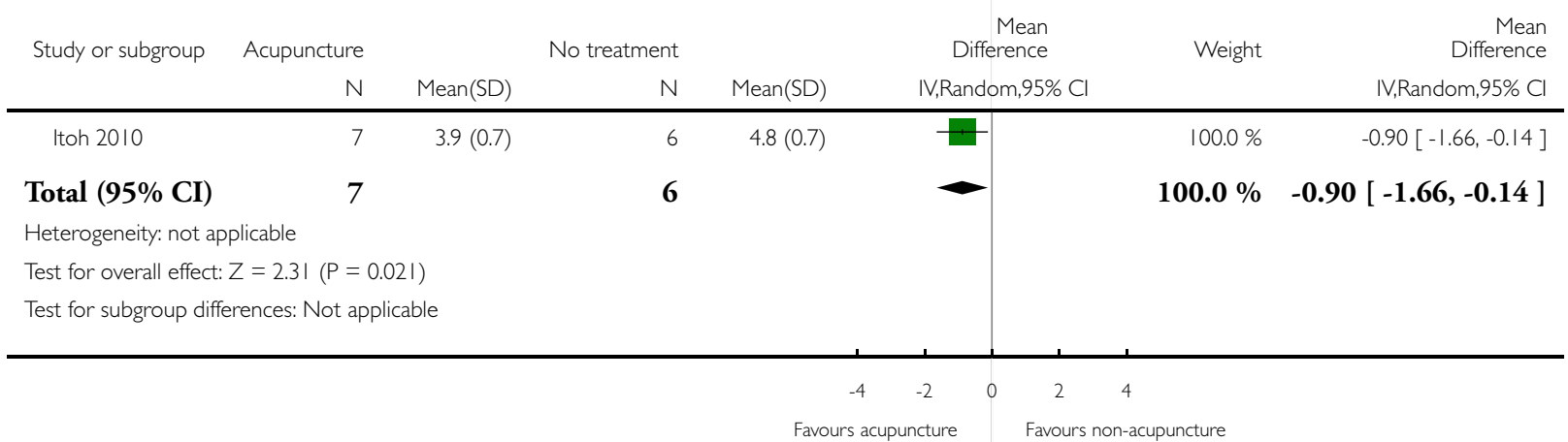


Analysis I.6. Comparison I Acupuncture versus non-acupuncture treatment, Outcome 6 Adverse events.

Review: Acupuncture for treating fibromyalgia

Comparison: I Acupuncture versus non-acupuncture treatment

Outcome: 6 Adverse events

$\mathrm{n} / \mathrm{N} \quad \mathrm{n} / \mathrm{N}$

Itoh 2010

$0 / 7 \quad 0 / 6$

$0 / 6$

Total (95\% CI)

7

6

Risk Ratio
M-

H,Random,95\%

Cl

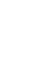

6

Total events: 0 (Acupuncture), 0 (No treatment)

Heterogeneity: not applicable

Test for overall effect: $Z=0.0(P<0.0000$ I $)$

Test for subgroup differences: Not applicable

\section{Analysis I.7. Comparison I Acupuncture versus non-acupuncture treatment, Outcome 7 Mental well-being} up to I month after treatment.

Review: Acupuncture for treating fibromyalgia

Comparison: I Acupuncture versus non-acupuncture treatment

Outcome: 7 Mental well-being up to I month after treatment

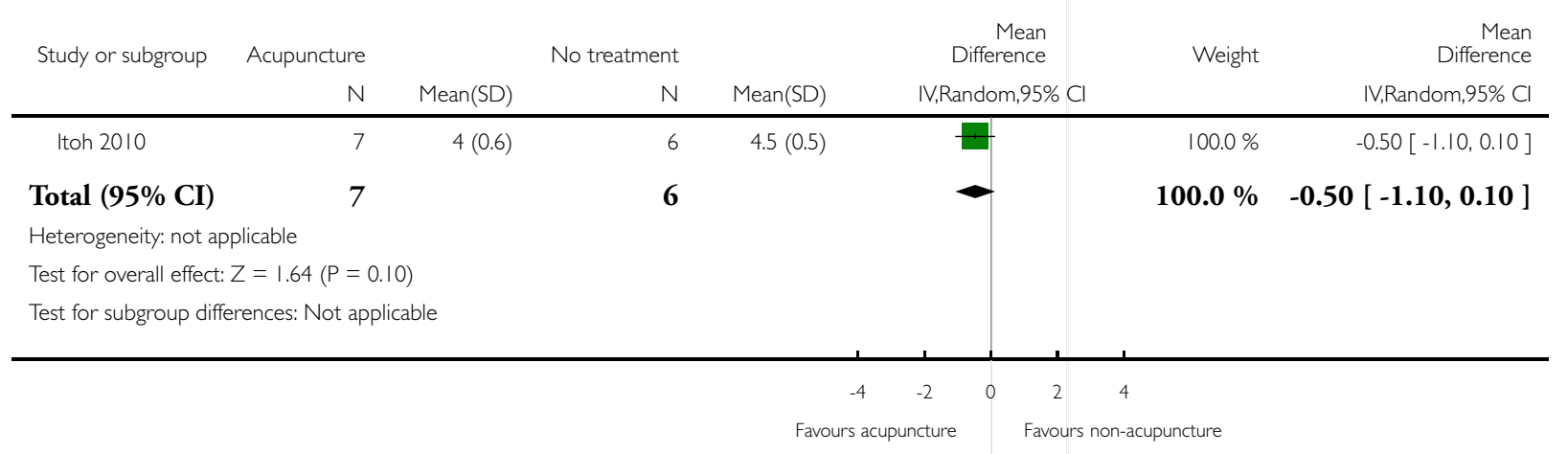


Analysis 2.I. Comparison 2 Acupuncture versus placebo or sham acupuncture, Outcome I Pain (subgroup EA \& MA) up to I month after treatment.

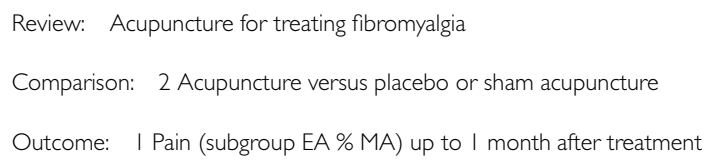

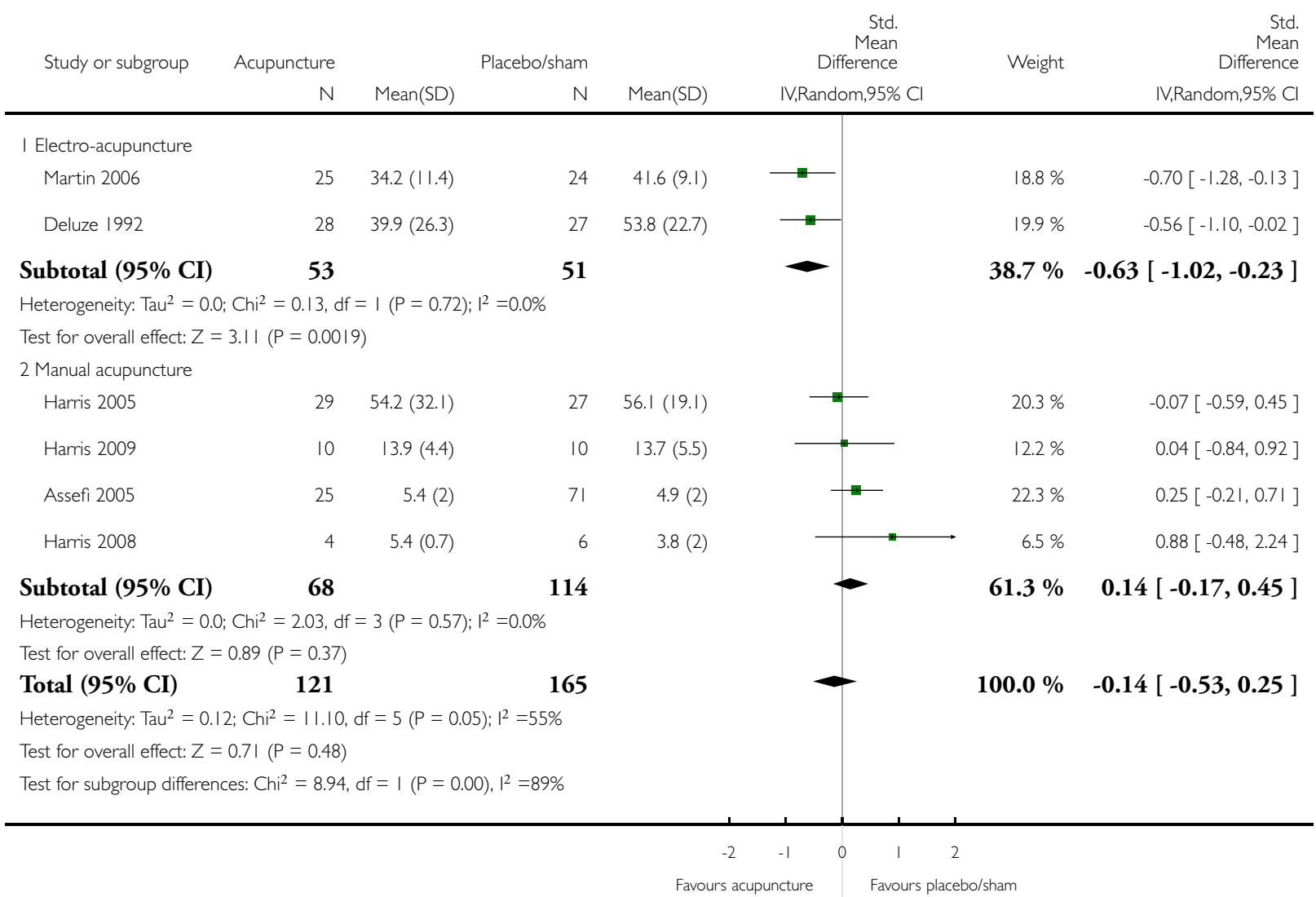


Analysis 2.2. Comparison 2 Acupuncture versus placebo or sham acupuncture, Outcome 2 Pain follow-up to 7 months after treatment (subgroup EA vs MA).

Review: Acupuncture for treating fibromyalgia

Comparison: 2 Acupuncture versus placebo or sham acupuncture

Outcome: 2 Pain follow-up to 7 months after treatment (subgroup EA vs MA)

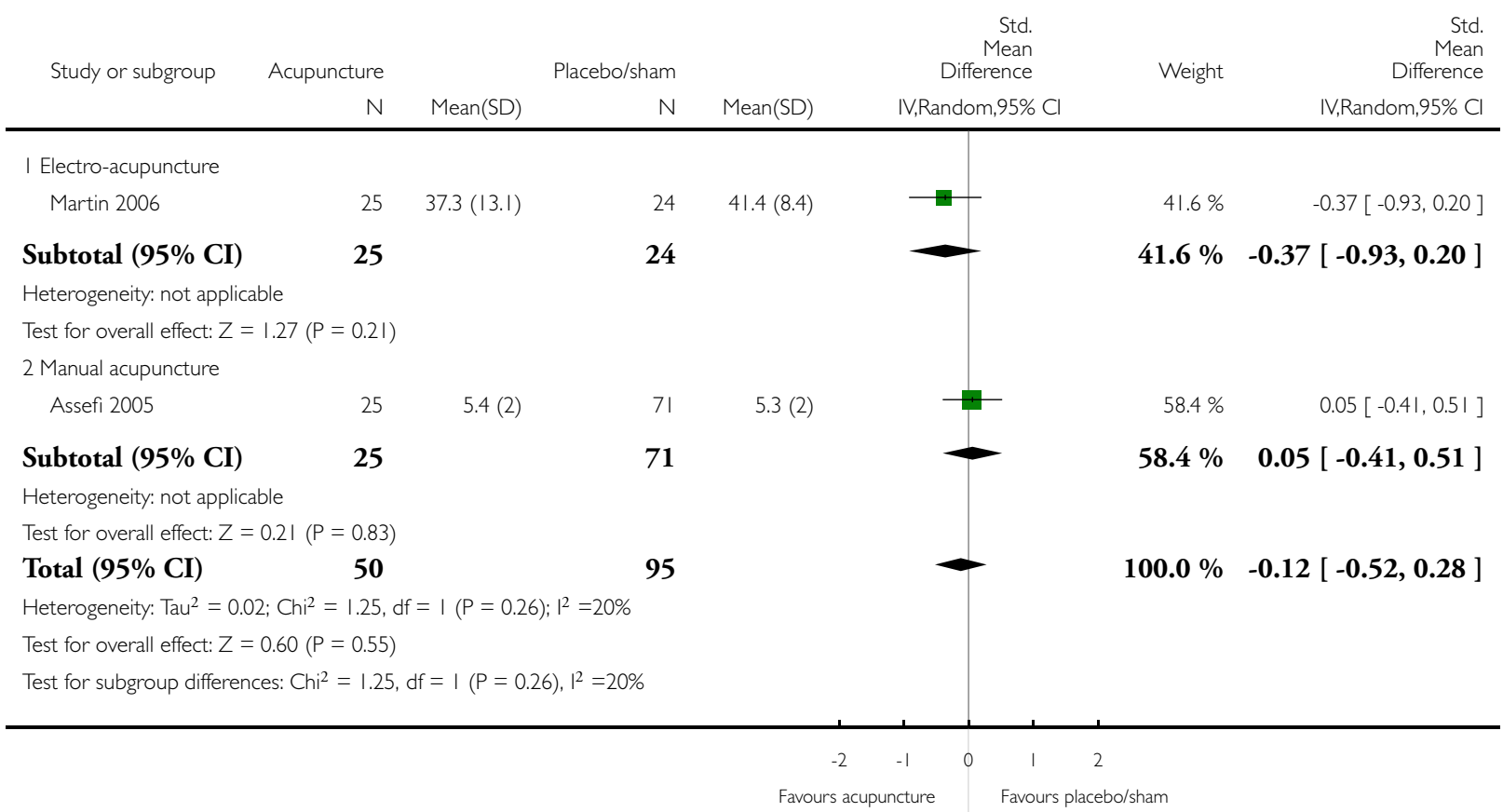


Analysis 2.3. Comparison 2 Acupuncture versus placebo or sham acupuncture, Outcome 3 Pain: sham noninvasive acupuncture (not breaking skin) vs sham invasive acupuncture (breaking skin).

Review: Acupuncture for treating fibromyalgia

Comparison: 2 Acupuncture versus placebo or sham acupuncture

Outcome: 3 Pain: sham non-invasive acupuncture (not breaking skin) vs sham invasive acupuncture (breaking skin)

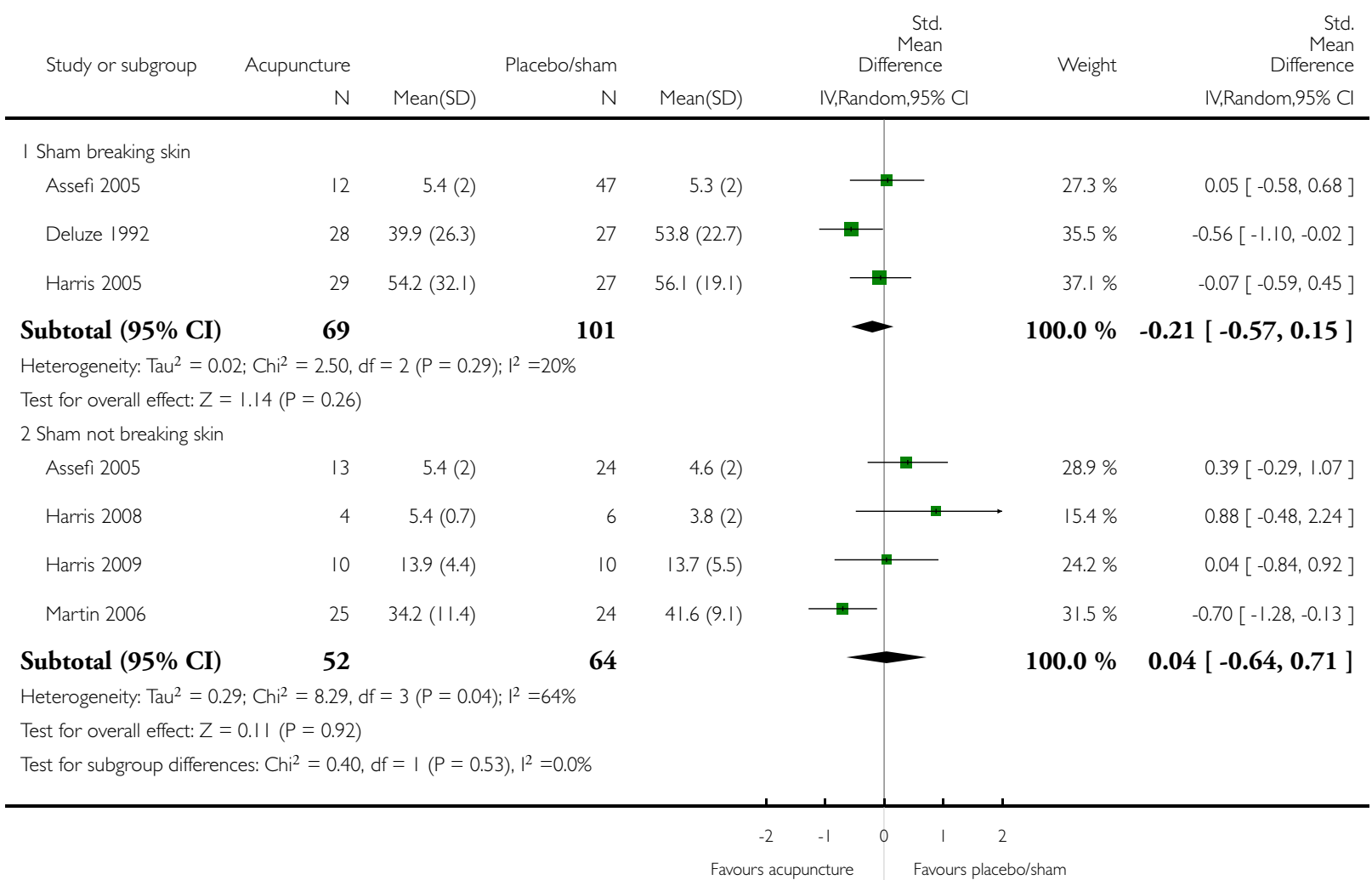


Analysis 2.4. Comparison 2 Acupuncture versus placebo or sham acupuncture, Outcome 4 Physical function (SF-36).

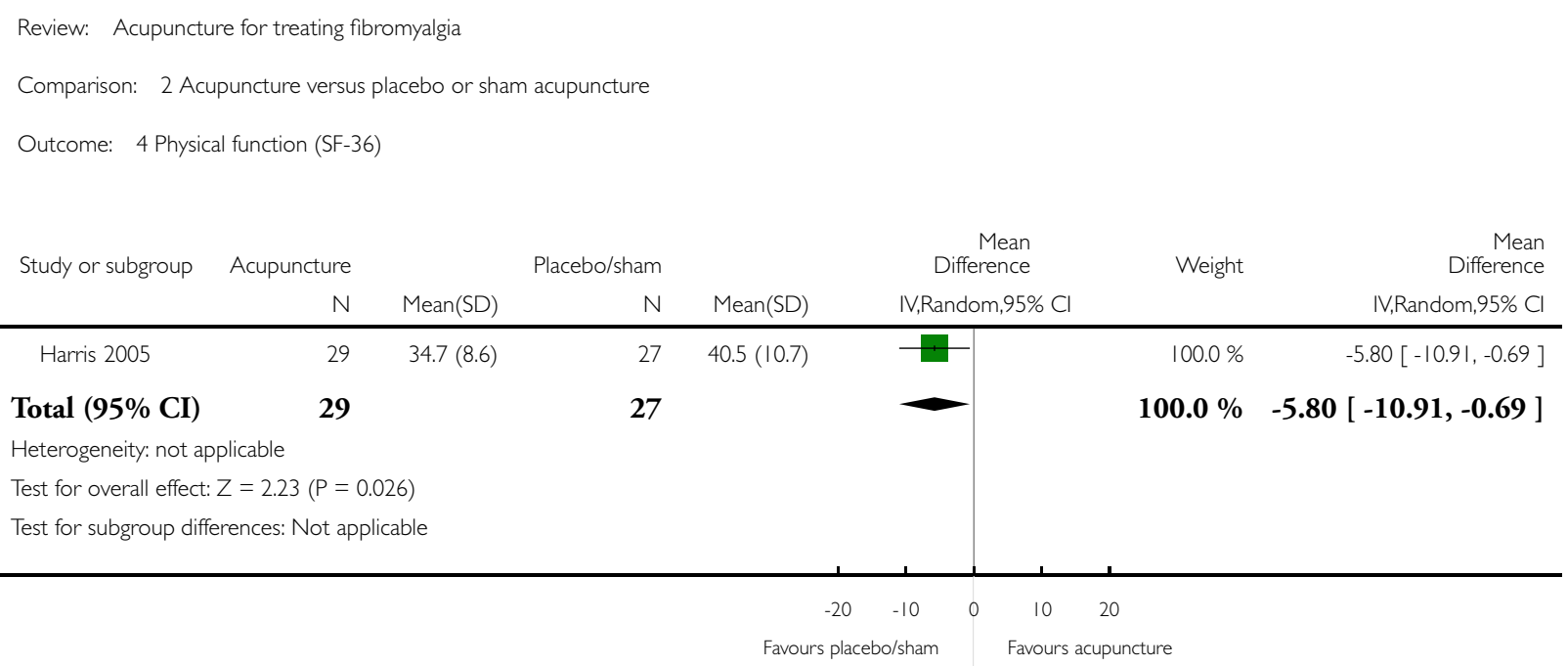


Analysis 2.5. Comparison 2 Acupuncture versus placebo or sham acupuncture, Outcome 5 Global wellbeing: rated by participants (subgroup EA vs MA) up to I month after treatment.

Review: Acupuncture for treating fibromyalgia

Comparison: 2 Acupuncture versus placebo or sham acupuncture

Outcome: 5 Global well-being: rated by participants (subgroup EA vs MA) up to I month after treatment

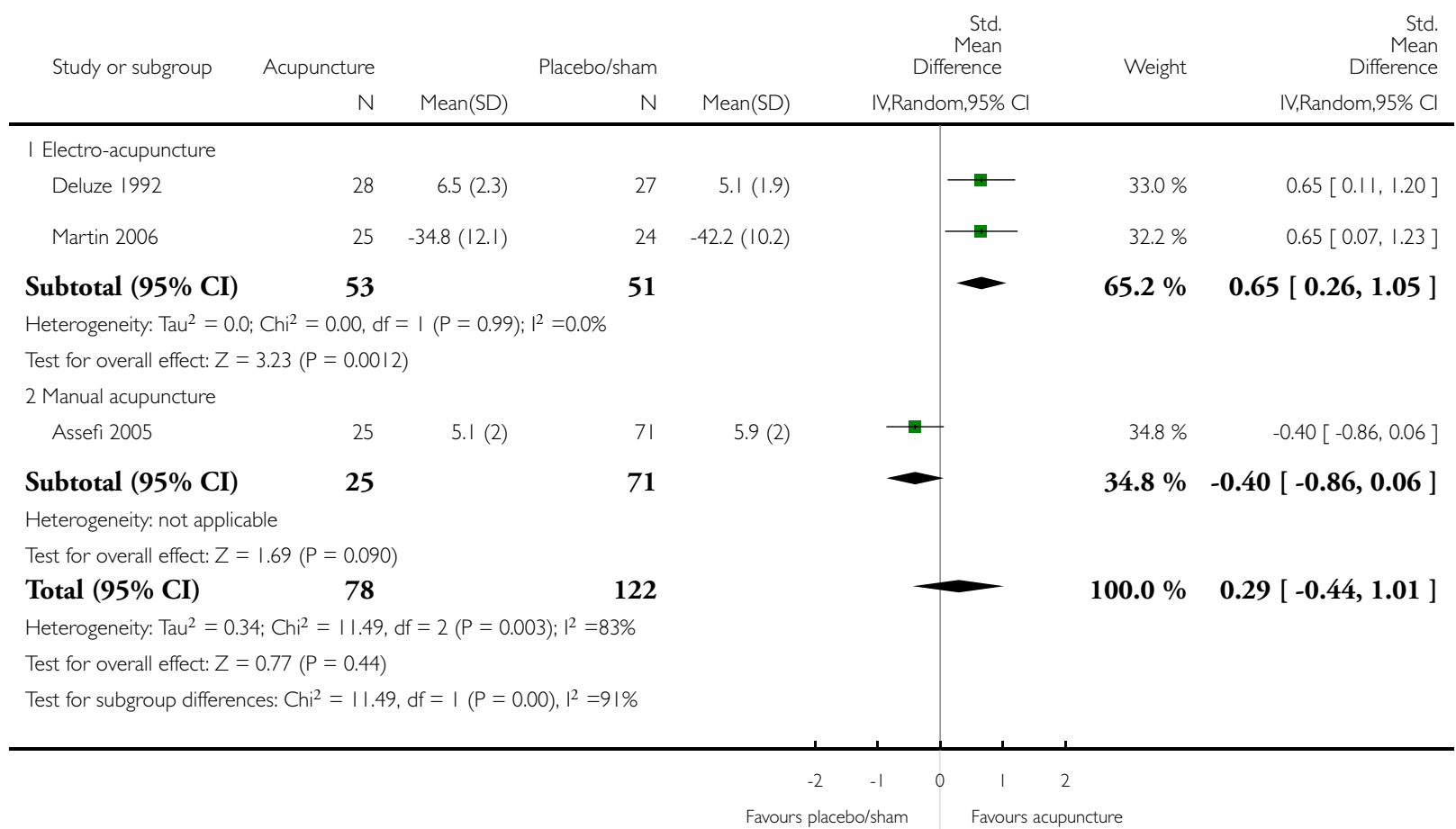


Analysis 2.6. Comparison 2 Acupuncture versus placebo or sham acupuncture, Outcome 6 Global wellbeing follow-up to 7 months after treatment (subgroup EA \& MA).

Review: Acupuncture for treating fibromyalgia

Comparison: 2 Acupuncture versus placebo or sham acupuncture

Outcome: 6 Global well-being follow-up to 7 months after treatment (subgroup EA \% MA)

\begin{tabular}{|c|c|c|c|c|c|c|c|}
\hline \multirow[t]{2}{*}{ Study or subgroup } & Acupuncture & \multicolumn{2}{|c|}{ Placebo/sham } & & \multirow{2}{*}{$\begin{array}{r}\text { Std. } \\
\text { Mean } \\
\text { Difference } \\
\text { IV,Random,95\% Cl }\end{array}$} & \multirow[t]{2}{*}{ Weight } & \multirow{2}{*}{$\begin{array}{r}\text { Std. } \\
\text { Mean } \\
\text { Difference } \\
\text { IV,Random,95\% Cl }\end{array}$} \\
\hline & $\mathrm{N}$ & Mean(SD) & $N$ & Mean(SD) & & & \\
\hline \multicolumn{8}{|l|}{ I Electro-acupuncture } \\
\hline Martin 2006 & 25 & $-38.1(12.1)$ & 24 & $-42.7(9.6)$ & +1 & $48.1 \%$ & $0.41[-0.15,0.98]$ \\
\hline Subtotal $(95 \% \mathrm{CI})$ & 25 & & 24 & & & $48.1 \%$ & $0.41[-0.15,0.98]$ \\
\hline \multicolumn{8}{|c|}{ Heterogeneity: not applicable } \\
\hline \multicolumn{8}{|c|}{ Test for overall effect: $Z=1.43(P=0.15)$} \\
\hline \multicolumn{8}{|l|}{2 Manual acupuncture } \\
\hline Assefi 2005 & 25 & $4.4(2)$ & 71 & $5.3(2)$ & $\longrightarrow$ & $51.9 \%$ & $-0.45[-0.91,0.01]$ \\
\hline Subtotal (95\% CI) & 25 & & 71 & & & $51.9 \%$ & $-0.45[-0.91,0.01]$ \\
\hline \multicolumn{8}{|c|}{ Heterogeneity: not applicable } \\
\hline \multicolumn{8}{|c|}{ Test for overall effect: $Z=1.90(P=0.057)$} \\
\hline Total (95\% CI) & 50 & & 95 & & & $100.0 \%$ & $-0.03[-0.87,0.81]$ \\
\hline \multicolumn{8}{|c|}{ Heterogeneity: $\operatorname{Tau}^{2}=0.30 ; \mathrm{Chi}^{2}=5.33, \mathrm{df}=\mathrm{I}(\mathrm{P}=0.02) ; \mathrm{I}^{2}=81 \%$} \\
\hline \multicolumn{8}{|c|}{ Test for overall effect: $Z=0.08(P=0.94)$} \\
\hline \multicolumn{8}{|c|}{ Test for subgroup differences: $\mathrm{Chi}^{2}=5.33, \mathrm{df}=\mid(P=0.02), \mathrm{I}^{2}=81 \%$} \\
\hline & & & & -2 & -1 & 2 & \\
\hline & & & & Favours pla & Favours & upuncture & \\
\hline
\end{tabular}


Analysis 2.7. Comparison 2 Acupuncture versus placebo or sham acupuncture, Outcome 7 Sleep (subgroup EA \& MA) up to I month after treatment.

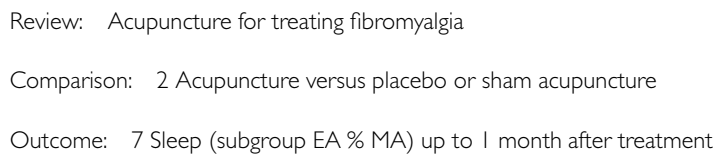

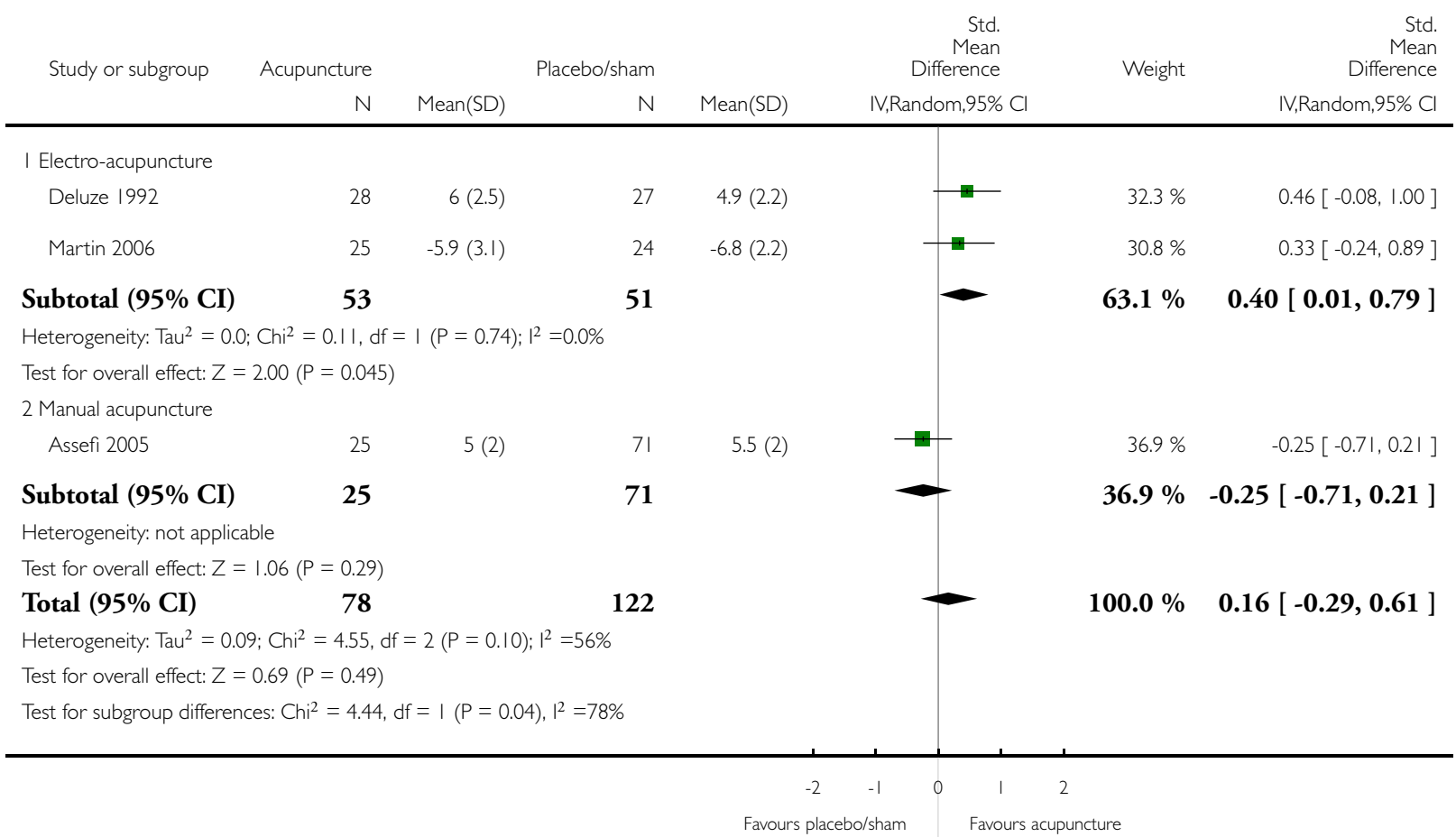


Analysis 2.8. Comparison 2 Acupuncture versus placebo or sham acupuncture, Outcome 8 Sleep follow-up to 7 months after treatment (subgroup EA \& MA).

Review: Acupuncture for treating fibromyalgia

Comparison: 2 Acupuncture versus placebo or sham acupuncture

Outcome: 8 Sleep follow-up to 7 months after treatment (subgroup EA \% MA)

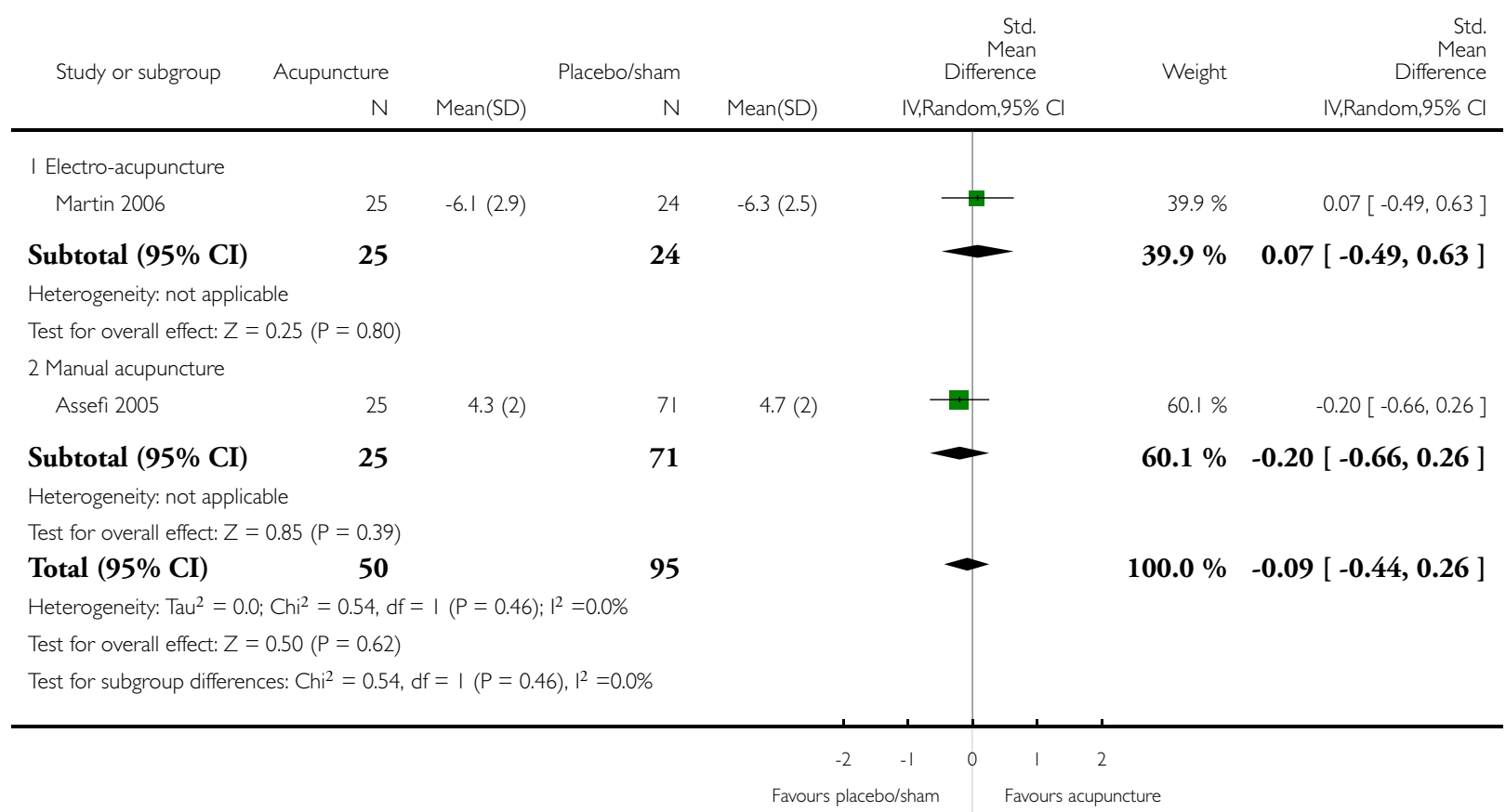


Analysis 2.9. Comparison 2 Acupuncture versus placebo or sham acupuncture, Outcome 9 Fatigue (subgroup EA vs MA) up to I month after treatment.

Review: Acupuncture for treating fibromyalgia

Comparison: 2 Acupuncture versus placebo or sham acupuncture

Outcome: 9 Fatigue (subgroup EA vs MA) up to I month after treatment

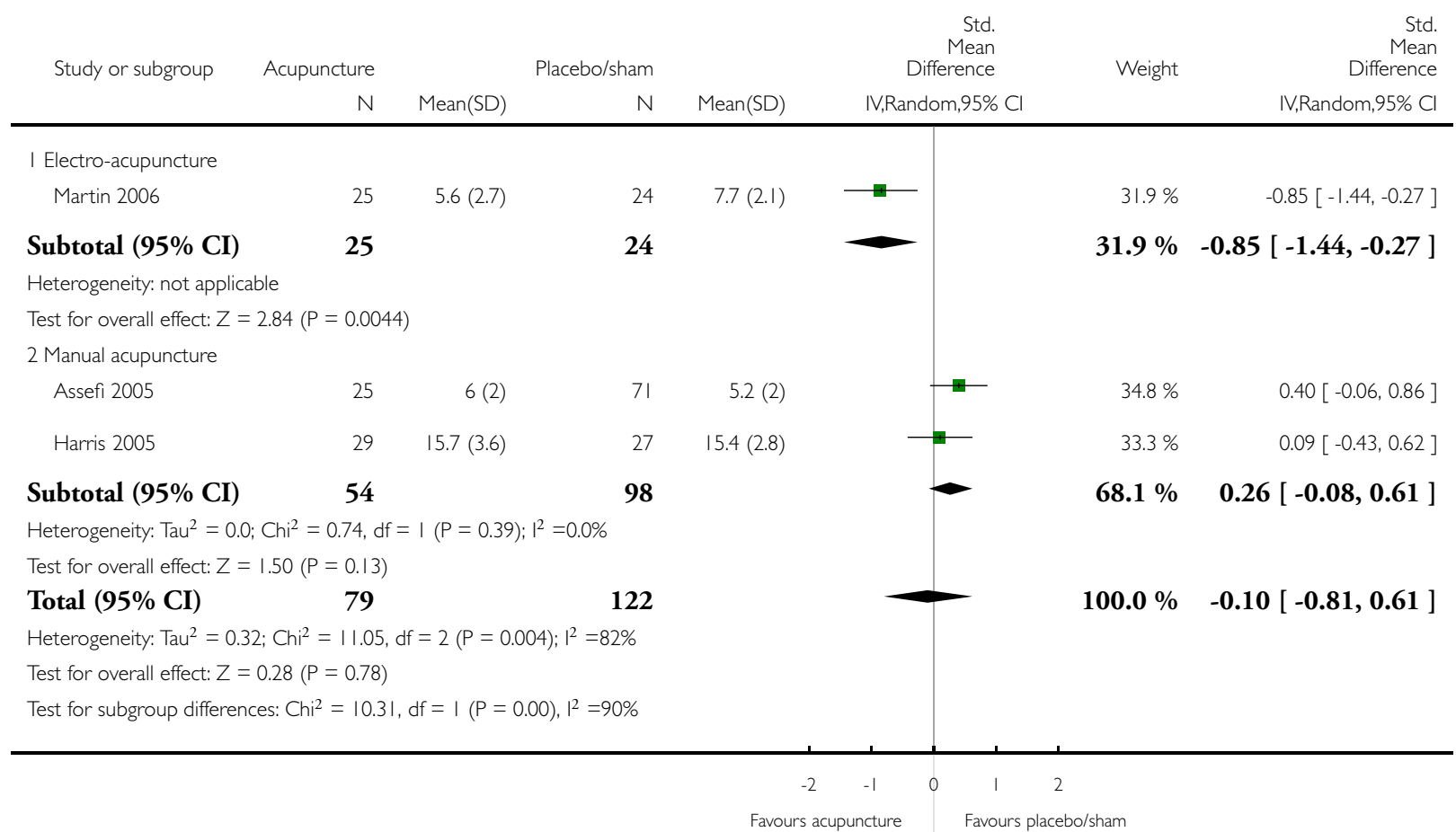


Analysis 2.10. Comparison 2 Acupuncture versus placebo or sham acupuncture, Outcome 10 Fatigue follow-up to 7 months after treatment (subgroup EA vs MA).

Review: Acupuncture for treating fibromyalgia

Comparison: 2 Acupuncture versus placebo or sham acupuncture

Outcome: 10 Fatigue follow-up to 7 months after treatment (subgroup EA vs MA)

\begin{tabular}{|c|c|c|c|c|c|c|c|}
\hline \multirow[t]{2}{*}{ Study or subgroup } & Acupuncture & \multicolumn{2}{|c|}{ Placebo/sham } & & $\begin{array}{r}\text { Std. } \\
\text { Mean } \\
\text { Difference }\end{array}$ & \multirow[t]{2}{*}{ Weight } & \multirow{2}{*}{$\begin{array}{r}\text { Std. } \\
\text { Mean } \\
\text { Difference } \\
\text { IV,Random,95\% Cl }\end{array}$} \\
\hline & $\mathrm{N}$ & Mean(SD) & $\mathrm{N}$ & Mean(SD) & IV,Random,95\% Cl & & \\
\hline \multicolumn{8}{|l|}{ I Electro-acupuncture } \\
\hline Martin 2006 & 25 & $7(2.4)$ & 24 & $7.6(1.9)$ & +1 & $45.7 \%$ & $-0.27[-0.84,0.29]$ \\
\hline Subtotal (95\% CI) & 25 & & 24 & & & $45.7 \%$ & $-0.27[-0.84,0.29]$ \\
\hline \multicolumn{8}{|c|}{ Heterogeneity: not applicable } \\
\hline \multicolumn{8}{|c|}{ Test for overall effect: $Z=0.95(P=0.34)$} \\
\hline \multicolumn{8}{|l|}{2 Manual acupuncture } \\
\hline Assefi 2005 & 25 & $6.1(2)$ & 71 & $5.5(2)$ & 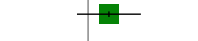 & $54.3 \%$ & $0.30[-0.16,0.76]$ \\
\hline Subtotal (95\% CI) & 25 & & 71 & & & $54.3 \%$ & $0.30[-0.16,0.76]$ \\
\hline \multicolumn{8}{|c|}{ Heterogeneity: not applicable } \\
\hline \multicolumn{8}{|c|}{ Test for overall effect: $Z=1.27(P=0.20)$} \\
\hline Total (95\% CI) & 50 & & 95 & & & $100.0 \%$ & $0.04[-0.52,0.59]$ \\
\hline \multicolumn{8}{|c|}{ Heterogeneity: $\operatorname{Tau}^{2}=0.09 ; \mathrm{Chi}^{2}=2.37, \mathrm{df}=\mathrm{I}(\mathrm{P}=0.12) ; \mathrm{I}^{2}=58 \%$} \\
\hline \multicolumn{8}{|c|}{ Test for overall effect: $Z=0.13(P=0.90)$} \\
\hline \multicolumn{8}{|c|}{ Test for subgroup differences: $\mathrm{Chi}^{2}=2.37, \mathrm{df}=\mathrm{I}(\mathrm{P}=0.12), \mathrm{I}^{2}=58 \%$} \\
\hline & & & & -2 & -1 & 2 & \\
\hline & & & & Favours a & Favours & cebo/sham & \\
\hline
\end{tabular}


Analysis 2.II. Comparison 2 Acupuncture versus placebo or sham acupuncture, Outcome II Stiffness up to I month after treatment.

Review: Acupuncture for treating fibromyalgia

Comparison: 2 Acupuncture versus placebo or sham acupuncture

Outcome: I| Stiffness up to I month after treatment

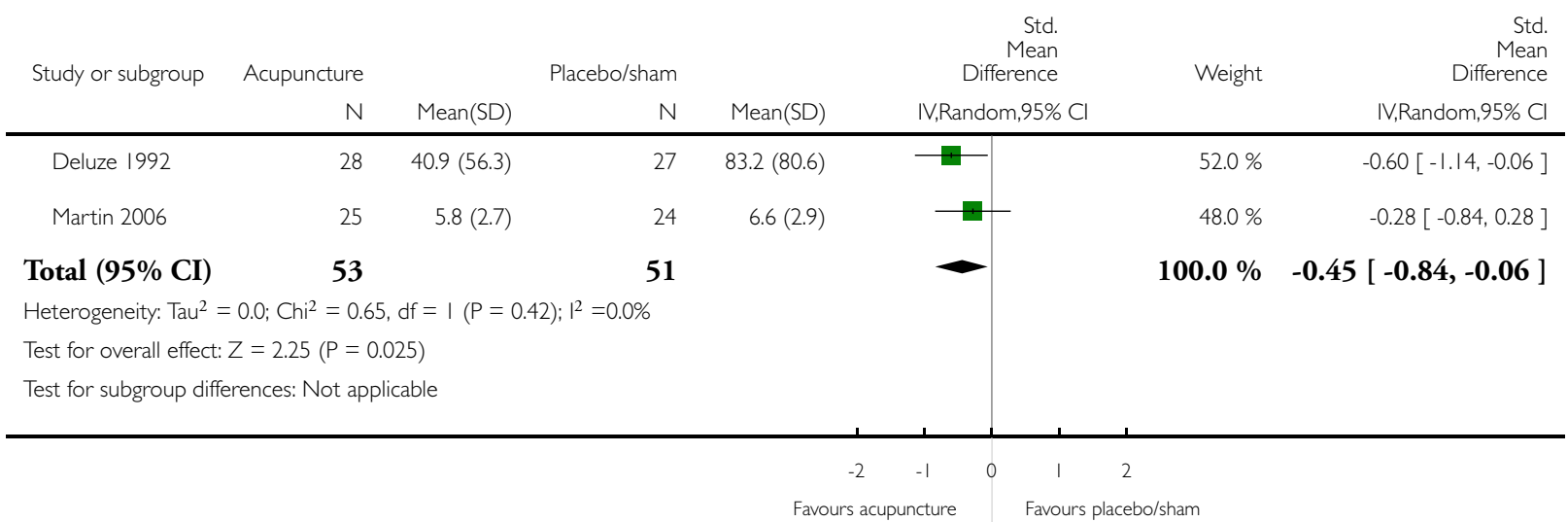

Analysis 2.12. Comparison 2 Acupuncture versus placebo or sham acupuncture, Outcome 12 Stiffness follow-up to 7 months after treatment.

Review: Acupuncture for treating fibromyalgia

Comparison: 2 Acupuncture versus placebo or sham acupuncture

Outcome: 12 Stiffness follow-up to 7 months after treatment

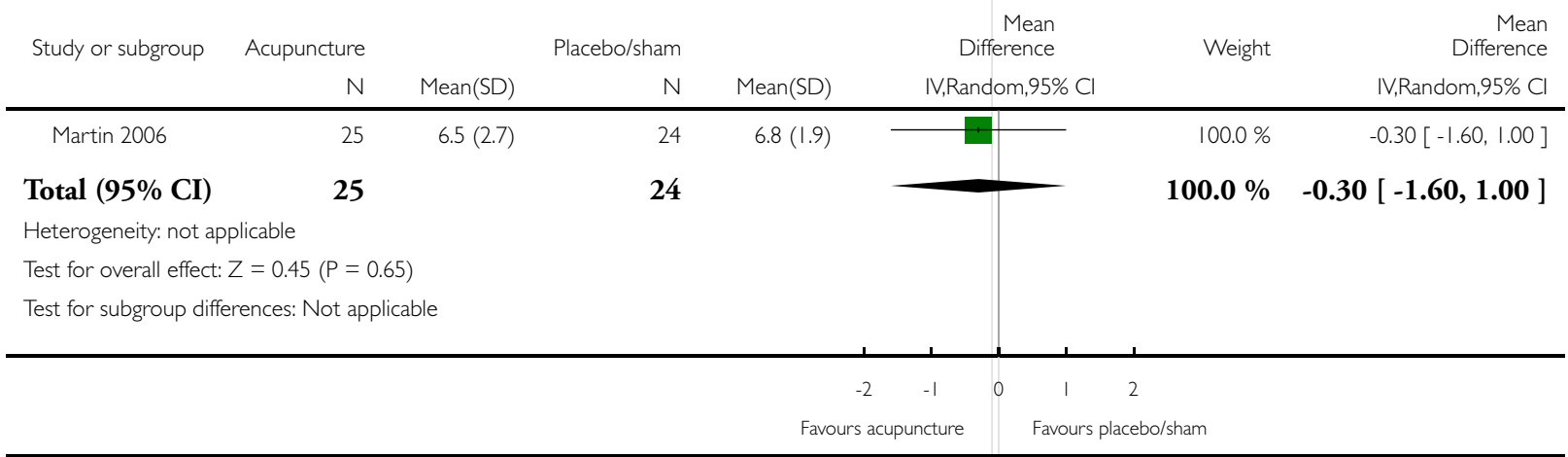

Acupuncture for treating fibromyalgia (Review)

Copyright @ 2013 The Cochrane Collaboration. Published by John Wiley \& Sons, Ltd. 


\section{Analysis 2.13. Comparison 2 Acupuncture versus placebo or sham acupuncture, Outcome 13 Adverse}

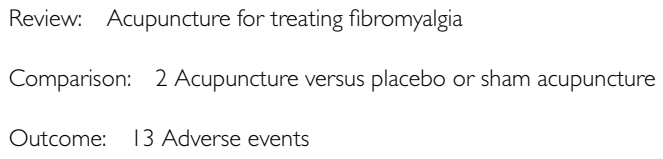

Total events: II (Acupuncture), 58 (Placebo/sham)

Heterogeneity: $\operatorname{Tau}^{2}=0.85 ; \mathrm{Chi}^{2}=6.13, \mathrm{df}=2(\mathrm{P}=0.05) ; \mathrm{I}^{2}=67 \%$

Test for overall effect: $Z=1.23(P=0.22)$

Test for subgroup differences: Not applicable

\section{events.}

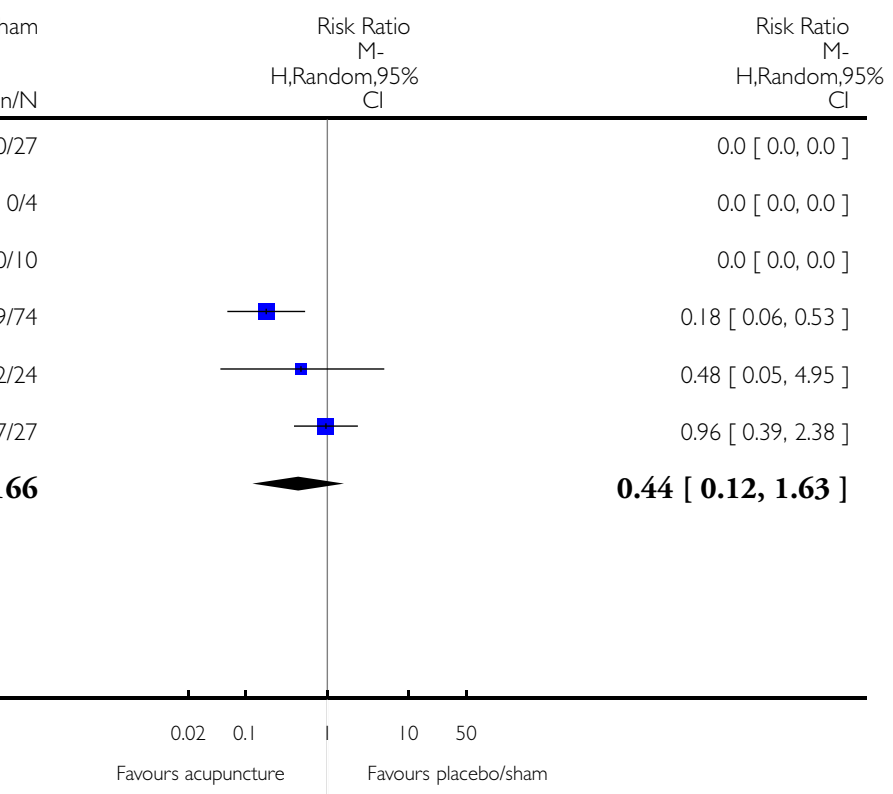


Analysis 2.14. Comparison 2 Acupuncture versus placebo or sham acupuncture, Outcome 14 Mental wellbeing up to I month after treatment.

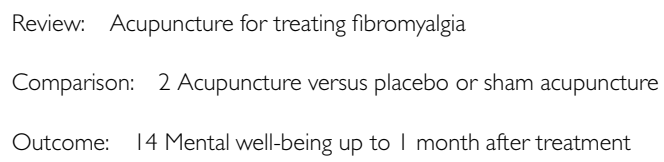

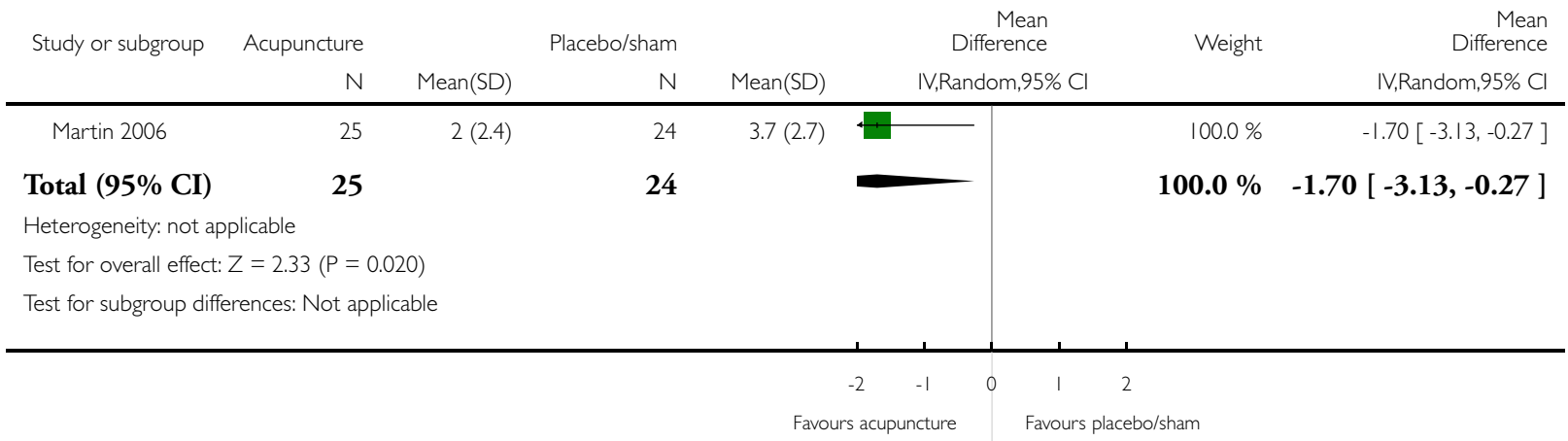

Analysis 2.15. Comparison 2 Acupuncture versus placebo or sham acupuncture, Outcome 15 Mental wellbeing follow-up to 7 months.

Review: Acupuncture for treating fibromyalgia

Comparison: 2 Acupuncture versus placebo or sham acupuncture

Outcome: 15 Mental well-being follow-up to 7 months

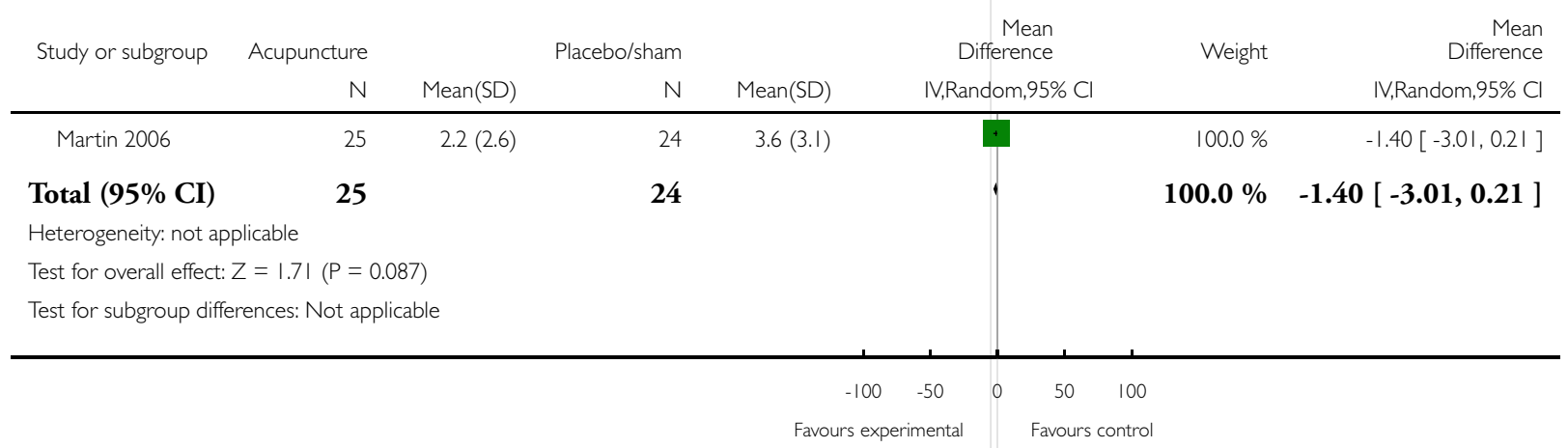


Analysis 2.16. Comparison 2 Acupuncture versus placebo or sham acupuncture, Outcome 16 Analgesic use (number of tablets per week) up to I month after treatment.

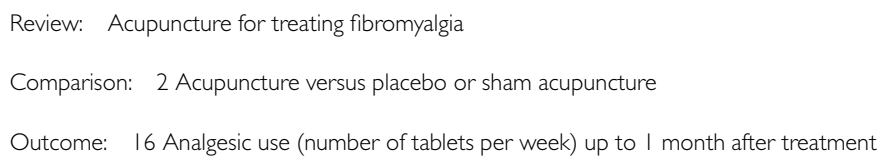

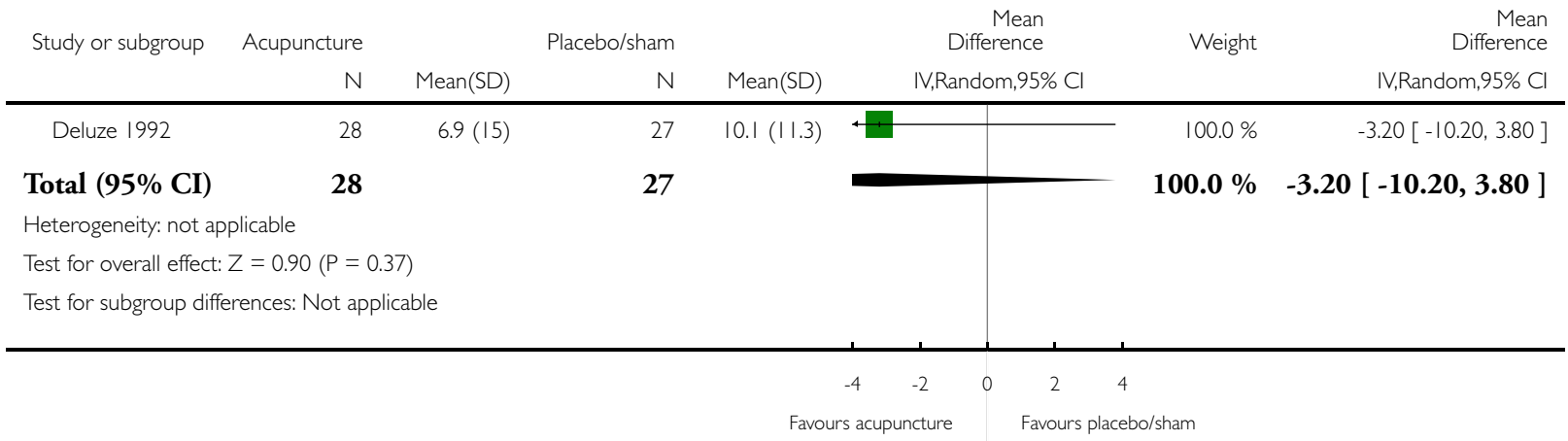

Analysis 2.17. Comparison 2 Acupuncture versus placebo or sham acupuncture, Outcome 17 Analgesic use (number of participants taking analgesics up to I month after treatment).

Review: Acupuncture for treating fibromyalgia

Comparison: 2 Acupuncture versus placebo or sham acupuncture

Outcome: 17 Analgesic use (number of participants taking analgesics up to I month after treatment)

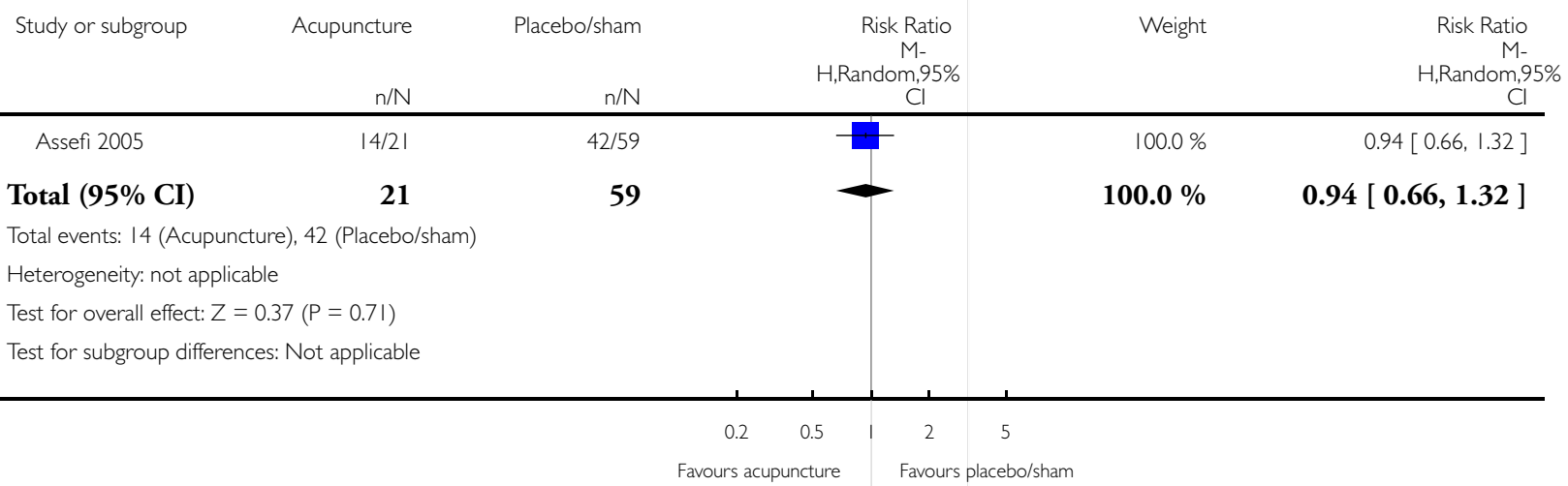


Analysis 2.18. Comparison 2 Acupuncture versus placebo or sham acupuncture, Outcome 18 Tenderness up to I month after treatment.

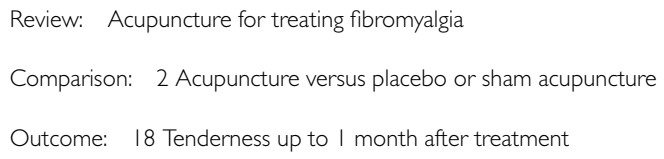

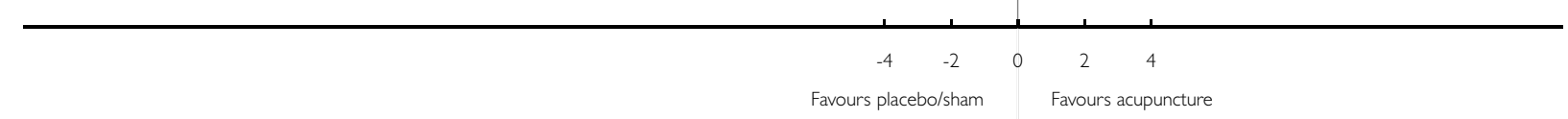

\section{Analysis 2.19. Comparison 2 Acupuncture versus placebo or sham acupuncture, Outcome 19 Overall well-} being: rated by care giver at end of treatment.

Review: Acupuncture for treating fibromyalgia

Comparison: 2 Acupuncture versus placebo or sham acupuncture

Outcome: 19 Overall well-being: rated by care giver at end of treatment

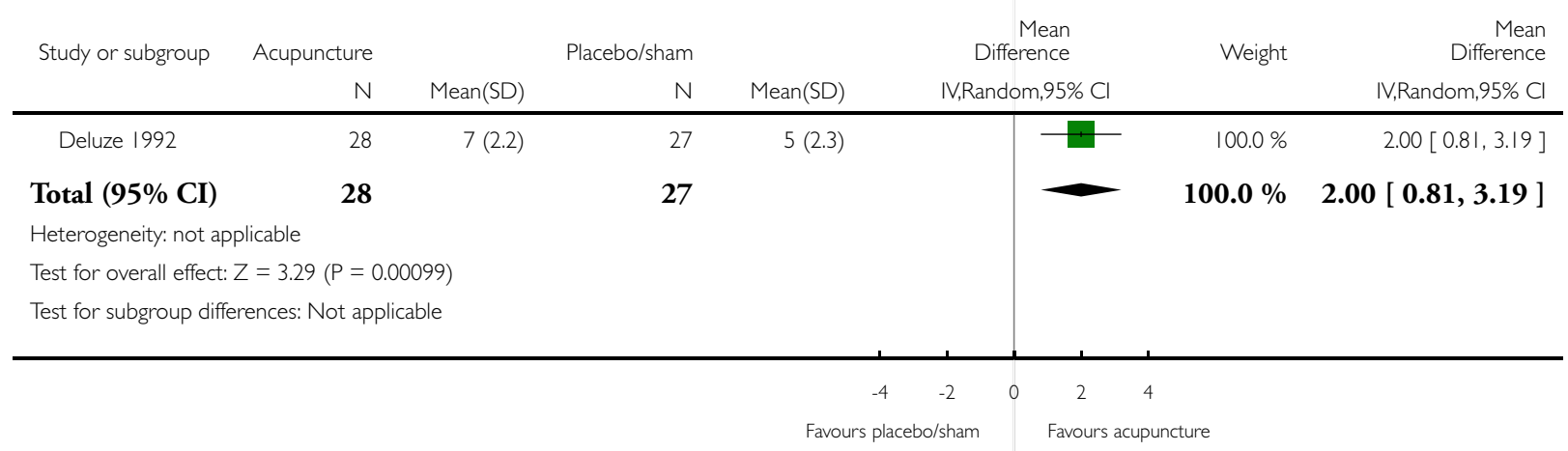


Analysis 3.I. Comparison 3 Acupuncture versus medication, Outcome I Pain up to I month after treatment.

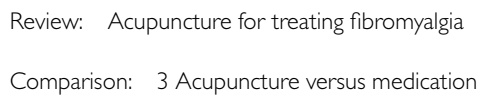

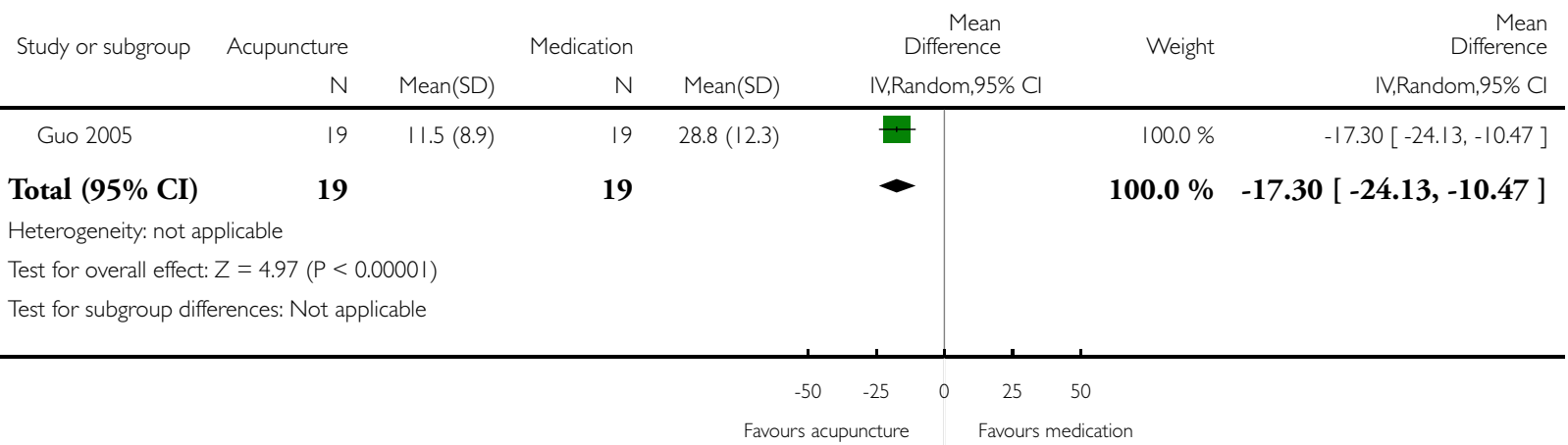

\section{Analysis 3.2. Comparison 3 Acupuncture versus medication, Outcome 2 Adverse events.}

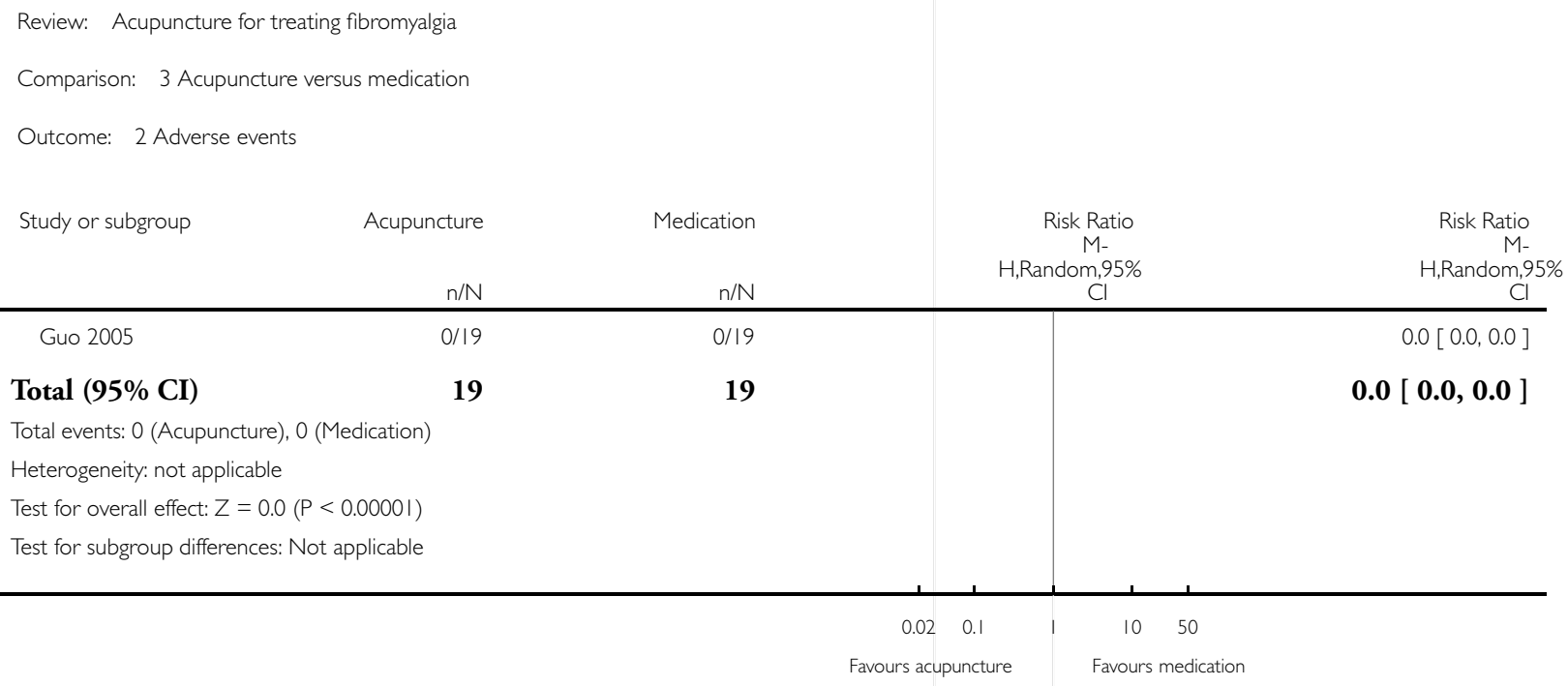


Analysis 3.3. Comparison 3 Acupuncture versus medication, Outcome 3 Tenderness up to I month after treatment.

Review: Acupuncture for treating fibromyalgia

Comparison: 3 Acupuncture versus medication

Outcome: 3 Tenderness up to I month after treatment

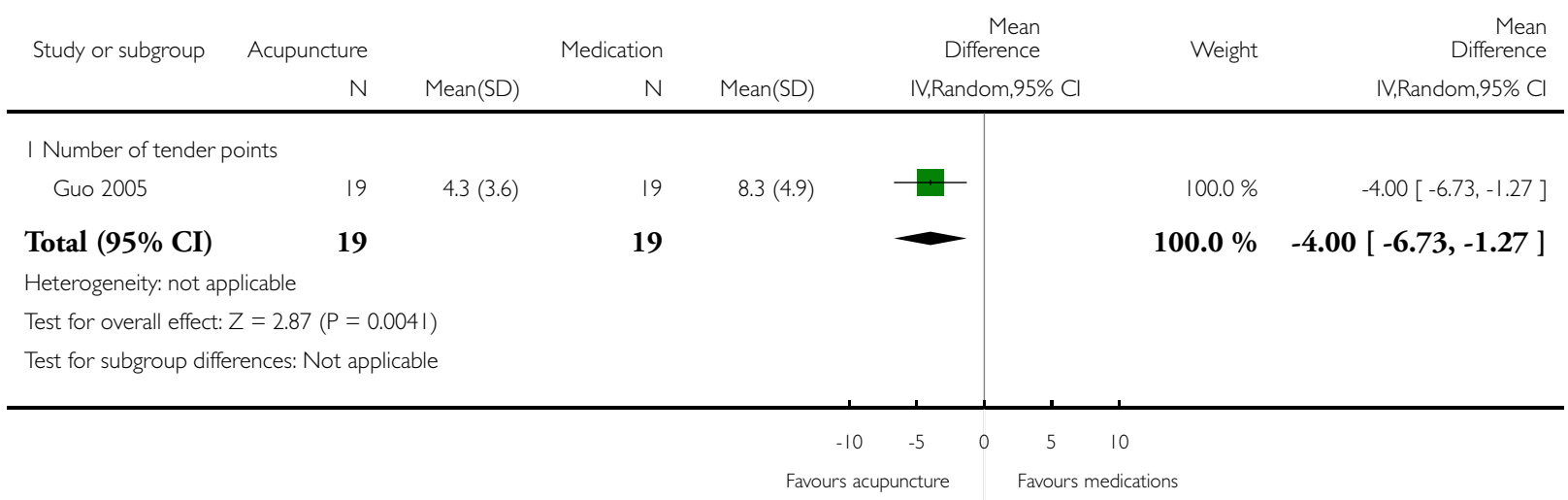

Analysis 4.I. Comparison 4 Acupuncture as an adjunct therapy, Outcome I Pain up to I month after treatment.

Review: Acupuncture for treating fibromyalgia

Comparison: 4 Acupuncture as an adjunct therapy

Outcome: I Pain up to I month after treatment

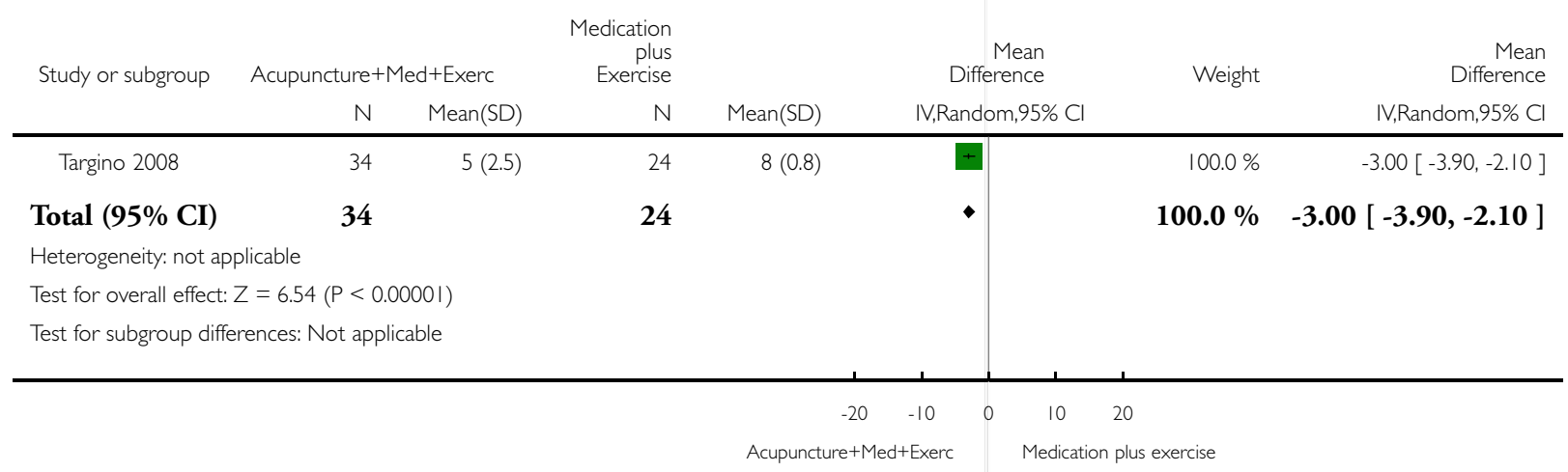


Analysis 4.2. Comparison 4 Acupuncture as an adjunct therapy, Outcome 2 Pain up to 7 months after treatment.

Review: Acupuncture for treating fibromyalgia

Comparison: 4 Acupuncture as an adjunct therapy

Outcome: 2 Pain up to 7 months after treatment

\begin{tabular}{|c|c|c|c|c|c|c|c|}
\hline \multirow[t]{2}{*}{ Study or subgroup } & \multicolumn{3}{|r|}{$\begin{array}{r}\text { Medication } \\
\text { plus } \\
\text { Exercise }\end{array}$} & \multicolumn{2}{|r|}{$\begin{array}{r}\text { Mean } \\
\text { Difference }\end{array}$} & \multirow[t]{2}{*}{ Weight } & \multirow{2}{*}{$\begin{array}{r}\text { Mean } \\
\text { Difference } \\
\text { IV,Random,95\% Cl }\end{array}$} \\
\hline & N & $\operatorname{Mean}(\mathrm{SD})$ & N & $\operatorname{Mean}(\mathrm{SD})$ & IV,Random, $95 \% \mathrm{Cl}$ & & \\
\hline Targino 2008 & 34 & $7(2)$ & 24 & $7.5(1.8)$ & + & $100.0 \%$ & $-0.50[-1.49,0.49]$ \\
\hline Total $(95 \% \mathrm{CI})$ & 34 & & 24 & & $\bullet$ & $100.0 \%$ & $-0.50[-1.49,0.49]$ \\
\hline \multicolumn{8}{|c|}{ Heterogeneity: not applicable } \\
\hline \multicolumn{8}{|c|}{ Test for overall effect: $Z=0.99(P=0.32)$} \\
\hline \multicolumn{8}{|c|}{ Test for subgroup differences: Not applicable } \\
\hline
\end{tabular}

\section{Analysis 4.3. Comparison 4 Acupuncture as an adjunct therapy, Outcome 3 Adverse events.}

Review: Acupuncture for treating fibromyalgia

Comparison: 4 Acupuncture as an adjunct therapy

Outcome: 3 Adverse events

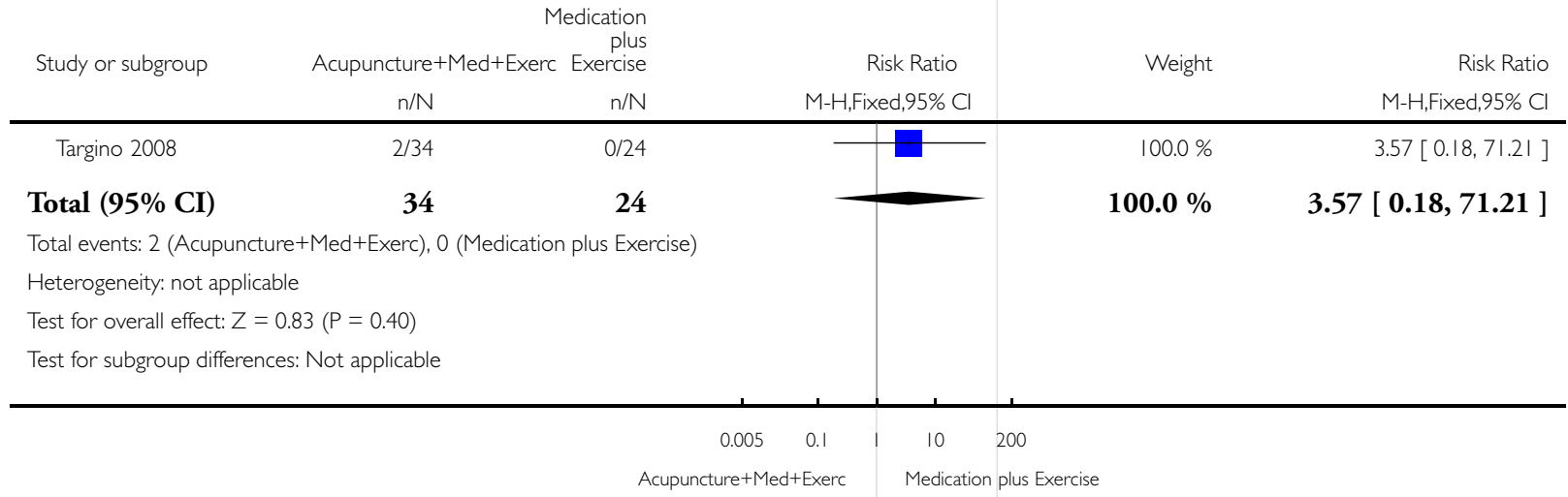


Analysis 4.4. Comparison 4 Acupuncture as an adjunct therapy, Outcome 4 Tenderness - number of tender points below $\mathrm{kg} / \mathrm{cm}^{2}$ up to I month after treatment.

Review: Acupuncture for treating fibromyalgia

Comparison: 4 Acupuncture as an adjunct therapy

Outcome: 4 Tenderness - number of tender points below $\mathrm{kg} / \mathrm{cm}^{2}$ up to I month after treatment

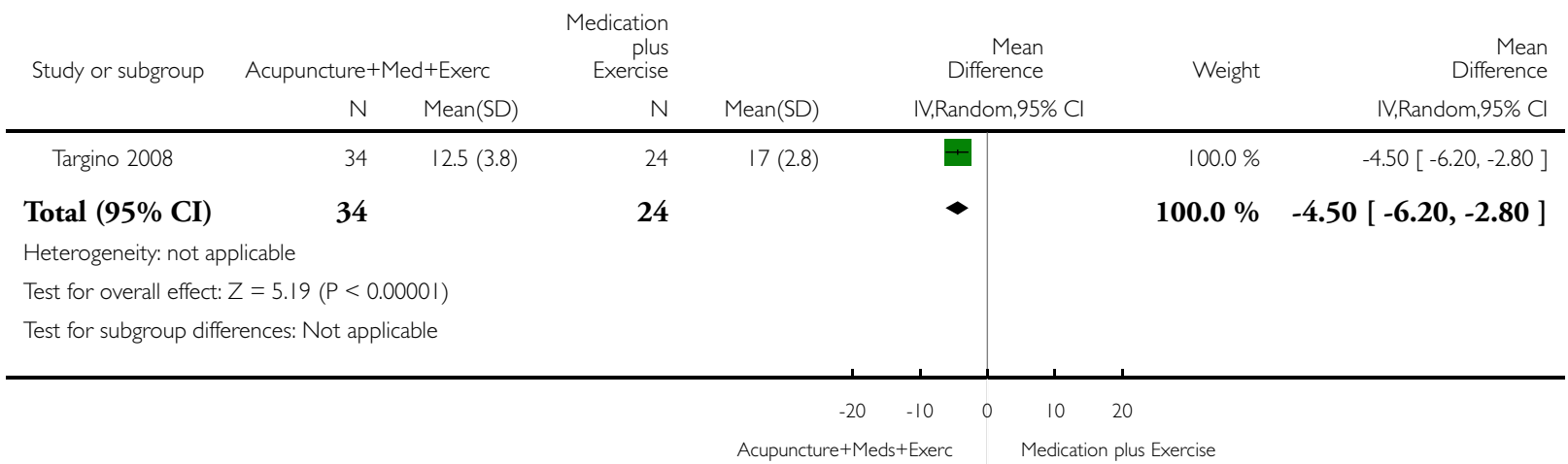

Analysis 4.5. Comparison 4 Acupuncture as an adjunct therapy, Outcome 5 Tenderness - number of tender points below $\mathrm{kg} / \mathrm{cm}^{2}$ up to 7 month after treatment.

Review: Acupuncture for treating fibromyalgia

Comparison: 4 Acupuncture as an adjunct therapy

Outcome: 5 Tenderness - number of tender points below $\mathrm{kg} / \mathrm{cm}^{2}$ up to 7 month after treatment

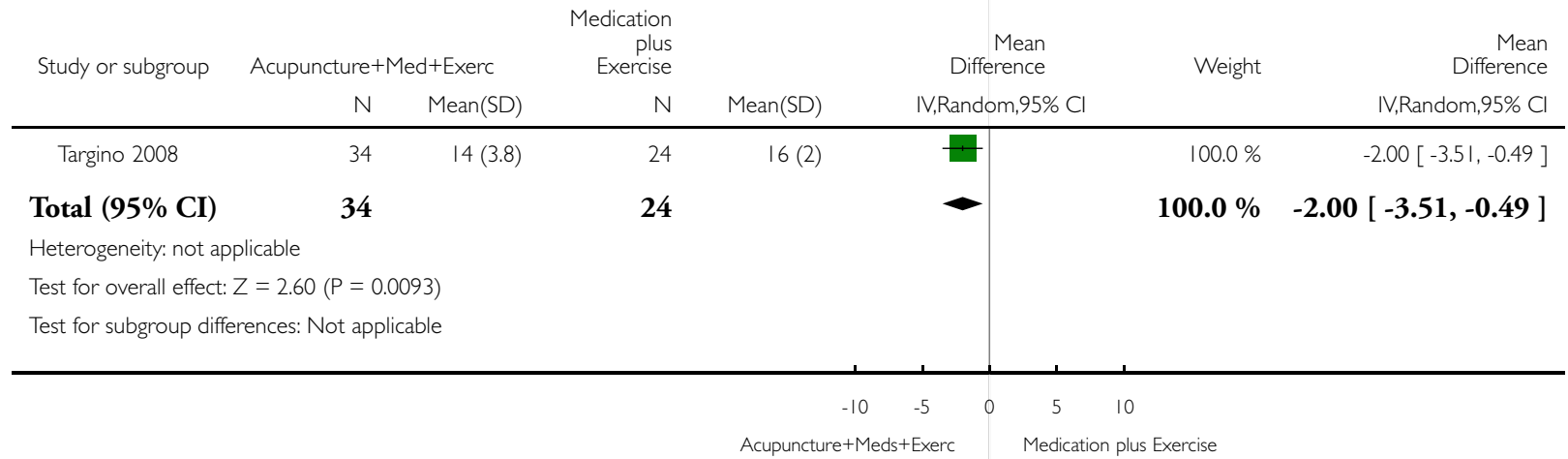

Copyright @ 2013 The Cochrane Collaboration. Published by John Wiley \& Sons, Ltd. 
Analysis 4.6. Comparison 4 Acupuncture as an adjunct therapy, Outcome 6 Tenderness - mean pressure threshold by pressure algometry up to I month after treatment.

Review: Acupuncture for treating fibromyalgia

Comparison: 4 Acupuncture as an adjunct therapy

Outcome: 6 Tenderness - mean pressure threshold by pressure algometry up to I month after treatment

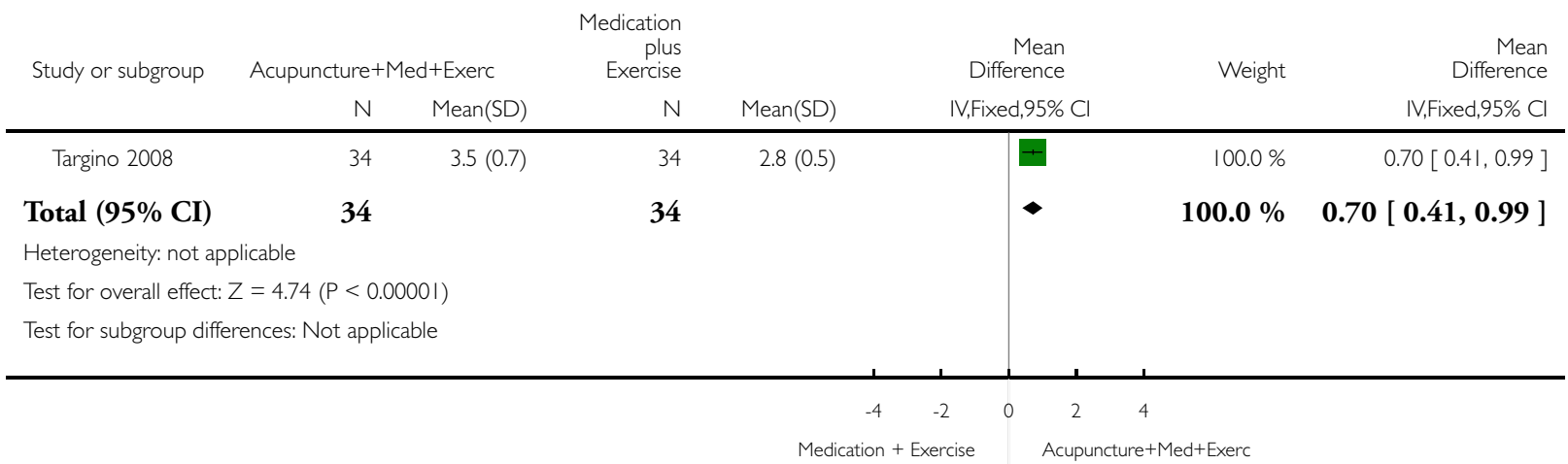

Analysis 4.7. Comparison 4 Acupuncture as an adjunct therapy, Outcome 7 Tenderness - mean pressure threshold by pressure algometry, follow up to 7 months after treatment.

Review: Acupuncture for treating fibromyalgia

Comparison: 4 Acupuncture as an adjunct therapy

Outcome: 7 Tenderness - mean pressure threshold by pressure algometry, follow up to 7 months after treatment

\begin{tabular}{|c|c|c|c|c|c|c|c|c|c|}
\hline \multirow[t]{2}{*}{ Study or subgroup } & \multicolumn{2}{|c|}{ Acupuncture+Med+Exerc } & \multirow{2}{*}{$\begin{array}{r}\text { Medication } \\
\text { plus } \\
\text { Exercise } \\
\mathrm{N}\end{array}$} & \multirow[b]{2}{*}{ Mean(SD) } & & \multirow{2}{*}{\multicolumn{2}{|c|}{$\begin{array}{c}\text { Mean } \\
\text { Difference } \\
\text { IV,Random,95\% Cl }\end{array}$}} & \multirow[t]{2}{*}{ Weight } & \multirow{2}{*}{$\begin{array}{r}\text { Mean } \\
\text { Difference } \\
\text { IV,Random,95\% Cl }\end{array}$} \\
\hline & N & Mean(SD) & & & & & & & \\
\hline Targino 2008 & 34 & $3.5(0.7)$ & 24 & $2.9(0.6)$ & & & + & $100.0 \%$ & $0.60[0.26,0.94]$ \\
\hline Total (95\% CI) & 34 & & 24 & & & & $\bullet$ & $100.0 \%$ & $0.60[0.26,0.94]$ \\
\hline \multicolumn{10}{|c|}{ Heterogeneity: not applicable } \\
\hline \multicolumn{10}{|c|}{ Test for overall effect: $Z=3.50(P=0.00047)$} \\
\hline \multicolumn{10}{|c|}{ Test for subgroup differences: Not applicable } \\
\hline & & & & & -4 & -2 & 2 & 4 & \\
\hline
\end{tabular}

Copyright $@ 2013$ The Cochrane Collaboration. Published by John Wiley \& Sons, Ltd. 
Analysis 5.I. Comparison 5 Deep needling with stimulation (T/S) versus deep needling without stimulation (T/O), Outcome I Pain up to I month after treatment.

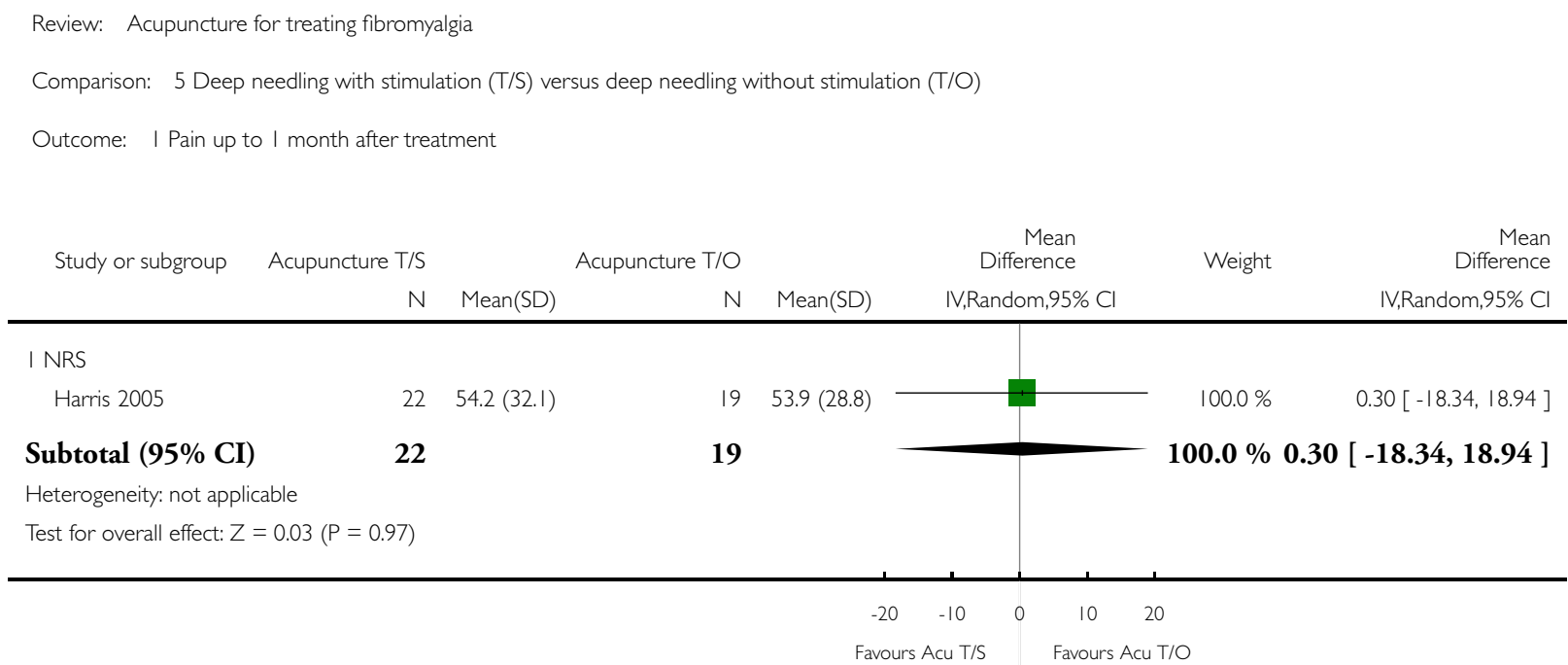

Analysis 5.2. Comparison 5 Deep needling with stimulation (T/S) versus deep needling without stimulation (T/O), Outcome 2 Physical function (SF-36) up to I month after treatment.

Review: Acupuncture for treating fibromyalgia

Comparison: 5 Deep needling with stimulation (T/S) versus deep needling without stimulation (T/O)

Outcome: 2 Physical function (SF-36) up to I month after treatment

\begin{tabular}{|c|c|c|c|c|c|c|c|}
\hline \multirow[t]{2}{*}{ Study or subgroup } & Acupuncture T/S & \multicolumn{2}{|c|}{ Acupuncture $\mathrm{T} / \mathrm{O}$} & \multicolumn{2}{|r|}{$\begin{array}{r}\text { Mean } \\
\text { Difference }\end{array}$} & \multirow[t]{2}{*}{ Weight } & \multirow{2}{*}{$\begin{array}{r}\text { Mean } \\
\text { Difference } \\
\text { IV,Random,95\% Cl }\end{array}$} \\
\hline & $\mathrm{N}$ & Mean(SD) & $\mathrm{N}$ & Mean(SD) & IV,Random,95\% & & \\
\hline Harris 2005 & 22 & $34.7(8.6)$ & 19 & $40.2(10.5)$ & & $100.0 \%$ & $-5.50[-11.43,0.43]$ \\
\hline Total $(95 \% \mathrm{CI})$ & 22 & & 19 & & - & $100.0 \%$ & $-5.50[-11.43,0.43]$ \\
\hline \multicolumn{8}{|c|}{ Heterogeneity: not applicable } \\
\hline \multicolumn{8}{|c|}{ Test for overall effect: $Z=1.82(P=0.069)$} \\
\hline \multicolumn{8}{|c|}{ Test for subgroup differences: Not applicable } \\
\hline
\end{tabular}


Analysis 5.3. Comparison 5 Deep needling with stimulation (T/S) versus deep needling without stimulation (T/O), Outcome 3 Fatigue up to I month after treatment.

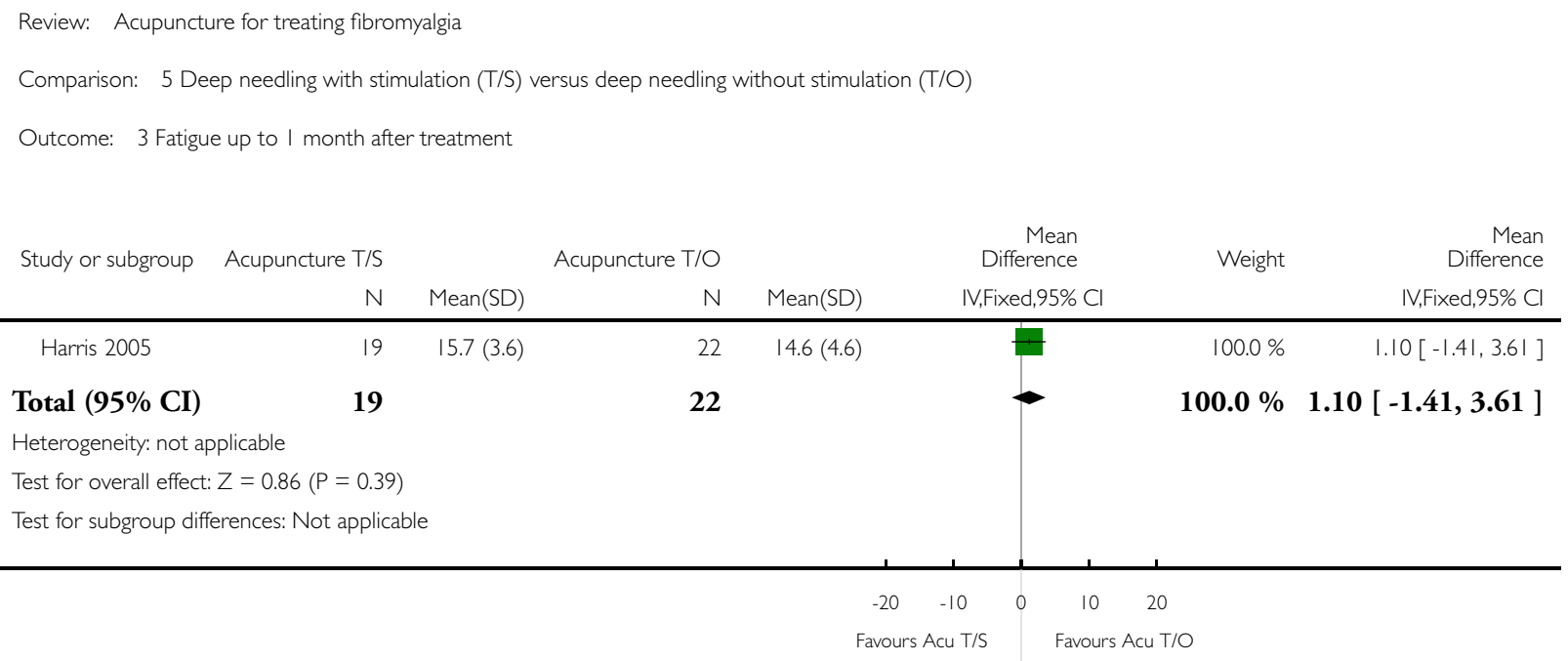

\section{APPENDICES}

\section{Appendix I. Cochrane search strategy and results}

(Updated search on 21 January 2012)

\begin{tabular}{|c|c|c|}
\hline$\# 1$ & MeSH descriptor Fibromyalgia explode all trees & 494 \\
\hline$\# 2$ & (fibromyal*):ti,ab,kw & 790 \\
\hline \#3 & (fibromyalgia syndrome):ti,ab,kw & 275 \\
\hline \#4 & (chronic widespread pain):ti,ab,kw & 52 \\
\hline \#5 & (\#1 OR \#2 OR \#3 OR \#4) & 817 \\
\hline \#6 & (\#5 AND ( randomised AND controlled AND trial )) & 496 \\
\hline \#7 & $\mathrm{MeSH}$ descriptor Acupuncture explode all trees & 127 \\
\hline
\end{tabular}


(Continued)

\begin{tabular}{|c|c|c|}
\hline \#8 & MeSH descriptor Acupuncture Therapy explode all trees & 2470 \\
\hline \#9 & (acupuncture point):ti,ab,kw & 1628 \\
\hline$\# 10$ & (body acupuncture):ti,ab,kw & 242 \\
\hline$\# 11$ & MeSH descriptor Electroacupuncture explode all trees & 381 \\
\hline \#12 & (electro-acupuncture):ti,ab,kw & 191 \\
\hline \#13 & MeSH descriptor Acupuncture, Ear explode all trees & 91 \\
\hline \#14 & (auricular acupuncture):ti,ab,kw & 166 \\
\hline \#15 & (scalp acupuncture):ti,ab,kw & 160 \\
\hline \#16 & (dry needling):ti,ab,kw & 71 \\
\hline \#17 & (trigger point):ti,ab,kw & 360 \\
\hline \#18 & (acupoint injection):ti,ab,kw & 119 \\
\hline \#19 & $\begin{array}{l}\text { (\#7 OR \#8 OR \#9 OR \#10 OR \#11 OR \#12 OR \#13 OR } \\
\# 14\end{array}$ & 3655 \\
\hline \#20 & (\#19 AND ( randomised AND controlled AND trial )) & 2770 \\
\hline \#21 & (\#6 AND \#20) & 28 \\
\hline
\end{tabular}

\section{Appendix 2. MEDLINE search strategy and results}

(Updated searched on 21 January 2012)

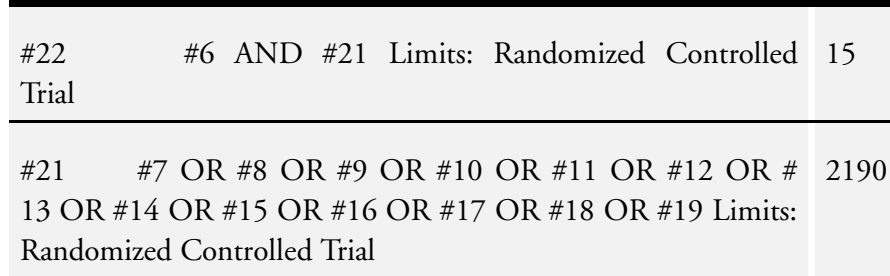


(Continued)

\begin{tabular}{|c|c|c|}
\hline \multicolumn{2}{|r|}{$\begin{array}{l}\# 20 \text { \#7 OR \#8 OR \#9 OR \#10 OR \#11 OR \#12 OR \#13 OR } \\
\# 14 \text { OR \#15 OR \#16 OR \#17 OR \#18 OR \#19 }\end{array}$} & 15315 \\
\hline \#19 & Search acupoint injection $[\mathrm{tw}]$ & 81 \\
\hline \#18 & trigger point $[\mathrm{tw}]$ & 520 \\
\hline \#17 & dry needling [tw] & 84 \\
\hline \#16 & scalp acupuncture $[\mathrm{tw}]$ & 110 \\
\hline$\# 15$ & auricular acupuncture $[\mathrm{tw}]$ & 201 \\
\hline$\# 14$ & ear acupuncture $[\mathrm{MeSH}]$ & 197 \\
\hline$\# 13$ & electro-acupuncture $[\mathrm{tw}]$ & 518 \\
\hline \#12 & electro-acupuncture $\{\mathrm{MeSH}]$ & 0 \\
\hline$\# 11$ & electroacupuncture $[\mathrm{MeSH}]$ & 2024 \\
\hline$\# 10$ & body acupuncture $[\mathrm{tw}]$ & 102 \\
\hline$\# 9$ & acupuncture point $[\mathrm{MeSH}]$ & 2962 \\
\hline$\# 8$ & acupuncture therapy $[\mathrm{MeSH}]$ & 14011 \\
\hline$\# 7$ & acupuncture $[\mathrm{MeSH}]$ & 14710 \\
\hline $\begin{array}{l}\# 6 \\
\text { Trial }\end{array}$ & \#1 OR \#2 OR \#3 OR \#4 Limits: Randomized Controlled & 397 \\
\hline$\# 5$ & $\# 1$ OR \#2 OR \#3 OR \#4 & 6753 \\
\hline$\# 4$ & chronic widespread pain $[\mathrm{tw}]$ & 305 \\
\hline$\# 3$ & fibromyalgia syndrome [tw] & 1146 \\
\hline$\# 2$ & fibromyal* $[\mathrm{tw}]$ & 6664 \\
\hline$\# 1$ & fibromyalgia [MeSH] & 5234 \\
\hline$\# 3$ & fibromyalgia syndrome $[\mathrm{tw}]$ & 1146 \\
\hline \#2 & fibromyal ${ }^{*}[\mathrm{tw}]$ & 6664 \\
\hline
\end{tabular}




\section{Appendix 3. EMBASE search strategy and results}

(Updated search on 17 January 2012)

$\# 1$

(Acupuncture and fibromyalgia).mp. [mp=title, abstract, subject headings,

heading word, drug trade name, original title, device manufacturer, drug

manufacturer, device trade name, keyword]

limit 1 to $\mathrm{yr}=$ “2010 - 2012”

\section{Appendix 4. CINAHL search strategy and results}

(Via EBSCOhost updated search on 17 January 2012 then 28 March 2012)

TX Acupuncture AND TX Fibromyalgia

2

limit 1 to yr="Jan 2010 - Dec 2011”
535

Appendix 5. Chongqing Weipu (VIP) search strategy and results

\begin{tabular}{|c|c|}
\hline Search terms & $\begin{array}{l}\text { Vip } \\
1989-2010 \\
(\mathrm{tw}=\text { 任意字段; } \quad \text { abstract=文摘 })\end{array}$ \\
\hline \# 1 纤维肌痛 $[\mathrm{tw}]$ & 273 \\
\hline \# 2 纤维肌痛 [abstract] & 212 \\
\hline ＃３纤维肌痛综合征 [tw] & 201 \\
\hline \# 4 纤维肌痛综合征 [abstract] & 158 \\
\hline
\end{tabular}

Copyright $\odot 2013$ The Cochrane Collaboration. Published by John Wiley \& Sons, Ltd. 
(Continued)

\begin{tabular}{|c|c|}
\hline \# 5 RCT [abstract ] & 6443 \\
\hline \# 6 随机对照试验 [abstract] & 3504 \\
\hline \# 7临床科研 $[\mathrm{tw}]$ & 2620 \\
\hline \# 8临床观察 [tw] & 172039 \\
\hline \# 9 随机对照 [tw] & 15101 \\
\hline \# 10 针刺 $[\mathrm{tw}]$ & 42588 \\
\hline \# 11 针炎 $[\mathrm{tw}]$ & 67944 \\
\hline \# 12 电针 $[\mathrm{tw}]$ & 10444 \\
\hline \# 13 耳针 $[\mathrm{tw}]$ & 1118 \\
\hline \# 14 头针 $[\mathrm{tw}]$ & 1952 \\
\hline$\# 15$ 水针 $[\mathrm{tw}]$ & 2281 \\
\hline $\begin{array}{l}\# 1 \text { or } \# 2 \text { or } \# 3 \text { or } \# 4 A N D \# 5 \text { or } \# 6 \text { or } \# 7 \text { or } \# 8 \text { or } \# 9 \text { AND \# } \\
10 \text { or } \# 11 \text { or } \# 12 \text { or } \# 13 \text { or } \# 14 \text { or } \# 15\end{array}$ & 35 \\
\hline
\end{tabular}

\section{Appendix 6. Wanfang search strategy and results}

\begin{tabular}{|c|c|}
\hline Search terms & $\begin{array}{l}\text { Wangfang } \\
\text { Inception to } 2010\end{array}$ \\
\hline \# 1 纤维肌痛 [tw] & 4109 \\
\hline \# 2 纤维肌痛 [abstract] & 3090 \\
\hline \# 3 纤维肌痛综合征 [tw] & 131 \\
\hline ＃４纤维肌痛综合征[abstract] & 146 \\
\hline \# 5 RCT [abstract ] & 4977 \\
\hline \# 6 随机对照试验 [abstract] & 3686 \\
\hline \# 7临床科研 $[\mathrm{tw}]$ & 1696 \\
\hline \# 8临床观察 $[\mathrm{tw}]$ & 141525 \\
\hline
\end{tabular}




\begin{tabular}{|c|c|}
\hline \# 9 随机对照 $[\mathrm{tw}]$ & 16801 \\
\hline \# 10 针制 $[\mathrm{tw}]$ & 37681 \\
\hline \# 11 针负 $[\mathrm{tw}]$ & 52905 \\
\hline \# 12 电针 $[\mathrm{tw}]$ & 10225 \\
\hline \# 13 耳针 [tw] & 807 \\
\hline \# 14 头针 $[\mathrm{tw}]$ & 1731 \\
\hline$\# 15$ 水针 $[\mathrm{tw}]$ & 1481 \\
\hline $\begin{array}{l}\# 1 \text { or } \# 2 \text { or } \# 3 \text { or } \# 4 \text { AND } \# 5 \text { or } \# 6 \text { or } \# 7 \text { or } \# 8 \text { or } \# 9 \text { AND \# } \\
10 \text { or } \# 11 \text { or } \# 12 \text { or } \# 13 \text { or } \# 14 \text { or } \# 15\end{array}$ & 28 \\
\hline
\end{tabular}

\section{Appendix 7. National Research Register search strategy and results}

(Updated search on 21 January 2012)

You searched for fibromyalgia AND acupuncture

"There are no results."

\section{Appendix 8. HSRProj search strategy and results}

(Updated search on 21 January 2012)

((fibromyalgia AND acupuncture) AND (randomised controlled trial))

0 result found

\section{Appendix 9. Current Contents search strategy and results}

(via Web of Science updated search on 17 January 2012 then 28 March 2012)

1

Topic $=($ Acupuncture $)$ AND Topic $=($ fibromyalgia $)$

99

Databases=ABES, SBS, CM, LS, PCES, ECT, AH, BC, EC Times-

pan $=$ All Years

Lemmatization $=O n$

2

Topic $=($ Acupuncture $)$ AND Topic $=($ fibromyalgia $)$

Refined by: Publication Years $=(2010$ OR 2011$)$

Databases=ABES, SBS, CM, LS, PCES, ECT, AH, BC, EC Times-

pan $=$ All Years

Lemmatization $=O n$

Copyright $\odot 2013$ The Cochrane Collaboration. Published by John Wiley \& Sons, Ltd. 


\section{Appendix I0. STRICTA}

Detailed information of acupuncture treatment (modified STRICTA items)

\begin{tabular}{|c|c|c|c|c|c|c|c|c|c|}
\hline Study ID & $\begin{array}{l}\text { Assefi } \\
2005\end{array}$ & $\begin{array}{l}\text { Deluze } \\
1992\end{array}$ & $\begin{array}{l}\text { Guo } \\
2005\end{array}$ & $\begin{array}{l}\text { Harris } \\
2005\end{array}$ & $\begin{array}{l}\text { Harris } \\
2008\end{array}$ & $\begin{array}{l}\text { Harris } \\
2009\end{array}$ & $\begin{array}{l}\text { Itoh } \\
2010\end{array}$ & $\begin{array}{l}\text { Martin } \\
2006\end{array}$ & $\begin{array}{l}\text { Targino } \\
2008\end{array}$ \\
\hline $\begin{array}{l}\text { Acupunc- } \\
\text { ture style }\end{array}$ & $\begin{array}{l}\text { Manual } \\
\text { acupunc- } \\
\text { ture with } \\
\text { formula } \\
\text { points }\end{array}$ & $\begin{array}{l}\text { Electro- } \\
\text { acupunc- } \\
\text { ture with } \\
\text { formula } \\
\text { points }\end{array}$ & $\begin{array}{l}\text { Manual } \\
\text { point-to- } \\
\text { point } \\
\text { threading } \\
\text { acupunc- } \\
\text { ture with } \\
\text { formula } \\
\text { points }\end{array}$ & $\begin{array}{l}\text { Manual } \\
\text { acupunc- } \\
\text { ture with } \\
\text { formula } \\
\text { points }\end{array}$ & $\begin{array}{l}\text { Manual } \\
\text { acupunc- } \\
\text { ture with } \\
\text { formula } \\
\text { points }\end{array}$ & $\begin{array}{l}\text { Manual } \\
\text { acupunc- } \\
\text { ture with } \\
\text { formula } \\
\text { points }\end{array}$ & $\begin{array}{l}\text { Electro- } \\
\text { acupunc- } \\
\text { ture } \\
\text { plus trigger } \\
\text { point } \\
\text { acupunc- } \\
\text { ture }\end{array}$ & $\begin{array}{l}\text { Electro- } \\
\text { acupunc- } \\
\text { ture plus } \\
\text { formula } \\
\text { CM }\end{array}$ & $\begin{array}{l}\text { Manual } \\
\text { acupunc- } \\
\text { ture with } \\
\text { formula } \\
\text { points }\end{array}$ \\
\hline $\begin{array}{l}\text { Rationale } \\
\text { for treat- } \\
\text { ment in- } \\
\text { cluding } 3 \\
\text { items: CM } \\
\text { diagnosis/ } \\
\text { point } \\
\text { selection/ } \\
\text { trial proto- } \\
\text { col }\end{array}$ & $\begin{array}{l}\text { NR/NR/ } \\
\text { NR }\end{array}$ & $\begin{array}{l}\text { NR/ } \\
\text { individu- } \\
\text { alised with } \\
2 \text { main } \\
\text { points/ } \\
\text { points se- } \\
\text { lection and } \\
\text { EA } \\
\text { was based } \\
\text { on journal } \\
\text { articles }\end{array}$ & $\begin{array}{l}\text { NR/NR/ } \\
\text { NR Only } \\
\text { mentioned } \\
\text { "Standard } \\
\text { treatment" }\end{array}$ & $\begin{array}{l}\text { NR/points } \\
\text { selected } \\
\text { based } \\
\text { on "ability } \\
\text { to reduce } \\
\text { symptoms } \\
\text { of FM"/ } \\
\text { NR }\end{array}$ & $\begin{array}{l}\mathrm{NR} / \text { points } \\
\text { selected } \\
\text { based } \\
\text { on Harris } \\
2005 \\
\text { study/NR }\end{array}$ & $\begin{array}{l}\mathrm{NR} / \text { points } \\
\text { selected } \\
\text { based } \\
\text { on Harris } \\
2005 \\
\text { study/NR }\end{array}$ & $\begin{array}{l}\text { NR/NR/ } \\
\text { NR }\end{array}$ & $\begin{array}{l}\mathrm{NR} / \text { points } \\
\text { standard- } \\
\text { ised } \\
\text { formula } \\
\text { "strong } \\
\text { regulatory } \\
\text { points"/ } \\
\mathrm{NR}\end{array}$ & $\begin{array}{l}\mathrm{NR} / \text { points } \\
\text { selected } \\
\text { based on } \\
\text { "Classi- } \\
\text { cal"/based } \\
\text { on clinical } \\
\text { experience }\end{array}$ \\
\hline $\begin{array}{l}\text { Sources to } \\
\text { justify ra- } \\
\text { tionale }\end{array}$ & $\begin{array}{l}\text { Clinical } \\
\text { experience } \\
\text { and discus- } \\
\text { sion with } 3 \\
\text { other } \\
\text { acupunc- } \\
\text { turists }\end{array}$ & $\begin{array}{l}\text { Text- } \\
\text { book and } \\
\text { journal ar- } \\
\text { ticles } \\
\text { (refer- } \\
\text { enced) }\end{array}$ & $\begin{array}{l}\text { Classic lit- } \\
\text { erature and } \\
\text { research } \\
\text { papers } \\
\text { (not refer- } \\
\text { enced) }\end{array}$ & $\begin{array}{l}\text { Textbook } \\
\text { (refer- } \\
\text { enced) } \\
\text { However } \\
\text { this text } \\
\text { does not } \\
\text { specifically } \\
\text { state those } \\
\text { points } \\
\text { are for fi- } \\
\text { bromyal- } \\
\text { gia }\end{array}$ & $\begin{array}{l}\text { Referenced } \\
\text { to Harris } \\
2005 \\
\text { paper }\end{array}$ & $\begin{array}{l}\text { Referenced } \\
\text { to Harris } \\
2005 \\
\text { paper }\end{array}$ & NR & NR & $\begin{array}{l}\text { Referenced } \\
\text { to WHO } \\
\text { standard } \\
\text { nomencla- } \\
\text { ture, } \\
\text { but the ref- } \\
\text { erence } \\
\text { is not re- } \\
\text { lated to fi- } \\
\text { bromyal- } \\
\text { gia diagno- } \\
\text { sis or treat- } \\
\text { ment }\end{array}$ \\
\hline $\begin{array}{l}\text { Points } \\
\text { used in real } \\
\text { acupunc- } \\
\text { ture treat- } \\
\text { ment }\end{array}$ & $\begin{array}{l}\text { Alternat- } \\
\text { ing } \\
\text { between } \\
\text { LI11, SP9, } \\
\text { CV12, } \\
\text { ST25, } \\
\text { KI7, TE5, } \\
\text { Ex-HN-3 } \\
\text { (Yin Tang) }\end{array}$ & $\begin{array}{l}\text { LI4, ST36 } \\
\text { plus up to } \\
6 \text { other } \\
\text { points } \\
\text { which were } \\
\text { not } \\
\text { reported }\end{array}$ & $\begin{array}{l}\text { Along GV } \\
\text { meridian } \\
\text { and the } 2 \\
\text { lines of the } \\
\text { Bladder } \\
\text { meridian. } \\
\text { Exact start } \\
\text { and finish } \\
\text { points not }\end{array}$ & $\begin{array}{l}\text { Unilateral } \\
\text { Left LI11, } \\
\text { ST36 SP6, } \\
\text { GB34 } \\
\text { Right LI4, } \\
\text { LR3, plus } \\
\text { GV20 and } \\
\text { ear point } \\
\text { shenmen }\end{array}$ & $\begin{array}{l}\text { As } \\
\text { per Harris } \\
2005 \text { study }\end{array}$ & $\begin{array}{l}\text { As } \\
\text { per Harris } \\
2005 \text { study }\end{array}$ & $\begin{array}{l}\text { Points se- } \\
\text { lected us- } \\
\text { ing trigger } \\
\text { point ther- } \\
\text { apy but } \\
\text { does } \\
\text { state apart } \\
\text { from mus- } \\
\text { cle groups }\end{array}$ & $\begin{array}{l}\text { Bilateral } \\
\text { LI4, ST36, } \\
\text { LR2, SP6 } \\
\text { PC6, HT7 } \\
\text { plus } 3 \text { cer- } \\
\text { vical and } 4 \\
\text { lumber ax- } \\
\text { ial on BL }\end{array}$ & $\begin{array}{l}\text { Ex-HN-3 } \\
\text { (Yin Tang) } \\
\text { LR3, } \\
\text { LI4, PC6, } \\
\text { GB34 and } \\
\text { SP6 }\end{array}$ \\
\hline
\end{tabular}




\begin{tabular}{|c|c|c|c|c|c|c|c|c|c|}
\hline & $\begin{array}{l}\text { and } \\
\text { KI7, } \\
\text { BL17, } \\
\text { BL18, } \\
\text { BL20, } \\
\text { BL22, } \\
\text { BL43, } \\
\text { BL44 }\end{array}$ & & reported & & & & $\begin{array}{l}\text { what they } \\
\text { were }\end{array}$ & $\begin{array}{l}\text { channel } \\
\text { but did not } \\
\text { state actual } \\
\text { points }\end{array}$ & \\
\hline $\begin{array}{l}\text { Uni/ } \\
\text { bilateral }\end{array}$ & $\begin{array}{l}\text { Not } \\
\text { reported } \\
\text { clearly }\end{array}$ & Bilateral & NR & As above & $\begin{array}{l}\text { As } \\
\text { per Harris } \\
2005 \text { study } \\
\text { [MCIT1] }\end{array}$ & $\begin{array}{l}\text { As } \\
\text { per Harris } \\
2005 \text { study }\end{array}$ & Bilateral & As above & $\begin{array}{l}\text { All bilat- } \\
\text { eral except } \\
\text { for Ex- } \\
\mathrm{HN}-3\end{array}$ \\
\hline $\begin{array}{l}\text { Number of } \\
\text { needles in- } \\
\text { serted }\end{array}$ & $7-14$ & $\begin{array}{l}10 \text { (study } \\
\text { used } \\
5 \text { pairs of } \\
\text { electrodes) }\end{array}$ & NR & 9 & 9 & 9 & $\begin{array}{l}\text { Real } 10 \text { for } \\
10 \text { wks } \\
\text { Non- } \\
\text { acupunc- } \\
\text { ture nil for } \\
5 \text { wks, then } \\
5 \text { after wk } \\
5\end{array}$ & $\begin{array}{l}18 \text { first } 3 \text { tx } \\
20 \text { final } 3 \\
\text { tx }\end{array}$ & 11 \\
\hline $\begin{array}{l}\text { Depths of } \\
\text { insertion }\end{array}$ & $\begin{array}{l}\text { "Standard } \\
\text { depth" } \\
\text { (refer- } \\
\text { enced to a } \\
\text { textbook) }\end{array}$ & $\begin{array}{l}\text { Real: } 10 \text { to } \\
25 \mathrm{~mm} \\
\text { Control: } 3 \\
\text { to } 4 \mathrm{~mm}\end{array}$ & $\begin{array}{l}\text { Subcuta- } \\
\text { neous }\end{array}$ & $\begin{array}{l}\text { All } \\
\text { groups had } \\
\text { the same } \\
\text { depth, } \\
20 \text { to } 30 \\
\mathrm{~mm}\end{array}$ & $\begin{array}{l}\text { Real } 20 \\
\text { mm } \\
\text { Sham non- } \\
\text { penetrat- } \\
\text { ing }\end{array}$ & $\begin{array}{l}\text { Real } 20 \\
\mathrm{~mm} \\
\text { Sham non- } \\
\text { penetrat- } \\
\text { ing }\end{array}$ & $\begin{array}{l}\text { EA } 5 \text { to } 20 \\
\text { mm } \\
\text { Trigger } \\
\text { point } 10 \text { to } \\
20 \mathrm{~mm}\end{array}$ & $\begin{array}{l}\text { NR, } \\
\text { But figure } \\
\text { shows } \\
\text { needling } \\
\text { into mus- } \\
\text { cles }\end{array}$ & $\begin{array}{l}10 \text { to } 30 \\
\mathrm{~mm} \text {, per- } \\
\text { pendic- } \\
\text { ular inser- } \\
\text { tion for all } \\
\text { points ex- } \\
\text { cept when } \\
\text { needling } \\
\text { Ex-HN-3 } \\
\text { which was } \\
\text { obliquely } \\
\text { inserted }\end{array}$ \\
\hline $\begin{array}{l}\text { Responses } \\
\text { elicited }\end{array}$ & $\begin{array}{l}\text { "Stimula- } \\
\text { tion" } \\
\text { stated but } \\
\text { not clear if } \\
\text { deqi was } \\
\text { elicited }\end{array}$ & $\begin{array}{l}\text { Real: deqi } \\
\text { elicited } \\
\text { Control: } \\
\text { no deqi }\end{array}$ & NR & $\begin{array}{l}\text { Deqi was } \\
\text { elicited in } \\
2 \text { out of the } \\
4 \text { arms }\end{array}$ & $\begin{array}{l}\text { Real: deqi } \\
\text { was } \\
\text { elicited on } \\
\text { all points } \\
\text { below the } \\
\text { neck }\end{array}$ & $\begin{array}{l}\text { Real: deqi } \\
\text { was } \\
\text { elicited on } \\
\text { all points } \\
\text { below the } \\
\text { neck }\end{array}$ & $\begin{array}{l}\text { Deqi on } \\
\text { both EA } \\
\text { and trigger } \\
\text { point }\end{array}$ & $\begin{array}{l}\text { No deqi in- } \\
\text { tended }\end{array}$ & $\begin{array}{l}\text { Deqi was } \\
\text { elicited }\end{array}$ \\
\hline $\begin{array}{l}\text { Type of } \\
\text { nee- } \\
\text { dle stimu- } \\
\text { lation }\end{array}$ & Manual & $\begin{array}{l}\text { Electrical } \\
\text { real: visible } \\
\text { muscle } \\
\text { twitch } \\
10 \mathrm{~mA} \mathrm{-} \\
\text { contin- } \\
\text { uous Con- }\end{array}$ & $\begin{array}{l}\text { Point-to- } \\
\text { point } \\
\text { threading, } \\
\text { even } \\
\text { movement }\end{array}$ & $\begin{array}{l}\text { Lifting and } \\
\text { thrusting } \\
\text { with even } \\
\text { rotation } \\
(12 \text { rota- } \\
\text { tions at }\end{array}$ & Manual & Manual & $\begin{array}{l}\text { EA visible } \\
\text { mus- } \\
\text { cle twitch, } \\
4 \mathrm{~Hz} \text {, rect- } \\
\text { angu- } \\
\text { lar bipha- }\end{array}$ & $\begin{array}{l}\text { Electrical, } \\
2 \mathrm{~Hz}, \mathrm{LI} 4 \\
\text { and ST36 } \\
\text { plus } 10 \mathrm{~Hz} \\
\text { (alter- } \\
\text { nating cer- }\end{array}$ & Manual \\
\hline
\end{tabular}




\begin{tabular}{|c|c|c|c|c|c|c|c|c|c|}
\hline & & $\begin{array}{l}\text { trol: } \\
\text { no muscle } \\
\text { twitch set- } \\
\text { ting simi- } \\
\text { lar to real } \\
\text { group but } \\
\text { weaker }\end{array}$ & & $\begin{array}{l}180^{\circ} \text { clock } \\
\text { and anti- } \\
\text { clockwise }\end{array}$ & & & $\begin{array}{l}\text { sic top } \\
\text { Trigger } \\
\text { point } \\
\text { "Sparrow } \\
\text { pecking" }\end{array}$ & $\begin{array}{l}\text { vical and } \\
\text { lumber BL } \\
\text { channel) }\end{array}$ & \\
\hline $\begin{array}{l}\text { Needle re- } \\
\text { tention } \\
\text { time }\end{array}$ & 30 minutes & NR & 30 minutes & 20 minutes & 25 minutes & 25 minutes & $\begin{array}{l}\text { EA } 15 \\
\text { minutes } \\
\text { Trigger } \\
\text { point } 15 \\
\text { minutes }\end{array}$ & 20 minutes & 20 minutes \\
\hline $\begin{array}{l}\text { Needle } \\
\text { size/ } \\
\text { length/ } \\
\text { type/ man- } \\
\text { ufacturer }\end{array}$ & $\begin{array}{l}\text { NR/34 } \\
\text { to } 40 \mathrm{~mm} / \\
\text { Chinese, } \\
\text { Japanese, } \\
\text { Korean/ } \\
\text { NR }\end{array}$ & $\begin{array}{l}0.3 \mathrm{~mm} / \\
25 \mathrm{~mm} / \\
\text { Stainless } \\
\text { steel/NR }\end{array}$ & $\begin{array}{l}0.35 \mathrm{~mm} / \\
40 \mathrm{~mm} / \\
\mathrm{NR} / \mathrm{NR}\end{array}$ & $\begin{array}{l}25 \mathrm{~mm} / 38 \\
\mathrm{~mm} / \\
\text { Stainless } \\
\text { steel/ } \\
\text { HBW } \\
\text { Supply Inc }\end{array}$ & $\begin{array}{l}25 \mathrm{~mm} / 50 \\
\mathrm{~mm} / \\
\text { Stainless } \\
\text { steel/ } \\
\text { Seirin }\end{array}$ & $\begin{array}{l}\text { NR/NR/ } \\
\text { NR/NR }\end{array}$ & $\begin{array}{l}0.2 \mathrm{~mm} / \\
40 \mathrm{~mm} / \\
\text { stainless } \\
\text { steel/Seirin }\end{array}$ & $\begin{array}{l}\text { NR/NR/ } \\
\text { NR/NR } \\
\text { EA unit } \\
\text { IC-1107+, } \\
\text { ITO, } \\
\text { Japan }\end{array}$ & $\begin{array}{l}25 \mathrm{~mm} / 40 \\
\mathrm{~mm} / \mathrm{NR} / \\
\mathrm{NR}\end{array}$ \\
\hline $\begin{array}{l}\text { Number of } \\
\text { treatment } \\
\text { sessions }\end{array}$ & $\begin{array}{l}24 \text { over } 12 \\
\text { wks }\end{array}$ & $\begin{array}{l}6 \text { over } 3 \\
\text { wks }\end{array}$ & $\begin{array}{l}28 \text { over } 30 \\
\text { days with } \\
14 \text { for each } \\
\text { course; } \\
\text { a rest of } 2 \\
\text { days } \\
\text { in between } \\
\text { courses }\end{array}$ & $\begin{array}{l}18 \text { over } 13 \\
\text { wks } \\
\text { Forced- } \\
\text { titration } \\
\text { paradigm } \\
\text { (1 tx wkly } \\
3 \text { wks), ( } \\
\text { tx wkly } 3 \\
\text { wks) } \\
\text {, } 3 \text { tx wkly } \\
3 \text { wks) } \\
2 \\
\text { washout } \\
\text { be- } \\
\text { tween each } \\
\text { tx group }\end{array}$ & $\begin{array}{l}9 \text { over } 4 \\
\text { wks }\end{array}$ & $\begin{array}{l}9 \text { over } 4 \\
\text { wks }\end{array}$ & $\begin{array}{l}10 \text { over } 10 \\
\text { wks } \\
\text { (this was a } \\
\text { cross-over } \\
\text { study after } \\
5 \text { weeks. } \\
1 \text { st } 5 \text { weeks } \\
\text { tx was only } \\
\text { on } 1 \text { arm } \\
\text { with the } \\
\text { other non- } \\
\text { acupunc- } \\
\text { ture) }\end{array}$ & $\begin{array}{l}6 \text { over } 3 \\
\text { wks }\end{array}$ & $\begin{array}{l}20 \text { over } 3 \\
\text { months }\end{array}$ \\
\hline $\begin{array}{l}\text { Fre- } \\
\text { quency of } \\
\text { treatments }\end{array}$ & $\begin{array}{l}\text { Twice } \\
\text { weekly }\end{array}$ & $\begin{array}{l}\text { Twice } \\
\text { weekly }\end{array}$ & Daily & As above & $\begin{array}{l}\text { Twice to } 3 \\
\text { times } \\
\text { weekly }\end{array}$ & $\begin{array}{l}\text { Twice } \\
\text { weekly }\end{array}$ & Weekly & $\begin{array}{l}\text { Every } 2 \text { to } \\
4 \text { days over } \\
2 \text { to } 3 \text { wks }\end{array}$ & $\begin{array}{l}\text { Twice } \\
\text { weekly }\end{array}$ \\
\hline $\begin{array}{l}\text { Practi- } \\
\text { tioner } \\
\text { back- } \\
\text { ground: } \\
\text { training, } \\
\text { clinical ex- }\end{array}$ & $\begin{array}{l}8 \text { acupunc- } \\
\text { turists } \\
\text { received } \\
\text { standard- } \\
\text { ised train- }\end{array}$ & NR & $\begin{array}{l}\text { Au- } \\
\text { thors from } \\
\text { Chinese } \\
\text { medicine } \\
\text { university }\end{array}$ & $\begin{array}{l}\text { Point loca- } \\
\text { tion deter- } \\
\text { mined by } \\
2 \text { licensed } \\
\text { acupunc- }\end{array}$ & $\begin{array}{l}1 \text { acupunc- } \\
\text { turist } \\
\text { trained at } \\
\text { the Mary- } \\
\text { land Insti- }\end{array}$ & NR & $\begin{array}{l}1 \text { acupunc- } \\
\text { turist, } \\
4 \quad \text { yrs } \\
\text { acupunc- } \\
\text { ture train- }\end{array}$ & $\begin{array}{l}2 \text { acupunc- } \\
\text { turists but } \\
\text { no details } \\
\text { reported }\end{array}$ & $\begin{array}{l}1 \text { acupunc- } \\
\text { turist } \\
\text { (physician) } \\
\text { with 5 } \\
\text { years clini- }\end{array}$ \\
\hline
\end{tabular}




\begin{tabular}{|c|c|c|c|c|c|}
\hline $\begin{array}{l}\text { perience, } \\
\text { exper- } \\
\text { tise in spe- } \\
\text { cific condi- } \\
\text { tion }\end{array}$ & $\begin{array}{l}\text { ing in the } \\
\text { different } \\
\text { styles for } \\
\text { the } \\
\text { study. Trial } \\
\text { acupunc- } \\
\text { tur- } \\
\text { ist had } 15 \\
\text { years ex- } \\
\text { perience in } \\
\text { treating fi- } \\
\text { bromyal- } \\
\text { gia } \\
\text { patients }\end{array}$ & $\begin{array}{l}\text { turists with } \\
12 \text { yrs ex- } \\
\text { perience in } \\
\text { fibromyal- } \\
\text { gia and } \\
17 \text { yrs ex- } \\
\text { perience in } \\
\text { acupunc- } \\
\text { ture. } 95 \% \\
\text { of } \\
\text { tx done by } \\
1 \text { acupunc- } \\
\text { turist }\end{array}$ & $\begin{array}{l}\text { tute of Tra- } \\
\text { ditional } \\
\text { Chinese } \\
\text { Medicine } \\
\text { with } 6 \\
\text { years clini- } \\
\text { cal } \\
\text { acupunc- } \\
\text { ture expe- } \\
\text { rience. No } \\
\text { expertise } \\
\text { in a spe- } \\
\text { cific condi- } \\
\text { tion }\end{array}$ & $\begin{array}{l}\text { ing and } \\
\text { clinical ex- } \\
\text { perience of } \\
3 \text { or } 10 \mathrm{yrs}\end{array}$ & $\begin{array}{l}\text { cal experi- } \\
\text { ence }\end{array}$ \\
\hline
\end{tabular}

Abbreviations used: CM: Chinese medicine; EA: electro-acupuncture; mm: millimetre; NR: not reported; tx: treatments; wk = weeks: wkly = weekly; yrs: years

\section{WHAT'S NEW}

Last assessed as up-to-date: 15 January 2012.

\begin{tabular}{|c|c|c|}
\hline Date & Event & Description \\
\hline 10 May 2008 & Amended & CMSG ID A019-R \\
\hline 13 April 2008 & Amended & Converted to new review format. \\
\hline
\end{tabular}

\section{CONTRIBUTIONSOFAUTHORS}

John C Deare (JD)

- Topic conception, protocol development and revision

- Systematic review study selection, methodology, adequacy of treatment, data extraction, data analysis and interpretation of findings

- Co-author of the review

Zhen Zheng (ZZ)

- Topic conception, protocol development review and revision

- Systematic review study selection, methodology, adequacy of treatment, data extraction, data analysis and interpretation of findings

- Co-author of the review 
Charlie C Xue (CX)

- Topic conception and methodological aspects

- Protocol review

- Systematic review dispute resolution for study selection, adequacy of treatment, and revision and review of final interpretations of findings

Jian Ping Liu (JPL)

- Topic conception, methodological perspectives, data analysis

- Protocol revision and review

- Systematic review dispute resolution for methodological quality and final proof

Jingsheng Shang (JSS)

- Topic conception

- Protocol review proof

- Systematic review final proof

Sean W Scott (SS)

- Protocol review proof

- Systematic review data extraction

- Systematic review final proof

Geoff Littlejohn (GL)

- Protocol review proof

- Systematic review final proof

\section{DECLARATIONSOF INTEREST}

The authors plan to conduct an acupuncture clinical trial on fibromyalgia in the future. No other potential conflicts of interest have been noted. 


\section{SOURCES OF SUPPORT}

\section{Internal sources}

- Traditional \& Complementary Medicine Program, Health Innovations Research Institute, Discipline of Chinese Medicine, School of Health Sciences, RMIT University, Australia.

- Compmed Health Institute, Southport, Australia.

- Centre for Clinical Research and Evaluation, Beijing University of Chinese Medicine, Beijing, China.

\section{External sources}

- National Center for Complementary and Alternative Medicine (NCCAM, Number R24 AT001293), National Institutes of Health (NIH), USA.

JD was supported by NCCAM grant. The contents of this systematic review are solely the responsibility of the authors and do not necessarily represent the official views of the NCCAM or the NIH.

- National Health and Medical Research Council (NHMRC Project Grants 555411 and 555414), Australia.

$\mathrm{ZZ}$ and CCLX were partially supported by the NHMRC grants. The contents of this systematic review are solely the responsibility of the authors and do not necessarily represent the official views of the NHMRC.

\section{DIFFERENCES BETWEEN PROTOCOLANDREVIEW}

We updated the background and changed the order of importance of the main and minor outcomes, based on findings from reviews. In accordance with new Cochrane Collaboration guidelines, we have included in the review 'Risk of bias' and replaced the planned 'Clinical relevance tables' with the 'Summary of findings' tables. We did not conduct the searches of ACULARS, AcuBriefs, SIGLE or AMED as they could either not be accessed via RMIT University, it was a pay for service or the content of the databases was covered by our other searches. 